\title{
The nature and efficacy of culturally-adapted psychosocial interventions in schizophrenia: a systematic review and meta-analysis
}

DOI:

10.1017/S0033291717002264

\section{Document Version}

Accepted author manuscript

Link to publication record in Manchester Research Explorer

Citation for published version (APA):

Degnan, A., Baker, S., Edge, D., Nottidge, W., Noke, M., Husain, N., Rathod, S., \& Drake, R. (2017). The nature and efficacy of culturally-adapted psychosocial interventions in schizophrenia: a systematic review and metaanalysis. Psychological Medicine. https://doi.org/10.1017/S0033291717002264

\section{Published in:}

Psychological Medicine

\section{Citing this paper}

Please note that where the full-text provided on Manchester Research Explorer is the Author Accepted Manuscript or Proof version this may differ from the final Published version. If citing, it is advised that you check and use the publisher's definitive version.

\section{General rights}

Copyright and moral rights for the publications made accessible in the Research Explorer are retained by the authors and/or other copyright owners and it is a condition of accessing publications that users recognise and abide by the legal requirements associated with these rights.

\section{Takedown policy}

If you believe that this document breaches copyright please refer to the University of Manchester's Takedown Procedures [http://man.ac.uk/04Y6Bo] or contact uml.scholarlycommunications@manchester.ac.uk providing relevant details, so we can investigate your claim.

\section{OPEN ACCESS}




\section{Psychological Medicine \\ The nature and efficacy of culturally-adapted psychosocial interventions for schizophrenia: a systematic review and meta-analysis \\ --Manuscript Draft--}

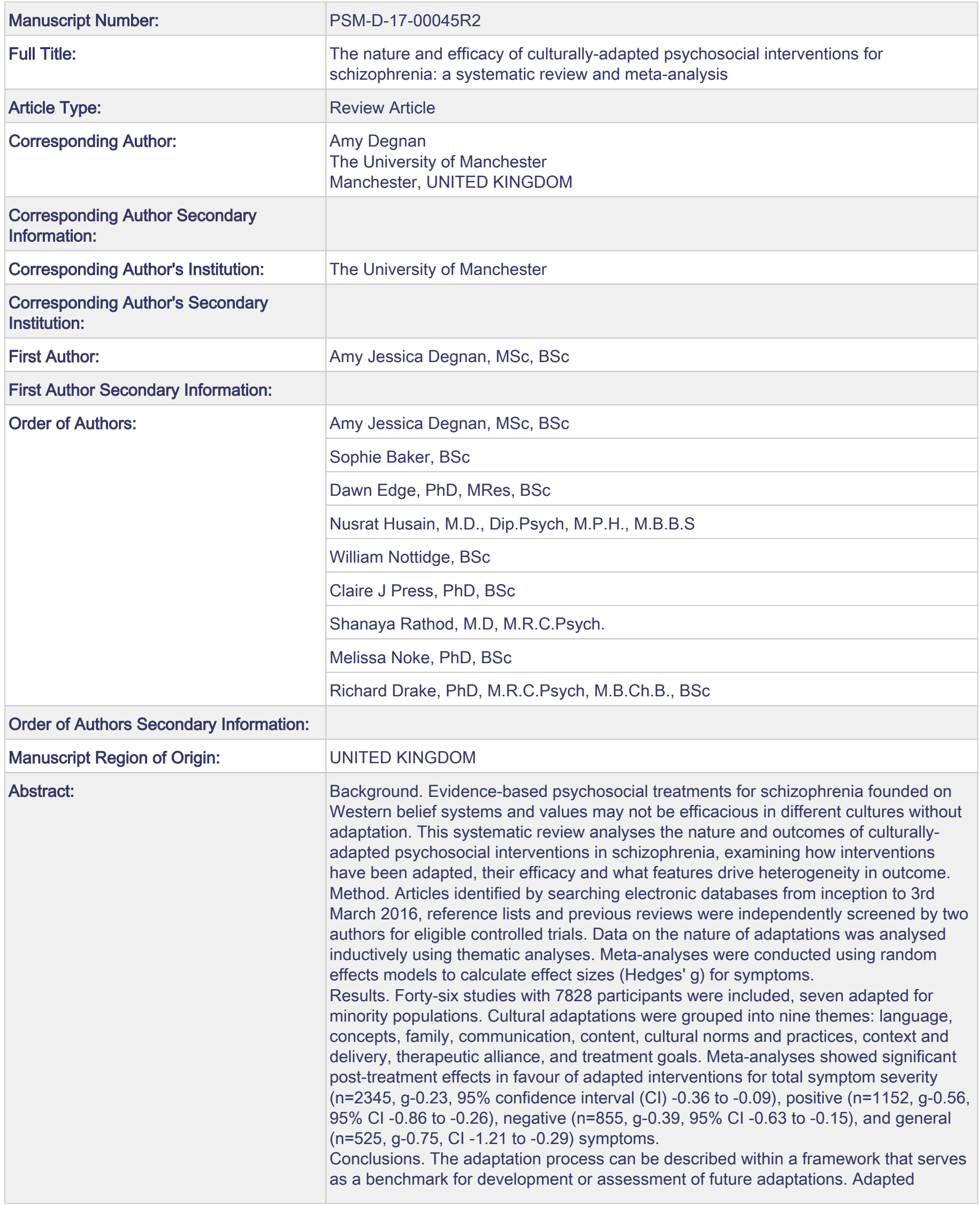


interventions were more efficacious than usual treatment in proportion to the degree of adaptation. Both context and design influenced efficacy. Investigating whether adaptation improves efficacy, most importantly amongst ethnic minorities, requires more comparisons against unadapted interventions. 


\section{The nature and efficacy of culturally-adapted psychosocial interventions for} schizophrenia: a systematic review and meta-analysis

\section{Authors and affiliations}

*Amy Degnan, Division of Psychology \& Mental Health; Faculty of Biology, Medicine \& Health; The University of Manchester; amy.degnan@manchester.ac.uk

Sophie Baker, Division of Psychology \& Mental Health; Faculty of Biology, Medicine \& Health; The University of Manchester; sophie.baker@manchester.ac.uk

Dawn Edge, Division of Psychology \& Mental Health; Faculty of Biology, Medicine \& Health; The University of Manchester; dawn.edge@manchester.ac.uk

Nusrat Husain, Division of Psychology \& Mental Health; Faculty of Biology, Medicine \& Health Sciences; The University of Manchester; Lancashire Care NHS Foundation Trust, Preston; Sceptre Point, Sceptre Way, Walton Summit, Preston, PR5 6AW; nusrat.husain@manchester.ac.uk

William Nottidge, care of Division of Psychology \& Mental Health; Faculty of Biology, Medicine \& Health; The University of Manchester

Claire J Press, care of Division of Psychology \& Mental Health; Faculty of Biology, Medicine \& Health; The University of Manchester

Shanaya Rathod, MD, MRCPsych. Consultant Psychiatrist and Director of Research, Southern Health NHS Foundation Trust, Clinical Trials Facility, Tom Rudd Unit, Moorgreen Hospital; West End, Southampton, SO30 3JB; shanaya.rathod@southernhealth.nhs.uk Melissa Noke; care of Division of Psychology \& Mental Health; Faculty of Biology, Medicine \& Health; The University of Manchester

Richard Drake, Division of Psychology \& Mental Health; Faculty of Biology, Medicine \& Health; The University of Manchester; Greater Manchester Mental Health NHS Foundation Trust, Park House, Delaunays Road, Manchester M8 5RB; richard.drake@manchester.ac.uk

\section{${ }^{*}$ Corresponding author:}

Room 3.306 Jean McFarlane Building, Division of Psychology \& Mental Health, Faculty of Biology, Medicine \& Health, The University of Manchester, M13 9PL 


\section{Abstract}

Background. Evidence-based psychosocial treatments for schizophrenia founded on Western belief systems and values may not be efficacious in different cultures without adaptation. This systematic review analyses the nature and outcomes of culturally-adapted psychosocial interventions in schizophrenia, examining how interventions have been adapted, their efficacy and what features drive heterogeneity in outcome.

Method. Articles identified by searching electronic databases from inception to $3^{\text {rd }}$ March 2016, reference lists and previous reviews were independently screened by two authors for eligible controlled trials. Data on the nature of adaptations was analysed inductively using thematic analyses. Meta-analyses were conducted using random effects models to calculate effect sizes (Hedges' g) for symptoms.

Results. Forty-six studies with 7828 participants were included, seven adapted for minority populations. Cultural adaptations were grouped into nine themes: language, concepts and illness models, family, communication, content, cultural norms and practices, context and delivery, therapeutic alliance, and treatment goals. Meta-analyses showed significant posttreatment effects in favour of adapted interventions for total symptom severity $(n=2345, \mathrm{~g}$ $0.23,95 \%$ confidence interval $(\mathrm{Cl})-0.36$ to -0.09$)$, positive $(n=1152, g-0.56,95 \% \mathrm{Cl}-0.86$ to $-0.26)$, negative $(n=855, g-0.39,95 \% \mathrm{Cl}-0.63$ to -0.15$)$, and general $(n=525, g-0.75, C l-1.21$ to -0.29$)$ symptoms.

Conclusions. The adaptation process can be described within a framework that serves as a benchmark for development or assessment of future adaptations. Culturally adapted interventions were more efficacious than usual treatment in proportion to the degree of adaptation. There is insufficient evidence to show that adapted interventions are better than non-adapted interventions. Features of context, intervention and design influenced efficacy. Investigating whether adaptation improves efficacy, most importantly amongst ethnic minorities, requires better designed trials with comparisons against unadapted interventions.

\section{Key words}

Cultural-adaptation, schizophrenia, psychosis, ethnic minorities, psychosocial intervention 


\section{Introduction}

Migrant and ethnic minority populations have, approximately, a three-fold increased incidence of schizophrenia (Cantor-Graae, 2007, Cantor-Graae and Selten, 2005). In the United Kingdom (UK), elevated rates of schizophrenia have been reported for Black and Minority Ethnic (BME) populations when compared to the majority White British population, with the highest rates for Black Caribbean, followed by Black African and then Asian groups (Fearon et al., 2006). Ethnic disparities exist in treatment access, experiences and outcomes of schizophrenia, with disadvantages consistently reported for minority populations in Western countries (i.e. countries derived from and influenced by European cultures). UK research and policies have highlighted inequalities for ethnic minorities, including poorer engagement with services and professionals, more coercive care pathways, compulsory hospital admissions and involvement in the criminal justice system, higher doses of medication and inferior access to psychological therapies (Bhui et al., 2003, Department of Health, 2005, Keating et al., 2002, The Sainsbury Centre for Mental Health, 2006).

Current guidelines in the UK and United States of America (USA) (Dixon et al., 2010, National Institute for Health and Care Excellence, 2014) recommend cognitive behavioural therapy (CBT) and family intervention (FI) for schizophrenia based on meta-analytic evidence of effectiveness (Pharoah et al., 2010, Wykes et al., 2008). However, it is unclear whether the effectiveness of these interventions generalises across ethnic groups since ethnic minorities remain underrepresented in clinical trials of most psychological interventions (Brown et al., 2014, Hussain-Gambles et al., 2004, Waheed et al., 2015). It has been suggested that outcomes of psychosocial interventions are poorer for ethnic minorities than Caucasians (Bhugra et al., 1997). This is perhaps not surprising given that most psychosocial interventions have been developed in the West (i.e. Europe or the USA) and are underpinned by Western cultural values. Culture has been defined as '...the set of distinctive spiritual, material, intellectual and emotional features of society or a social group... it encompasses, in addition to art and literature, lifestyles, ways of living together, value systems, traditions and beliefs.' (United Nations Educational, Scientific and Cultural Organisation (UNESCO)) (Torres, 2002). There is evidence that people are more likely to seek help and engage with psychiatric and psychological treatment when their cultural beliefs and explanatory models are considered (Bhui and Bhugra, 2004, Carter et al., 2016, Rathod et al., 2010). Therefore, to improve acceptability and effectiveness of treatment, there has been growing interest in adapting interventions in schizophrenia to be congruent with the cultural contexts and values of minority populations (e.g. Edge et al., 2016, Rathod et al., 2013). Adaptation and evaluation of such interventions is also necessary to allow 
psychosocial treatment to be evidenced in majority populations in low and middle income countries' (LMIC) (Feigin, 2016).

Systematic reviews of cultural-adaptations of psychosocial interventions for mental illness (Benish et al., 2011, Chowdhary et al., 2014, Griner and Smith, 2006, Huey and Polo, 2008, Smith et al., 2011) find adapted interventions' mean effect sizes for primary outcomes (0.410.72) comparable to non-adapted interventions in Western populations (e.g. 0.35-0.44; Wykes et al., 2008). However, most systematic reviews have included diagnostically and ethnically mixed samples and not attempted to disentangle how far these factors shape interventions' effects. Few have analysed the nature of cultural adaptations systematically to provide an empirically derived framework or model of adaptation. Without a framework to describe adaptation it is difficult to examine what type or degree of adaptation was efficacious.

Chowdhary et al. (2014) conducted a systematic review of 20 controlled trials of culturally adapted psychological treatments for depression, including CBT, interpersonal therapy, psychoeducation, problem-solving therapy and dynamic oriented therapy. They used a framework developed by Bernal and Sáez-Santiago (2006) to describe adaptations, which were mostly within the language, therapist and context dimensions. They reported effect size (SMD) - 0.72 for depressive symptoms but noted that the small number of studies, incompleteness of data and significant heterogeneity in context, interventions and study design prevented comparison of the degree or types of adaptations across different interventions.

Bhui et al. (2015) reviewed 21 studies of various designs to identify adaptations in a wide range of interventions designed to improve therapeutic communications between BME patients and clinicians in psychiatric services. Thematic analyses classified adaptations somewhat broadly using Tseng's (2001) framework, which includes ethnic matching, changes to structure and content, technical delivery or structure of therapy, working with social systems, and facilitating empowerment and engagement. Culturally-adapted psychotherapies, ethnographic and motivational assessment were found to be effective and preferred by patients and carers; and high quality trials' efficacy for outcomes including symptoms and medication adherence were $d=0.18-0.75$.

It is therefore clear that evidence-based psychosocial interventions for schizophrenia spectrum diagnoses, originally developed in the West are an important element of therapy that require adaptation for both minority ethnic groups in Western countries; and for majority ethnic groups in non-Western countries. However, it is not clear to what extent these contrasting types of adaptation are successful, or even whether different types of adaptation 
and target ethnic groups are comparable. One obstacle to understanding adaptations' importance in psychosocial interventions for schizophrenia is that none of the existing frameworks describing cultural adaptations (Barrera and Castro, 2006, Bernal and Sáez-Santiago, 2006, Hwang, 2009, Tseng, 2001) focus on interventions specific to this area. The review therefore had two aims: 1) to analyse inductively the nature of culturaladaptations to psychosocial interventions and develop a framework describing the adaptations identified across controlled trials in schizophrenia; and 2) to assess the efficacy of adapted psychosocial interventions for schizophrenia spectrum diagnoses and examine the effect of differences between interventions, samples and other context and design features on outcome

\section{Methods}

The review followed Preferred Reporting Items for Systematic Reviews and Meta-analyses (PRISMA) guidelines (Moher et al., 2009).

\section{Search strategy and study selection}

On $3^{\text {rd }}$ March 2016 the lead author (AD) conducted an electronic database search of Ovid MEDLINE, EMBASE, PsycINFO and Web of Science. Databases were searched from inception. See A1 online supplementary material for search strategy. Two co-authors (AD and SB) independently screened the articles for eligibility using the following criteria:

\section{Inclusion criteria}

- Trials of any design evaluating a culturally adapted evidence-based psychosocial intervention (i.e. psychotherapies or interventions that address psychological and social factors to improve psychological and social functioning).

- Adaptations are made to the format, delivery or content of an existing Western intervention (i.e. influenced by European culture, including Europe, USA, Canada and Australia) to meet the cultural needs of a specific ethnic group or subculture (i.e. minority culture within a larger dominant culture).Trials evaluate interventions adapted for a minority ethnic population in a Western country or any non-Western population.

- Participants 18 years+ with a diagnosis of schizophrenia (DSMIV or ICD-10 F20-29: schizophreniform disorder, schizoaffective disorder, delusional disorder or psychosis not otherwise specified).

- At least one validated patient outcome measure to provide information on clinical effectiveness

- Peer-reviewed published articles available in English 


\section{Exclusion criteria}

- Adaptations are generalised across multiple ethnic groups (and no findings available per group, despite author contact)

- Interventions without specific adaptations for culture; including assessment of an existing intervention in a different subculture or ethnic group without adaptation, direct translation only, or adapting for some other characteristic such as age or location (e.g. rural vs. urban)

- A novel intervention developed specifically for a particular subculture or ethnic group without adaptation of an existing evidence-based intervention (derived from a Western model)

- Non-evaluative studies (e.g. literature reviews, qualitative studies, case studies)

Full text papers of potentially relevant articles were accessed and screened by two reviewers (AD \& SB). Authors were contacted to request English versions of non-English citations and those that could not be accessed. Reference lists of full text articles and systematic literature reviews were screened to identify any additional papers not picked up in the search. Key experts were contacted with full reference lists to identify missing studies. All uncertainties or disagreements relating to eligibility of articles were resolved via discussion with RD.

\section{Data extraction}

Descriptive characteristics of eligible studies were recorded in a data extraction spreadsheet. All adaptations described in the papers were extracted and summarised. Where there was limited information, corresponding authors were contacted and adaptations were extracted from any material they provided such as written summaries, protocol and treatment manuals. Sample size, means and standard deviations for symptom scores for adapted intervention(s) and control(s)) at each time point (baseline and follow-up(s)) were recorded. Authors were contacted for unreported data to calculate effect sizes.

\section{Analysis of cultural-adaptation}

Our aim was to review the current literature on cultural adaptations in schizophrenia and provide a synthesis that was grounded in empirical work and evidence. Thematic analysis (Braun and Clarke, 2006) was applied inductively to the extracted and summarised data on adaptations to generate themes and subthemes emerging from the data. This therefore reflected current application in the field, rather than deductively applying an existing model or framework to the data. We used Braun and Clarke's six phased approach: 1) data familiarisation; 2) generating codes (identifying adaptations in most basic form); 3) searching 
for themes (identifying common areas of adaptation repeated across studies and combine into themes and subthemes); 4) reviewing themes; and 5) defining and naming themes.

\section{Analysis of outcomes}

Meta-analyses were conducted to examine the effects of culturally-adapted interventions on symptoms in schizophrenia. We focused on symptoms as this was the most commonly reported outcome, and assessed using reliable, validated and comparable scales. Only RCTs were included in the meta-analyses as they provide the most robust evidence of effectiveness (Concato et al., 2000).

Meta-analyses were performed using Review Manager (version 5.3) software. Effect sizes were calculated using Hedge's (adjusted) $g$, which consists of the difference between the means of the adapted intervention versus the control group divided by the pooled standard deviation (corrected for sample size) and then further weighted for sample size (Hedges and Olkin, 2014). 95\% confidence intervals (Cl) are presented.

Cochrane's risk of bias tool (Higgins et al., 2011) was used to assess the quality of RCTs by rating the level of bias (unclear, low, high) across six domains: sequence generation, allocation concealment, blinding of outcome assessors, incomplete outcome data, selective outcome reporting, and other sources of bias. Four RAs independently assessed all the studies and the lead author (AD) made final decisions on discrepant ratings. Publication bias was explored by examination of funnel plots (Higgins and Green, 2008) and there was evidence of slight asymmetry for positive symptoms (Egger et al., 1997). See online supplementary for risk of bias assessments (A2) and funnel plots (A3).

Heterogeneity of effects was assessed using $R$ tests (Higgins and Thompson, 2002) and explored using sensitivity and subgroup analyses to see if any outliers, pre-treatment differences, or intervention or study characteristics biased the results (see Table 1 for list of variables in subgroup analyses). Given the expected and observed variation across studies, random effects models were applied as they provide conservative estimates adjusted for observed heterogeneity (Brockwell and Gordon, 2001, Kontopantelis and Reeves, 2010).

\section{Results}

\section{Study characteristics}

46 papers comprising 43 individual studies with 7828 participants were included; 31 simple RCTs, 12 cluster RCTs, one block RCT and two non-randomised pilot trials. Sample sizes ranged from six to 3082 with a mean of 182 . See Figure 1 for the PRISMA flow chart and online supplementary for Table A4 of descriptive characteristics of studies. 


\section{Intervention characteristics}

Interventions were delivered in 13 different countries. The majority $(74 \%, n=34)$ were conducted in Asia (25 in China, two in each of Pakistan, Taiwan, India and one in each of Iran, Saudi Arabia and Malaysia). Nine studies (20\%) were conducted in America (six in the USA; two in Mexico; and one in Brazil) and one study (2\%) was conducted in each of Europe (Italy), Australia and Africa (Egypt). Most interventions $(85 \%, n=39)$ were adapted for a majority population with only seven studies (15\%) adapted for a minority population.

Around half of the studies $(54 \%, n=25)$ were family interventions $(\mathrm{FI})$ consisting of a psychoeducational or mutual support component. Fls varied across the studies in terms of their components and the evidence-based models they were adapted from. Twenty two studies (88\%) included group FI sessions. In 12 (48\%) studies, all of the sessions were designed for the family members and patients to attend together, in five (20\%) patients attended at least part of the intervention $(25-86 \%$ of the sessions), and in eight (32\%) only family members were invited. Ten studies (22\%) evaluated some form of cognitive therapy, consisting of three social cognitive skills training, one social cognitive remediation therapy, three cognitive behavioural therapies (CBT), two meta-cognitive training (MCT), and one integrated psychological therapy (IPT). Family members attended two of the CBT interventions; the remaining eight cognitive interventions were for patients only. Three studies (7\%) were combined interventions comprising components adapted from multiple Western therapy manuals and theoretical frameworks. One of these was a combined psychosocial intervention that included family therapy sessions. The other two were symptom coping programmes for patients only. Five studies (11\%) assessed social skills training (SST). Family members attended four of the SST interventions. Of the remaining interventions, two (4\%) were illness management and recovery (IMR) programmes and one (2\%) comprised a mindfulness based psycho-education programme, both for patients only.

Over half of the interventions $(59 \%, n=27)$ were delivered in a clinical setting. Six were delivered in community settings and four delivered sessions in both clinical and community settings. Nine studies did not report intervention setting. Duration of interventions ranged from three weeks (Lin et al., 2013) to two years (Carrà et al., 2006; Xiong et al., 1994; Zhang \& Heqin, 1993; Zhang et al., 1998), with a mean of eight months. The majority of the interventions were led by mental health professionals $(80 \%, n=37)$; five of these (all assessing group FIs) were co-facilitated by a family member participating in the study. Five studies did not specify therapist training.

Characteristics of the interventions are described in supplementary Table A5. 


\section{Cultural adaptations}

A total of nine themes emerged from the data on adaptations. Details of the themes describing cultural-adaptations, with examples from the reviewed studies, are described in online supplementary (Table A6).

\section{Language}

Language adaptations were reported in all 46 studies: translating the original intervention into the national language (e.g. Bradley et al., 2006, Koolaee and Etemadi, 2009, Kumar et al., 2010, Xiang et al., 1994), including local colloquialisms and idioms to improve cultural relevance and acceptability (Gohar et al., 2013, Habib et al., 2015, Naeem et al., 2015, So et al., 2015), and/or exchanging jargon for more culturally-appropriate words (e.g. replacing 'module' with 'treatment areas'; Valencia et al. 2010; Naeem et al., 2015, So et al., 2015).

\section{Concepts and illness models}

Most interventions $(78 \%, n=36)$ incorporated culturally appropriate presentations of concepts, with consideration of culture-specific belief systems, enhanced mental health stigma and low levels of education. This included working with alternatives to the 'biopsychosocial' model, including the attribution of mental illness to spiritual or supernatural agents (Kopelowicz et al., 2012, Razali et al., 2000), predestination and fate (Koolaee and Etemadi, 2009), and an imbalance of yin and yang forces (Chan et al., 2009). Some studies reported the inclusion of spiritual factors in formulations and discussion of locally held beliefs in psychoeducation (e.g. Habib et al., 2015, Naeem et al., 2015). Stigma was addressed by sharing personal stories and recovery narratives for normalisation, and holding group forums for participants to discuss concerns (Chien, 2008, Chien and Chan, 2004, Guo et al., 2010, Lin et al., 2013). Due to varied understanding and experience of mental illness and low education levels in certain cultural contexts, adaptations were made to alter the complexity and amount of psychoeducation or therapy material provided to make it more manageable for patients and families (Kung et al., 2012, Patterson et al., 2005, Zimmer et al., 2007).

\section{Family}

Most individual interventions $(76 \%, n=35)$ were adapted to acknowledge the pivotal role of the family in patient's care and recovery, as well as culturally distinct family structures and processes. This included efforts to encourage families' active and continued involvement throughout the intervention (Carrà et al., 2007, Habib et al., 2015); e.g. offering additional sessions or informal home visits for family members and maintaining contact after treatment (Bradley et al., 2006, Kung et al., 2012, Shin and Lukens, 2002, Xiong et al., 1994). Modifications to accommodate more interdependent family structures and higher value being 
put on familial responsibility than individualism (e.g. Chien, 2008, Li and Arthur, 2005) included involving family members in decision-making, assessing the needs of the family as a whole, and for example emphasising how medication adherence would benefit the family unit rather than the individual (Kopelowicz et al., 2012, Mausbach et al., 2008, Patterson et al., 2005). Further considerations included sensitivity to culture-specific roles and expectations, e.g. expecting younger members not to question their elders (Chien and Chan, 2004, Koolaee and Etemadi, 2009, Valencia et al., 2010).

\section{Communication}

Twenty two (48\%) studies reported adaptations to integrate culturally-specific ways of communicating and learning. This included culturally-appropriate methods for dealing with conflict and problem solving, e.g. preference for reparative action rather than discussion in Chinese cultures (Chien, 2008, Chien and Chan, 2004, 2013, Chien and Lee, 2010, Chien et al., 2008)) and replacing concepts of assertiveness and expression of individual needs in the West with mutual respect and avoidance of confrontation in more family dominant cultures (Habib et al., 2015, Kung et al., 2012, Patterson et al., 2005). Considerations were made in relation to the disclosure of private information, due to the reluctance to openly discuss family matters (Chien and Chan, 2013) or irrelevance of confidentiality in close-knit families (Valencia et al., 2010, Valencia et al., 2007). Culturally appropriate teaching methods were also used; for example, encouraging collaboration and active participation in more passive cultures (Kopelowicz et al., 2003, Patterson et al., 2005), and using practical rehearsals and visual aids (Chien and Lee, 2010, Shin and Lukens, 2002).

\section{Content}

Twenty interventions (43\%) made modifications to content. Content was added or removed from original manuals (Gohar et al., 2013, Razali et al., 2000, So et al., 2015, Valencia et al., 2010, Zimmer et al., 2007) to improve cultural relevance (e.g. So et al. (2015) removed references to a conspiracy theory about a Western celebrity in meta-cognitive therapy for Chinese people) or feasibility (e.g. Valencia et al. (2010) omitted video-assisted modelling social skills training from the Mexican version due to limited technology).

\section{Cultural norms}

Adaptations were made to incorporate culture specific norms and practices in 31 (67\%) studies. Interventions incorporated spiritual or religious practices and coping methods such as traditional healers, religious texts and prayer (Bradley et al., 2006, Habib et al., 2015, Wahass and Kent, 1997). Culturally relevant activities and scenarios were also integrated, e.g. Karaoke, Tai Chi and Mahjong (Mann and Chong, 2004); Baduanjin relaxation exercises (Kung et al., 2012); and traditional folk stories/characters in role plays, recordings and videos 
(Habib et al., 2015, Lak et al., 2010, Wahass and Kent, 1997). Recognising the social structures of certain cultures, additional efforts were made to incorporate community support through peer leaders, group meetings/workshops/seminars, and social gatherings outside of therapy (Chien and Lee, 2010, Kung et al., 2012, Ran et al., 2003, Xiang et al., 1994).

\section{Context}

Almost half of the studies $(48 \%, n=22)$ reported adaptations to facilitate feasibility in specific cultural contexts. These involved addressing cultural norms or organisational barriers due to lack of commitment, funding or resources (Kung et al., 2012, Li and Arthur, 2005, Lin et al., 2013). For example, delivering interventions at accessible locations where there are sufficient resources (e.g. Carrà et al., 2007, Li and Arthur, 2005), offering flexibility in scheduling sessions (e.g. Guo et al., 2010, Habib et al., 2015); changing the duration of treatment (e.g. Kung et al., 2012, Lin et al., 2013); seeing patients and family together or separately (e.g. Carrà et al., 2007, Kulhara et al., 2009, Kung et al., 2012); and the use of group versus individual interventions (e.g. Chien and Chan, 2004, Chien and Thompson, 2013).

\section{Therapeutic alliance}

Adaptations to improve therapeutic alliance were present in $28 \%(n=13)$ of studies. These included matching therapists and clients for ethnicity and other characteristics such as age, gender or language to enhance acceptability (e.g. Bradley et al., 2006, Koolaee and Etemadi, 2009). A few studies reported training or supervising therapists to improve cultural competency (Kopelowicz et al., 2003, Kopelowicz et al., 2012). Other studies reported modifications to build rapport, trust and engagement; e.g. therapists engaging in small talk and warm up activities before the intervention (Kopelowicz et al., 2003, Valencia et al., 2010, Wang et al., 2013), and presenting appropriate forms of self-disclosure from their own lives to facilitate a more personalised therapeutic relationship (Valencia et al., 2010).

\section{Treatment goals}

These were modified in thirteen studies (28\%) to develop formulations that were realistic and congruent with cultural values. These involved developing shared goals to meet the needs of the family unit and managing expectations of different family members; for example, the tendency to expect immediate and practical help from close relatives in Chinese cultures (Chien and Chan, 2004, Chien et al., 2006).

\section{Efficacy}


All but four RCTs (Carrà et al., 2007, Chien, 2008, Chien et al., 2004, Wang et al., 2013) evaluated the efficacy of culturally-adapted interventions against symptoms using validated scales, most often the BPRS or the PANSS (Table A4).

31 of the 40 RCTs examining symptoms were two-armed trials. Six (Chien \& Chan, 2004; Chien \& Chan, 2013; Chien, Chan \& Thompson, 2006; Koolaee \& Etemadi, 2010; Kopelowicz et al., 2012; Lak et al., 2010) comprised two adapted interventions and a third comparison group. Two of these reported results from the same trial (Chien \& Chan, 2004; Chien et al., 2006). Three trials (Chien \& Thompson, 2013; Mausbach et al., 2008; Ran et al., 2003) included one adapted intervention and two comparison groups.

Two studies (Gohar et al., 2013; Mausbach et al., 2008) compared the adapted intervention to the non-adapted version of the same intervention in a particular context, the gold standard test of whether modifying for culture is more effective than not. Both studies found no significant between-group differences in efficacy. One (Mausbach et al., 2008) was excluded from the meta-analysis because it included the same participants as an earlier study (Patterson et al., 2005) and had inadequate random allocation methods. Ten RCTs (Mann \& Chong, 2004; Ran et al., 2003; Razali et al., 2000; Wahass \& Kent, 1997; Weng et al., 2003; Xian et al., 1994; Xiong et al., 1994; Zhang \& Hequin, 1993; Zhang et al., 1998; Zimmer et al., 2007) were excluded because sufficient data to allow calculation of $g$ were neither reported nor could be obtained from the authors.

Therefore 29 studies were included in the meta-analyses on total, positive, negative and general symptoms. For forest plots, see Figure 2 for total symptoms and A7 supplementary for positive, negative and general symptoms. See Table 1 for summary statistics for subgroup analyses for total symptoms and A8 supplementary for positive, negative and general symptoms.

\section{Post treatment effects}

\section{Total symptoms}

For the 19 RCTs ( $n=2345)$ examining total symptom scores post-treatment (0-3 months), effect of the adapted intervention significantly exceeded that of control ( $g:-0.23$; Cl-0.36,$0.09 ; p<.001)$, with moderate heterogeneity $\left(X^{2}=34.72\right.$, df18, $\left.p<.001, l^{2} 48 \%\right)$ (Figure 2). To explore what study parameters might moderate heterogeneity or efficacy, we divided these studies into various subgroups (Table 1). Distinguishing between studies of minorities in the West and adaptation to ethnic majorities in LMIC reduced overall heterogeneity (see $I^{2}$ ), efficacy being significantly greater in studies of minorities ( $\mathrm{Cl}$ for mean difference in g:-0.67, $0.01 ;$ p0.044). Interventions attended by service users and family members were trend- 
significantly more efficacious than those attended by service users only $(\mathrm{Cl}$ for mean difference in g:-0.66, 0.01; p0.057). Distinguishing interventions by clinical setting (versus community) reduced heterogeneity (see $l^{2}$ ) without there being a significant difference in efficacy between groups. Other potential moderating variables neither significantly reduced $P^{2}$ in all subgroups nor identified groups with significantly different efficacy.

\section{Positive symptoms}

16 studies ( $n=1152$ ) examined the effects of the adapted intervention on positive symptoms post-intervention (0-3 months). There was significant efficacy ( $g:-0.56, \mathrm{Cl}-0.86,-0.26$, $p<.001)$, but substantial heterogeneity $\left(X^{2}=86.99\right.$, df15, $\left.p<.001, P^{2} 83 \%\right)$. No potential moderators substantially reduced heterogeneity $\left(I^{2}\right)$ across all subgroups but cluster randomised trials had significantly poorer efficacy than others $(\mathrm{Cl}$ for mean difference in $\mathrm{g}: 0.25,01.12 ; \mathrm{p} 0.036)$; and Chinese studies had significantly poorer efficacy ( $\mathrm{Cl}$ for mean difference in $\mathrm{g}:-1.50,-0.47 ; \mathrm{p} 0.018)$.

\section{Negative symptoms}

12 studies $(n=855)$ reported post-treatment effects on negative symptoms ( $g$ :-0.39, Cl-0.63,$0.15)$, with moderate heterogeneity $\left(x^{2} 30.79\right.$, df11, $\left.p<.001, I^{2} 64 \%\right)$. Studies using standard care as a control were more efficacious than those using an active control ( $p 0.076)$ or enhanced care (p0.042) and this reduced heterogeneity $\left(I^{2}\right)$ in all groups. Dividing studies into family versus individual interventions reduced heterogeneity across all groups (difference in efficacy, p0.096).

\section{General symptoms}

Eight studies $(n=525)$ examining PANSS general symptoms post-treatment demonstrated significant effects of the adapted intervention versus standard care (g:-0.75, Cl-1.21,-0.29) and high heterogeneity $\left(X^{2} 40.99, \mathrm{df} 7, p<.001, l^{2} 83 \%\right)$. Otherwise, distinguishing studies with more or less attrition than $15 \%$ reduced overall heterogeneity slightly ( $270 \%$ in each group). There were no significant differences between subgroups in efficacy.

\section{Sensitivity analyses}

Further to examine the effect of methodology on outcomes we performed a range of other planned sensitivity analyses.

\section{Individual studies}

For each symptomatic outcome, studies were removed individually to identify any difference to mean weighted effect size or heterogeneity. Most showed negligible effects. However, one positive outlier (Habib et al., 2015) reduced the effect size and heterogeneity when 
removed from analyses of positive ( $g:-0.44 \&{ }^{2} 77 \%$ ), negative ( $g:-0.34 \&{ }^{2} 60 \%$ ) and general symptoms ( $g:-0.55 \&{ }^{2} 63 \%$ ), but findings remained significant in favour of the adapted intervention ( $p$ <.001). Removing two studies (Bradley et al., 2006; Gohar et al., 2013) with highest risk of bias (i.e. open trial; 'as treated' analyses) increased efficacy for negative symptoms (g:-0.45 and 0.46) and positive symptoms (g:-0.61, Gohar et al., 2013). Excluding one study (So et al., 2015) with pre-treatment differences and high attrition (>40\%) increased the effect size for general symptoms ( $g:-0.87)$ and positive symptoms ( $g:-0.60)$.

Follow up assessments

Separate analyses were conducted at multiple follow-up points; each analysis included fewer studies $(n \leq 6)$ than the post-treatment analyses. For total symptoms, the aggregated effect was significant at 3, 6, 12 and 18-24 months follow-up (g0.18 to 1.00), with significant and large heterogeneity at 18-24 months (1279). Meta-analyses for positive symptoms showed significant effects at 12 months ( $g:-0.33$; $\mathrm{Cl}-0.54,-0.11)$ but not at 3,6 or $15-18$ months. Negative symptoms at 6 months follow-up showed significant improvement compared to controls ( $g:-0.27 ; \mathrm{Cl}-0.51,-0.04$ ) based on data from two studies. There was no significant heterogeneity for positive and negative symptoms at follow-up. Only one study assessed general symptoms at 6 months (Kopelowicz et al., 2003) ( $\mathrm{n}=84$, g:-0.09, Cl-0.52, 0.34, $p=0.67)$.

\section{Three armed trials}

For three-armed trials with two adapted interventions and one control (Chien \& Chan, 2004; Chien \& Chan, 2013; Chien, Chan \& Thompson, 2006; Koolaee \& Etemadi, 2010; Kopelowicz et al., 2012; Lak et al., 2010), all effects remained significant in favour of the adapted intervention when the above analyses were repeated exchanging the adapted intervention of interest with the second adapted intervention. One trial (Chien \& Thompson, 2013) had one adapted intervention and two comparator groups but there was no difference in effect depending on whether data from the active (psychoeducation) or standard care control was included.

Degree of adaptation

There was a significant correlation between greater number of adaptations and better total symptom efficacy $(r-0.49, \mathrm{p} 0.034)$. Regression confirmed that $\mathrm{g}$ improved 0.1 for each adaptation (B-0.097, $\beta=-.487$ ). A9 supplementary details the adaptations for the reviewed studies.

\section{Discussion}


The present review and meta-analysis is the first to synthesise current evidence on the nature and effectiveness of culturally-adapted psychosocial interventions in schizophrenia. Thematic analyses of cultural-adaptations reported in each of the reviewed studies produced a framework that serves as a benchmark for future adaptations. It also provided an indication of the degree of adaptation for any given intervention, notwithstanding the caveat that not all adaptations are necessary or possible in different contexts.

We found considerable agreement regarding what constitutes cultural-adaptation. All studies reported adaptations to language. The majority made adaptations in the domains of concepts and illness models, family and cultural norms and practices: considering explanatory models of illness, incorporating spiritual/religious activities, and acknowledging culture-specific familial structures (e.g. interdependent and hierarchical). Other common adaptations were to recognise different forms of communicating, learning and problemsolving, removal of culturally-irrelevant content, and changing aspects of delivery to recognise contextual barriers. Studies also made modifications to improve engagement and therapeutic alliance and develop shared treatment goals. As previously reported in relation to adapted treatments for depression (Chowdhary et al., 2014), authors reported changes to delivery to improve acceptability and feasibility in specific cultural contexts, rather than the core components of the interventions, thus maintaining their underlying theoretical models.

Our meta-analysis demonstrated significant effects in favour of the adapted intervention at post-treatment and follow-up. Post-treatment effect sizes for symptomatic outcomes (total symptoms $g-0.23$, positive $g$-0.46, negative $g$-0.39) were similar to those for non-adapted interventions (e.g. CBT SMD positive $=0.37$, negative $=0.44$; Wykes et al. 2008).

Since methodological quality can influence effect sizes (Tarrier and Wykes, 2004), with large differences in results from meta-analyses including RCTs versus those with observational or quasi-experimental designs (Pfammatter et al., 2006), we restricted inclusion to studies with RCT designs, homogeneous ethnic groups and 100\% schizophrenia samples. This may explain the more modest efficacy against total symptoms than in previous reviews of adapted interventions which included non-randomised trials and mixed ethnic and diagnostic samples (e.g. Chowdhary et al., 2014; Griner \& Smith, 2006). Nonetheless, methodology still varied considerably, with differences in allocation (cluster/block, standard), control condition (standard care, active), and quality (e.g. 'intention to treat' analysis, blinding, attrition). Only two trials (Gohar et al., 2010; Mausbach et al., 2008) compared adapted and non-adapted interventions, the gold standard test of whether adaptation improves efficacy. Neither found significant treatment differences for psychopathology; Mausbach et al. (2008) was excluded as it comprised the same sample as an earlier study (Patterson et al., 2005) but had poor 
randomisation methods and Gohar et al. (2013), though included, was an open trial. Moreover, few studies used active control conditions. We can therefore only conclude that adapting interventions for culture is more effective than usual care. Sensitivity analyses provided limited evidence that removal of individual trials with high risk of bias (i.e. pretreatment differences, open trial and 'as treated' analyses) increased the effect size. Future studies using better designs, preferably three-armed RCTs, must be recommended to evaluate whether adapted interventions are more efficacious than standard care and their non-adapted equivalents.

To make a meta-analysis meaningful, one must assume that different interventions for a particular diagnosis have common elements that produce common efficacy in analogous populations. Where this is not the case, heterogeneity occurs and must be examined to reveal what processes influence outcome. The results showed that different types of interventions had generally similar efficacy, which suggests that each have common elements that may well be important to outcome even in diverse populations. However, there was substantial heterogeneity for each outcome, unsurprising given the diverse contexts and interventions (Higgins \& Green, 2008). Subgroup analyses showed that features of context (i.e. Chinese or not; ethnic minority/Western or majority/ non-Western population), intervention (i.e. individual or family participation) and study design (i.e. randomisation method; control group) moderated outcome but were difficult to disentangle and there was limited evidence of a consistent pattern across symptomatic outcomes. The majority of studies were adapted for a majority population and were in Asian countries; with around half conducted in China. There was some evidence that studies of ethnic minorities in Western countries or outside China were more efficacious than studies of ethnic majority populations in non-Western countries or those in China. However, due to the small number of studies looking at minority populations, we were unable to examine efficacy of adaptations by ethnic minority group. Subgroup analyses also suggested that interventions attended by family members were more efficacious. Findings relating to differences in specific intervention models (e.g. CBT versus MCT) are inconclusive; though we attempted to examine intervention types in the subgroup analyses, categories were broad due to the limited number of studies available. Future trials should continue to test adapted interventions for specific ethnic or cultural populations with schizophrenia diagnoses to build on the current evidence base.

Limitations of this review include variation across studies in the quality of reporting of methodology. All papers reported some level of adaptation, but often poorly. Some authors did not respond to requests for additional information which may have led to an incomplete 
picture of adaptation. Additionally, our empirically derived framework is based on adapted interventions reported in clinical trials. However, although beyond the scope of this review, it is worth noting that we reviewed our thematic framework against adaptations reported in non-evaluative studies that were generated from an initial broad search of the literature (with no restriction on design). Its application worked well, with no additional themes of adaptation identified. Some studies with incomplete statistical data were excluded from the metaanalyses, despite several attempts to contact authors. Poor reporting of methods in reviewed papers proved to be problematic for analysing overall risk of bias which meant we focused on three aspects of quality with the most variation (i.e. attrition, 'intention to treat' analysis, pre-treatment differences) in our heterogeneity analyses. We did not include unpublished studies but funnel plots reassured us of no more than minimal positive bias for positive symptoms due to selective reporting. We focused on symptomatic outcomes as this was the most common reported and consistently measured outcome. However, this outcome may not be the most appropriate or meaningful outcome for all psychosocial interventions. A limitation arises from the original studies that did not provide evidence as to how often symptomatic improvement was clinically significant. It is therefore unclear how criteria for such clinical significance translate across different cultures. Future studies should aim to measure the effects of adaptation on other patient outcomes, such as functioning and relapse. Another avenue for future research would be to consider caregiver outcomes and more qualitative data relating to the acceptability of culturally-adapted interventions to patients, carers and healthcare professionals.

Preliminary findings suggest that the greater the degree of cultural-adaptation the more efficacious the intervention. Although greater adaptation might correlate with unmeasured features of studies, such as quality of interventions or therapists, our framework may provide a useful heuristic model to guide clinicians and researchers in the development and reporting of adapted psychosocial interventions in schizophrenia. Our framework covers the nature of cultural adaptations. A fruitful avenue for future research is to review and develop guidelines relating to the process of cultural adaptation. This has recently been attempted in relation to depression (Chowdhary et al. 2014) and suggests that the process of adapting interventions is systematic and reliable but that information is missing in published work. Methodology relating to the nature and process of adaptation requires better reporting in future studies.

\section{Conclusion}

The degree of similarity in the process of adaptation across different psychosocial interventions allowed generation of a common framework describing cultural-adaptation 
which may be useful in developing and reporting future interventions. This was validated by the evidence that not only were adapted interventions more efficacious than usual care but also that degree of cultural adaptation was proportional to efficacy. There was substantial heterogeneity in outcome, with features of context, intervention and study design affecting efficacy that were often related and difficult to disentangle. There was insufficient evidence to conclude that culturally adapted interventions are more efficacious than unadapted ones. While improved efficacy is an important aim for interventions adapted for ethnic minorities, adaptation is a necessity for implementation in low to middle income countries. Future research warrants better designed controlled trials that compare adapted versus unadapted interventions, particularly in minority populations.

\section{Acknowledgements}

This project was supported by the National Institute for Health Research (NIHR) Health Service and Delivery Research Programme (HS\&DR) (D.E., project number: 12/5001/62).

The views and opinions expressed therein are those of the authors and do not necessarily reflect those of the Health Service and Delivery Research Programme (HS\&DR), NIHR, NHS or the Department of Health.

\section{Conflict of interest}

None 
References

Barrera, M. \& Castro, F. G. (2006). A heuristic framework for the cultural adaptation of interventions. Clinical Psychology: Science and Practice 13, 311-316.

Benish, S. G., Quintana, S. \& Wampold, B. E. (2011). Culturally adapted psychotherapy and the legitimacy of myth: a direct-comparison meta-analysis. Journal of Counseling Psychology 58, 279.

Bernal, G. \& Sáez-Santiago, E. (2006). Culturally centered psychosocial interventions. Journal of Community Psychology 34, 121-132.

Bhugra, D., Leff, J., Mallett, R., Der, G., Corridan, B. \& Rudge, S. (1997). Incidence and outcome of schizophrenia in Whites, African-Caribbeans and Asians in London. Psychological Medicine 27, 791798.

Bhui, K., Aslam, W., Palinski, A., McCabe, R., Johnson, M., Weich, S., Singh, S., Knapp, M., Ardino, V. \& Szczepura, A. (2015). Interventions to improve therapeutic communications between Black and minority ethnic patients and professionals in psychiatric services: systematic review. The British Journal of Psychiatry 207, 95-103.

Bhui, K. \& Bhugra, D. (2004). Communication with patients from other cultures: the place of explanatory models. Advances in Psychiatric Treatment 10, 474-478.

Bhui, K., Stansfeld, S., Hull, S., Priebe, S., Mole, F. \& Feder, G. (2003). Ethnic variations in pathways to and use of specialist mental health services in the UK. Systematic review. British Journal of Psychiatry 182, 105-116.

Bradley, G. M., Couchman D Psych, G. M., Perlesz, A., Nguyen, A. T., Singh, B. \& Riess, C. (2006). Multiple-family group treatment for English-and Vietnamese-speaking families living with schizophrenia. Psychiatric services 57, 521-530.

Braun, V. \& Clarke, V. (2006). Using thematic analysis in psychology. Qualitative research in psychology 3, 77-101.

Brockwell, S. E. \& Gordon, I. R. (2001). A comparison of statistical methods for meta-analysis. Statistics in medicine 20, 825-840.

Brown, G., Marshall, M., Bower, P., Woodham, A. \& Waheed, W. (2014). Barriers to recruiting ethnic minorities to mental health research: a systematic review. International Journal of Methods in Psychiatric Research 23, 36-48.

Cantor-Graae, E. (2007). Ethnic minority groups, particularly African-Caribbean and Black African groups, are at increased risk of psychosis in the UK. Evidence Based Mental Health 10, 95-95.

Cantor-Graae, E. \& Selten, J. P. (2005). Schizophrenia and migration: a meta-analysis and review. American Journal of Psychiatry 162, 12-24.

Carrà, G., Montomoli, C., Clerici, M. \& Cazzullo, C. L. (2007). Family interventions for schizophrenia in Italy: randomized controlled trial. European archives of psychiatry and clinical neuroscience 257, 23-30.

Carter, L., Read, J., Pyle, M. \& Morrison, A. P. (2016). The Impact of Causal Explanations on Outcome in People Experiencing Psychosis: A Systematic Review. Clinical Psychology \& Psychotherapy 2, 332-347.

Chan, S. W.-C., Yip, B., Tso, S., Cheng, B.-s. \& Tam, W. (2009). Evaluation of a psychoeducation program for Chinese clients with schizophrenia and their family caregivers. Patient education and counseling 75, 67-76.

Chien, W. T. (2008). Effectiveness of psychoeducation and mutual support group program for family caregivers of chinese people with schizophrenia. The open nursing journal 2, 28-29.

Chien, W. T. \& Chan, S. W. C. (2004). One-year follow-up of a multiple-family-group intervention for Chinese families of patients with schizophrenia. Psychiatric services 55, 1276-1284.

Chien, W. T. \& Chan, S. W. C. (2013). The effectiveness of mutual support group intervention for Chinese families of people with schizophrenia: a randomised controlled trial with 24-month followup. International journal of nursing studies 50, 1326-1340. 
Chien, W. T., Chan, S. W. C. \& Thompson, D. R. (2006). Effects of a mutual support group for families of Chinese people with schizophrenia: 18-month follow-up. The British journal of psychiatry 189, 4149.

Chien, W. T. \& Lee, I. Y. M. (2010). The schizophrenia care management program for family caregivers of Chinese patients with schizophrenia. Psychiatric Services 61, 317-320.

Chien, W. T., Norman, I. \& Thompson, D. R. (2004). A randomized controlled trial of a mutual support group for family caregivers of patients with schizophrenia. International journal of nursing studies 41, 637-649.

Chien, W. T. \& Thompson, D. R. (2013). An RCT with three-year follow-up of peer support groups for Chinese families of persons with schizophrenia. Psychiatric Services 64, 997-1005.

Chien, W. T., Thompson, D. R. \& Norman, I. (2008). Evaluation of a Peer-Led Mutual Support Group for Chinese Families of People with Schizophrenia. American journal of community psychology 42, 122-134.

Chowdhary, N., Jotheeswaran, A., Nadkarni, A., Hollon, S., King, M., Jordans, M., Rahman, A., Verdeli, H., Araya, R. \& Patel, V. (2014). The methods and outcomes of cultural adaptations of psychological treatments for depressive disorders: a systematic review. Psychological Medicine 44, 1131-1146.

Concato, J., Shah, N. \& Horwitz, R. I. (2000). Randomized, controlled trials, observational studies, and the hierarchy of research designs. New England Journal of Medicine 342, 1887-1892.

Department of Health (2005). Delivering Race Equality in Mental Health Care: an action plan for reform inside and outside services and the Government's response to the Independent inquiry into the death of David Bennett. Department of Health: London.

Dixon, L., Dickerson, F., Bellack, A. S., Bennett, M., Dickinson, D., Goldberg, R. W., Lehman, A., Tenhula, W. N., Calmes, C. \& Pasillas, R. M. (2010). The 2009 schizophrenia PORT psychosocial treatment recommendations and summary statements. Schizophrenia bulletin 36, 48-70.

Edge, D., Degnan, A., Cotterill, S., Berry, K., Drake, R., Baker, J., Barrowclough, C., Hughes-Morley, A., Grey, P. \& Bhugra, D. (2016). Culturally-adapted Family Intervention (CaFI) for AfricanCaribbeans diagnosed with schizophrenia and their families: a feasibility study protocol of implementation and acceptability. Pilot and Feasibility Studies 2, 2-39.

Fearon, P., Kirkbride, J., Morgan, C., Dazzan, P., Morgan, K., Lloyd, T., Hutchinson, G., Tarrant, J., Fung, W., Holloway, J., Mallett, R., Harrison, G., Leff, J., Jones, P. \& Murray, R. (2006). Incidence of schizophrenia and other psychoses in ethnic minority groups: results from the MRC AESOP Study. Psychological Medicine 36, 1541-1550.

Feigin, V. (2016). Global, Regional, and National Life Expectancy, All-cause Mortality, and Causespecific Mortality for 249 Causes of Death, 1980-2015: A Systematic Analysis for the Global Burden of Disease Study 2015. The Lancet 388, 1459-1544.

Gohar, S. M., Hamdi, E., Lamis, A., Horan, W. P. \& Green, M. F. (2013). Adapting and evaluating a social cognitive remediation program for schizophrenia in Arabic. Schizophrenia research 148, 12-17.

Griner, D. \& Smith, T. B. (2006). Culturally adapted mental health intervention: A meta-analytic review. Psychotherapy: Theory, research, practice, training 43, 531-548.

Guo, X., Zhai, J., Liu, Z., Fang, M., Wang, B., Wang, C., Hu, B., Sun, X., Lv, L. \& Lu, Z. (2010). Effect of antipsychotic medication alone vs combined with psychosocial intervention on outcomes of earlystage schizophrenia: A randomized, 1-year study. Archives of General Psychiatry 67, 895-904.

Habib, N., Dawood, S., Kingdon, D. \& Naeem, F. (2015). Preliminary evaluation of culturally adapted CBT for psychosis (CA-CBTp): findings from developing culturally-sensitive CBT project (DCCP). Behavioural and cognitive psychotherapy 43, 200-208.

Hedges, L. V. \& Olkin, I. (2014). Statistical methods for meta-analysis. Academic press: Orlando, Florida. 
Higgins, J., Altman, D., Gøtzsche, P., Jüni, P., Moher, D., Oxman, A., Savović, J., Schulz, K., Weeks, L. \& Sterne, J. (2011). The Cochrane Collaboration's tool for assessing risk of bias in randomised trials. British Medical Journal 343, d5928.

Higgins, J. \& Green, S. (2008). Cochrane handbook for systematic reviews of interventions. John Wiley and Sons: New Jersey, USA.

Higgins, J. \& Thompson, S. (2002). Quantifying heterogeneity in a meta-analysis. Statistics in medicine 21, 1539-1558.

Huey, S. J., Jr. \& Polo, A. J. (2008). Evidence-based psychosocial treatments for ethnic minority youth. Journal of Clinical Child Adolescent Psychology 37, 262-301.

Hussain-Gambles, M., Atkin, K. \& Leese, B. (2004). Why ethnic minority groups are under-represented in clinical trials: A review of the literature. Health \& social care in the community 12, 382-388.

Hwang, W. C. (2009). The Formative Method for Adapting Psychotherapy (FMAP): A communitybased developmental approach to culturally adapting therapy. Professional Psychology Research and Practice 40, 369-377.

Keating, F., Robertson, D., McCulloch, A. \& Francis, E. (2002). Breaking the circles of fear: A review of the relationship between mental health services and African and Caribbean communities. The Sainsbury Centre for Mental Health: London.

Kontopantelis, E. \& Reeves, D. (2010). Performance of statistical methods for meta-analysis when true study effects are non-normally distributed: a simulation study. Statistical methods in medical research 21, 409-426.

Koolaee, A. K. \& Etemadi, A. (2009). The outcome of two family interventions for the mothers of schizophrenia patients in Iran. International Journal of Social Psychiatry 56, 634-646.

Kopelowicz, A., Zarate, R., Smith, V. G., Mintz, J. \& Liberman, R. P. (2003). Disease management in Latinos with schizophrenia: a family-assisted, skills training approach. Schizophrenia Bulletin 29, 211227.

Kopelowicz, A., Zarate, R., Wallace, C. J., Liberman, R. P., Lopez, S. R. \& Mintz, J. (2012). The ability of multifamily groups to improve treatment adherence in Mexican Americans with schizophrenia. Archives of General Psychiatry 69, 265-273.

Kulhara, P., Chakrabarti, S., Avasthi, A., Sharma, A. \& Sharma, S. (2009). Psychoeducational intervention for caregivers of Indian patients with schizophrenia: a randomised-controlled trial. Acta Psychiatrica Scandinavica 119, 472-483.

Kumar, D., Zia UI Haq, M., Dubey, I., Dotivala, K. N., Veqar Siddiqui, S., Prakash, R., Abhishek, P. \& Nizamie, S. H. (2010). Effect of meta-cognitive training in the reduction of positive symptoms in schizophrenia. European Journal of Psychotherapy and Counselling 12, 149-158.

Kung, W. W., Tseng, Y.-F., Wang, Y., Hsu, P.-C. \& Chen, D. (2012). Pilot study of ethnically sensitive family psychoeducation for Chinese-American patients with schizophrenia. Social Work in Mental Health 10, 384-408.

Lak, D. C., Tsang, H. W., Kopelowicz, A. \& Liberman, R. P. (2010). Outcomes of the Chinese Basic Conversation Skill Module (CBCSM) for people with schizophrenia having mild to moderate symptoms and dysfunction in Hong Kong. International journal of psychiatry in clinical practice 14, 137-144.

Li, Z. \& Arthur, D. (2005). Family education for people with schizophrenia in Beijing, China. The British Journal of Psychiatry 187, 339-345.

Lin, E. C.-L., Chan, C. H., Shao, W.-C., Lin, M.-F., Shiau, S., Mueser, K. T., Huang, S.-C. \& Wang, H.-S. (2013). A randomized controlled trial of an adapted Illness Management and Recovery program for people with schizophrenia awaiting discharge from a psychiatric hospital. Psychiatric rehabilitation journal 36, 243-249. 
Mann, S. K. F. \& Chong, B. B. W. (2004). The effect of symptoms coping program in reducing psychotic symptoms and enhancing functions in chinese with schizophrenia. American Journal of Psychiatric Rehabilitation 7, 69-82.

Mausbach, B. T., Bucardo, J., McKIBBIN, C. L., Goldman, S. R., Jeste, D. V., Patterson, T. L., Cardenas, V. \& Barrio, C. (2008). Evaluation of a culturally tailored skills intervention for Latinos with persistent psychotic disorders. American journal of psychiatric rehabilitation 11, 61-75.

Moher, D., Liberati, A., Tetzlaff, J., Altman, D. G. \& Group, P. (2009). Preferred reporting items for systematic reviews and meta-analyses: the PRISMA statement. British Medical Journal 339, b2535.

Naeem, F., Saeed, S., Irfan, M., Kiran, T., Mehmood, N., Gul, M., Munshi, T., Ahmad, S., Kazmi, A. \& Husain, N. (2015). Brief culturally adapted CBT for psychosis (CaCBTp): a randomized controlled trial from a low income country. Schizophrenia research 164, 143-148.

National Institute for Health and Care Excellence (2014). Psychosis and schizophrenia in adults: treatment and management. NICE clinical guidelines. Department of Health: London.

Patterson, T. L., Bucardo, J., McKibbin, C. L., Mausbach, B. T., Moore, D., Barrio, C., Goldman, S. R. \& Jeste, D. V. (2005). Development and pilot testing of a new psychosocial intervention for older Latinos with chronic psychosis. Schizophrenia Bulletin 31, 922-930.

Pfammatter, M., Junghan, U. M. \& Brenner, H. D. (2006). Efficacy of Psychological Therapy in Schizophrenia: Conclusions From Meta-analyses. Schizophrenia Bulletin 32, S64-S80.

Pharoah, F., Mari, J., Rathbone, J. \& Wong, W. (2010). Family intervention for schizophrenia. Cochrane Database of Systematic Reviews. Issue 12. The Cochrane Collaboration: Nottingham, England.

Ran, M.-S., Xiang, M.-Z., Chan, C. L.-W., Leff, J., Simpson, P., Huang, M.-S., Shan, Y.-H. \& Li, S.-G. (2003). Effectiveness of psychoeducational intervention for rural Chinese families experiencing schizophrenia. Social psychiatry and psychiatric epidemiology 38, 69-75.

Rathod, S., Kingdon, D., Phiri, P. \& Gobbi, M. (2010). Developing culturally sensitive cognitive behaviour therapy for psychosis for ethnic minority patients by exploration and incorporation of service users' and health professionals' views and opinions. Behavioural and cognitive psychotherapy 38, 511-533.

Rathod, S., Phiri, P., Harris, S., Underwood, C., Thagadur, M., Padmanabi, U. \& Kingdon, D. (2013). Cognitive behaviour therapy for psychosis can be adapted for minority ethnic groups: A randomised controlled trial. Schizophrenia Research 143, 319-326.

Razali, M., Hasanah, C., Khan, A., Subramaniam, M. \& Salleh (2000). Psychosocial interventions for schizophrenia. Journal of Mental Health 9, 283-289.

Shin, S.-K. \& Lukens, E. P. (2002). Effects of psychoeducation for Korean Americans with chronic mental illness. Psychiatric services 53, 1125-1131.

Smith, T. B., Rodríguez, M. D. \& Bernal, G. (2011). Culture. Journal of Clinical Psychology 67, 166175.

So, S. H.-W., Chan, A. P., Chong, C. S.-Y., Wong, M. H.-M., Lo, W. T.-L., Chung, D. W.-S. \& Chan, S. S. (2015). Metacognitive training for delusions (MCTd): effectiveness on data-gathering and belief flexibility in a Chinese sample. Frontiers in psychology 6, 2-15.

Tarrier, N. \& Wykes, T. (2004). Is there evidence that cognitive behaviour therapy is an effective treatment for schizophrenia? A cautious or cautionary tale? Behav Res Ther 42, 1377-1401.

The Sainsbury Centre for Mental Health (2006). Policy Paper 6: The cost of race inequality. The Sainsbury Centre for Mental Health: London.

Torres, M. (2002). United Nations Educational Scientific and Cultural Organization (UNESCO): Universal Declaration on Cultural Diversity. In General Conference of UNESCO (ed. UNESCO): Paris, France

Tseng, W.-S. (2001). Handbook of cultural psychiatry. International Review of Psychiatry 14, 71-73. 
Valencia, M., Rascon, M. L., Juarez, F., Escamilla, R., Saracco, R. \& Liberman, R. P. (2010). Application in Mexico of psychosocial rehabilitation with schizophrenia patients. Psychiatry 73, 248263.

Valencia, M., Rascón, M. L., Juárez, F. \& Murow, E. (2007). A psychosocial skills training approach in Mexican out-patients with schizophrenia. Psychological Medicine 37, 1393-1402.

Wahass, S. \& Kent, G. (1997). The modification of psychological interventions for persistent auditory hallucinations to an Islamic culture. Behavioural and Cognitive Psychotherapy 25, 351-364.

Waheed, W., Woodham, A., Hughes-Morley, A., Allen, G. \& Bower, P. (2015). Overcoming barriers to recruiting ethnic minorities to mental health research: a typology of recruitment strategies. BioMedCentral Psychiatry 15, 101.

Wang, Y., Roberts, D. L., Xu, B., Cao, R., Yan, M. \& Jiang, Q. (2013). Social cognition and interaction training for patients with stable schizophrenia in Chinese community settings. Psychiatry research 210, 751-755.

Wykes, T., Steel, C., Everitt, B. \& Tarrier, N. (2008). Cognitive behavior therapy for schizophrenia: Effect sizes, clinical models, and methodological rigor. Schizophrenia Bulletin 34, 523-537.

Xiang, M., Ran, M. \& Li, S. (1994). A controlled evaluation of psychoeducational family intervention in a rural Chinese community. The British Journal of Psychiatry 165, 544-548.

Xiong, W., Phillips, M. R., Hu, X., Wang, R., Dai, Q., Kleinman, J. \& Kleinman, A. (1994). Familybased intervention for schizophrenic patients in China. A randomised controlled trial. The British Journal of Psychiatry 165, 239-247.

Zimmer, M., Duncan, A. V., Laitano, D., Ferreira, E. E. \& Belmonte-de-Abreu, P. (2007). A twelveweek randomized controlled study of the cognitive-behavioral Integrated Psychological Therapy program: positive effect on the social functioning of schizophrenic patients. Revista brasileira de psiquiatria 29, 140-147. 
The nature and efficacy of culturally-adapted psychosocial interventions for schizophrenia: a systematic review and meta-analysis

FIGURES IN TEXT

CONTENTS

- Figure 1: PRISMA flow diagram

- Figure 2: Forest plot for total symptoms 
TABLES AND FIGURES

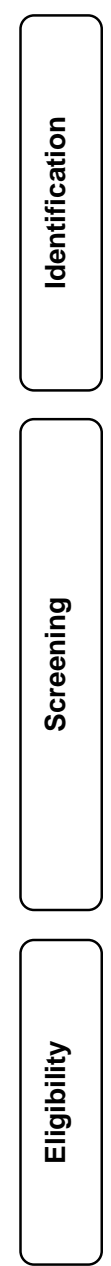

Records identified through database searching

$$
(n=4,695)
$$

Embase $(n=1,737)$, Medline $(n=763)$,

PsycINFO $(n=846)$, CINAHL $(n=1,349)$

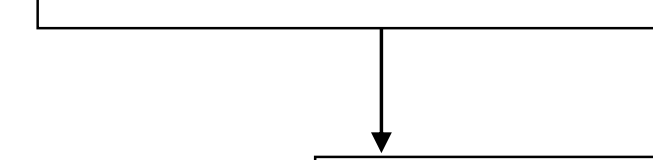

Additional records identified through searching ref lists

$$
(n=14)
$$

Records after duplicates removed

$$
(n=3,723)
$$
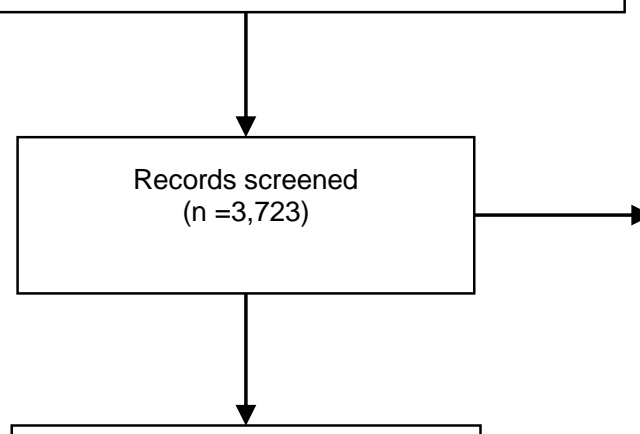

Full-text articles assessed for eligibility

$(\mathrm{n}=318)$
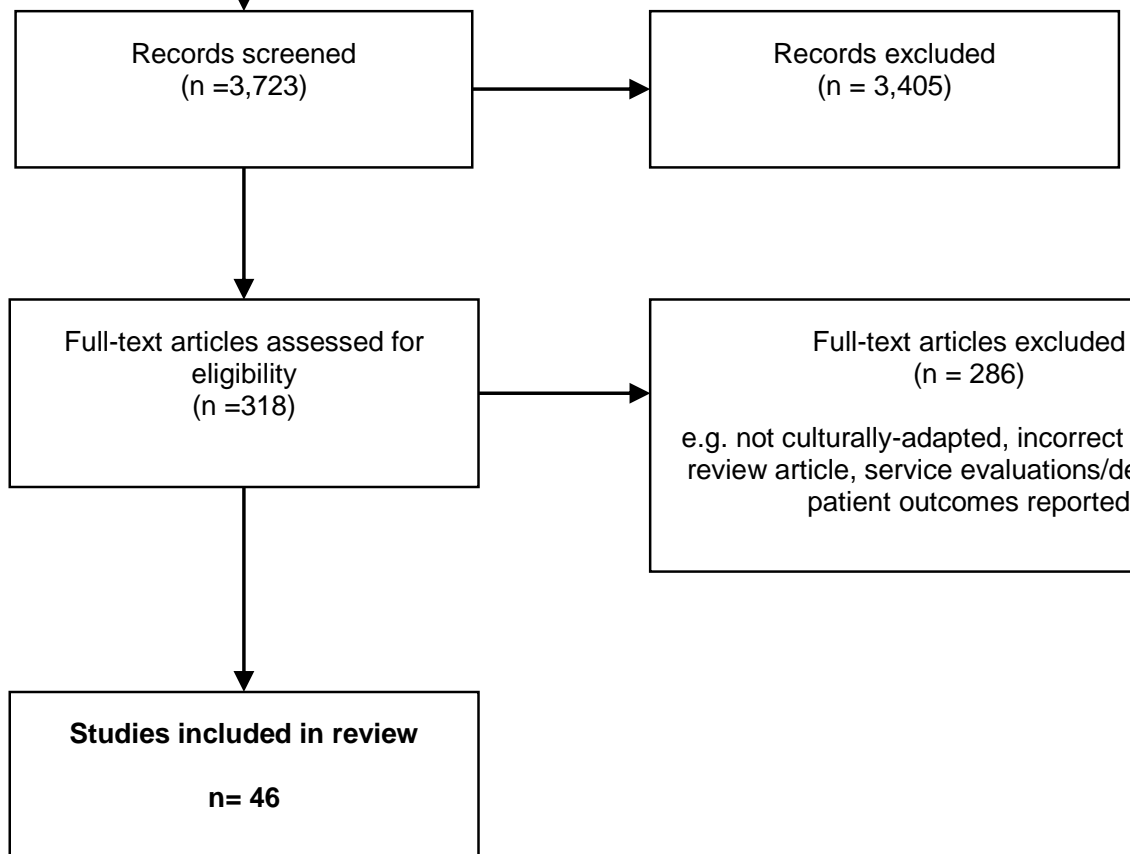

Full-text articles excluded

$$
(n=286)
$$

e.g. not culturally-adapted, incorrect diagnosis, review article, service evaluations/designs, no patient outcomes reported

Figure 1. PRISMA flow diagram 
TABLES AND FIGURES

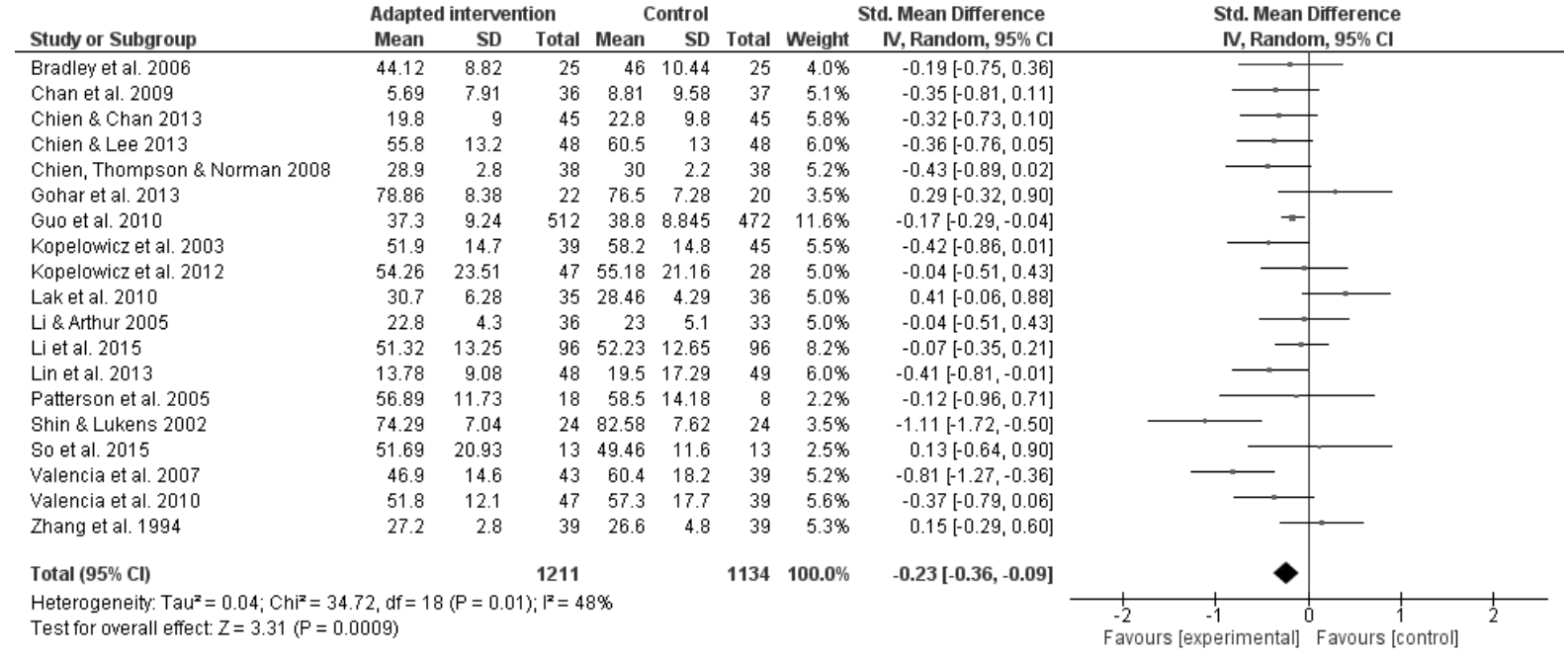

Figure 2. Forest plot of effect of culturally-adapted psychosocial interventions compared to control on total symptom severity post-treatment 
TABLES AND FIGURES

The nature and efficacy of culturally-adapted psychosocial interventions for schizophrenia: a systematic review and meta-analysis

\section{TABLES IN TEXT}

\section{CONTENTS}

- Table 1: Subgroup analyses for total symptoms 
Table 1:

Subgroup analyses of RCTs of culturally-adapted interventions for schizophrenia (total symptoms; postintervention; $n=19$ )

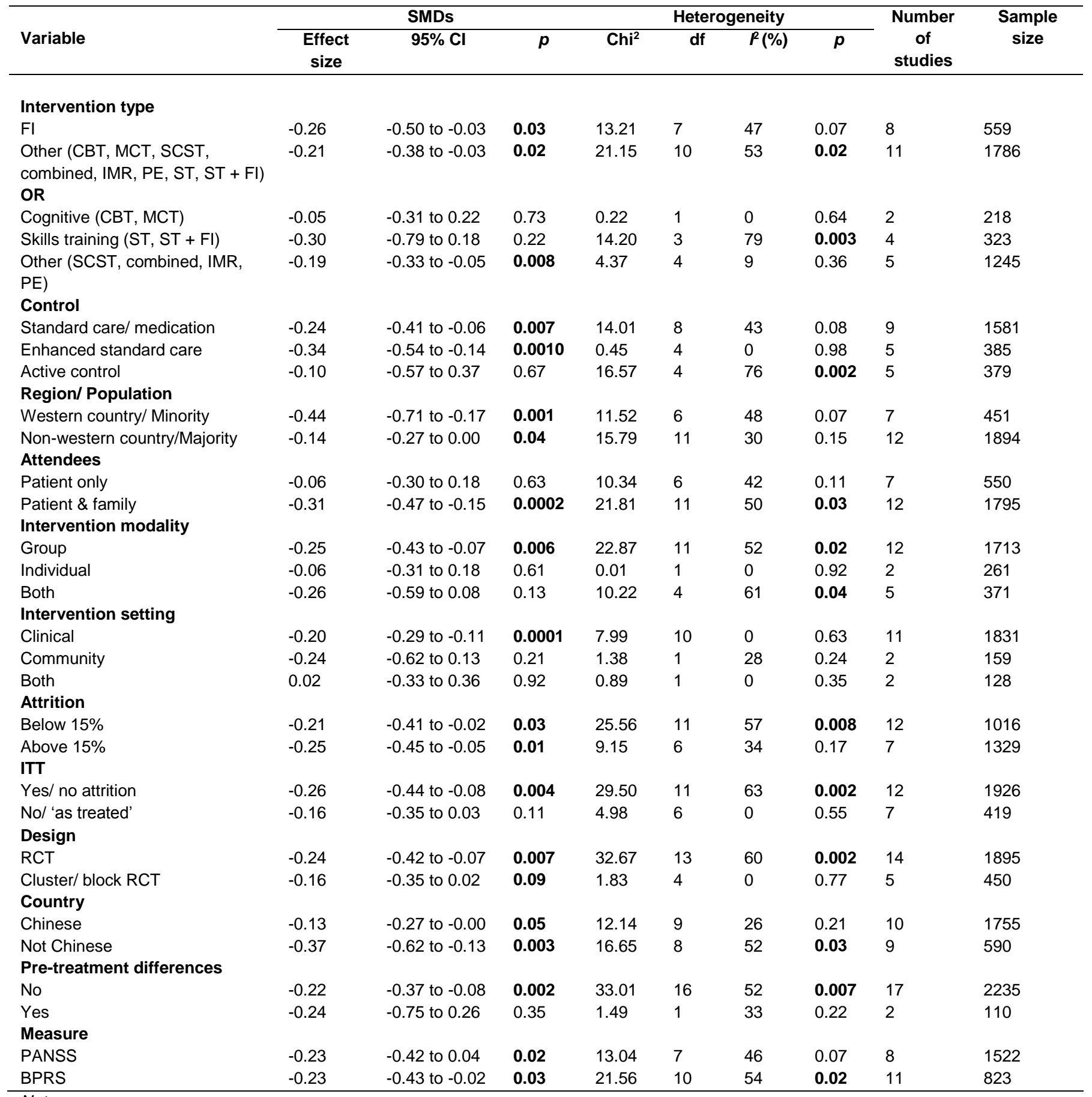

Note:

CBT=Cognitive Behavioural Therapy; MCT=Metacognitive Therapy; SCST=Social Cognitive Skills Training; IMR=IIIness Management and Recovery Programme; $P E=P$ sychoeducation; ST=Skills Training; Fl=Family Intervention; PANSS=Positive and Negative Symptom Scale; $B P R S=$ Brief Psychiatric Rating Scale; 
SUPPLEMENTARY

The nature and efficacy of culturally-adapted psychosocial interventions for schizophrenia: a systematic review and meta-analysis

\section{SUPPLEMENTARY MATERIAL}

\section{CONTENTS}

- Appendix 1: Search strategy

- Appendix 2: Risk of bias assessments

- Appendix 3: Funnel plots - publication bias

- Appendix 4: Study characteristics table

- Appendix 5: Intervention characteristics table

- Appendix 6: Culturally adapted themes and examples table

- Appendix 7: Forest plots for positive, negative and general symptoms

- Appendix 8: Subgroup analyses for positive, negative and general symptoms

- Appendix 9: Cross-tab of culturally-adapted themes against study 


\section{Appendix 1: Search strategy}

TOTAL $=$ CINAHL, PsycINFO, Medline \& EMBASE $=4695$

PsycINFO [ovid] $=846$

[PsycINFO 1806 to March Week 12016$]$

1. exp schizophrenia/ or exp psychosis/

2. (psychotic or schizo* or psychosis or psychoses).sh.ti.ab.hw.id [subject heading, title, abstract, heading word, key concepts]

3. ((chronic ${ }^{*}$ or sever $\left.{ }^{\star}\right)$ adj5 mental ${ }^{*}$ adj5 (ill ${ }^{*}$ or disorder $\left.\left.{ }^{\star}\right)\right)$. sh.ti.ab.hw.id.

4. or/1-3

5. exp ethnology/ or exp culture (Anthropological) / or exp racial and ethnic groups/ or exp racial and ethnic differences/ or exp cross-cultural differences/ exp sociocultural factors/ or exp cultural sensitivity/ or exp acculturation/ or exp minority groups

6. (ethnic ${ }^{*}$ or ethnolog ${ }^{*}$ or cultur ${ }^{*}$ or acculturation or rac $^{*}$ or minorit ${ }^{*}$ or transcultur ${ }^{*}$ or sociocultur $\left.{ }^{\star}\right)$.sh.ti.ab.hw.id.

7. ((cultur ${ }^{\star}$ adj3 (identity or cross or specific or sensiti* or relevant or adapt ${ }^{\star}$ or competen* ${ }^{\star}$ or divers*)).sh.ti.ab.hw.id.

8. ((ethnic or racial) adj3 (identity or group or minorit* or difference or variation or divers $\left.{ }^{\star}\right)$ ). sh.ti.ab.hw.id.

9. or $/ 5-8$

10. exp cross-cultural treatment/ or exp psychosocial rehabilitation/ or exp mental health services/ or exp psychotherapy/ or exp behaviour therapy/ or exp sociotherapy

11. ((psychological or psychosocial or psychiat ${ }^{\star}$ or clinical) adj5 (intervention or therap* or rehabilitation or treatment or care)).sh.ti.ab.hw.id.

12. ((cognitive or behav ${ }^{*}$ or famil ${ }^{*}$ or systemic or social ${ }^{*}$ or education*) adj5 (intervention or therap ${ }^{*}$ or rehabilitation or treatment or training or skill $)$ ).sh.ti.ab.hw.id.

13. ((cognitive or behav*) adj5 (remediat*).sh.ti.ab.hw.id.

14. ((evidence based or empirically supported) adj5 (intervention or therap* or treatment)). sh.ti.ab.hw.id.

15. or/10-14

16. exp clinical trials/ or exp treatment effectiveness evaluation/

17. exp experimental controls

18. (controlled or clinical) adj3 (trial). sh.ti.ab.hw.id. 
19. (random* or control $^{*}$ or trial or condition or assigned or group). sh.ti.ab.hw.id.

20. or/ 16-19

21.4 and 9 and 15 and 20

\section{Medline $[$ ovid $]=763$}

\section{[Ovid MEDLINE(R) 1946 to February Week 4 2016]}

1. exp schizophrenia/ or exp psychotic disorders/

2. (psychotic or schizo* or psychosis or psychoses).ab.hw.kf.ti.kw [abstract, subject heading word, keyword heading word, title, keyword heading]

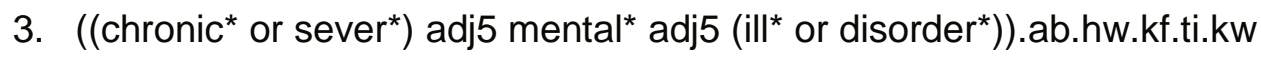

4. or/1-3

5. exp culture/ or exp ethnology/ or exp acculturation/ or exp cross-cultural comparison/ or exp cultural characteristics/ or exp cultural diversity/ or exp ethnic groups/ or minority groups

6. (ethnic* or ethnolog* or cultur* or accultur* or rac* or minorit* or transcultur* or sociocultur $\left.{ }^{\star}\right) \cdot a b . h w . k f . t i . k w$

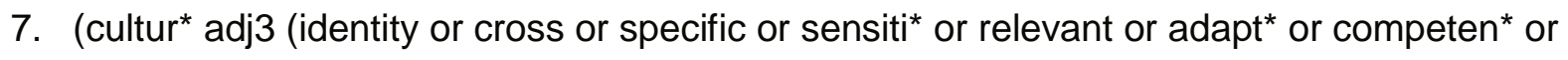
divers $\left.\left.{ }^{*}\right)\right)$. ab.hw.kf.ti.kw

8. ((ethnic or racial) adj3 (identity or group* or minorit* or difference or variation or divers $\left.{ }^{\star}\right)$ ). ab.hw.kf.ti.kw

9. $\operatorname{or} / 5-8$

10. exp rehabilitation/ or exp mental health services/ or exp psychotherapy/ or exp behaviour therapy

11. ((psychological or psychosocial or psychiat ${ }^{\star}$ or clinical) adj5 (intervention or therap* or rehabilitation or treatment or care)). ab.hw.kf.ti.kw

12. ((cognitive or behav* ${ }^{\star}$ or famil ${ }^{\star}$ or systemic or social ${ }^{\star}$ or education*) adj5 (intervention or therap* or rehabilitation or treatment or training or skill*)). ab.hw.kf.ti.kw

13. ((cognitive or behav $\left.{ }^{\star}\right)$ adj5 (remediat*). ab.hw.kf.ti.kw

14. ((evidence based or empirically supported) adj5 (intervention or therap* or treatment)). ab.hw.kf.ti.kw

15. or/10-14

16. exp clinical trial/ or exp controlled clinical trial/ or exp randomized controlled trial

17. exp random allocation/ or exp double-blind method/ or exp single blind method/ or exp control groups

18. (controlled or clinical) adj3 (trial). ab.hw.kf.ti.kw

19. (random* or control $^{*}$ or trial or condition or assigned or group). ab.hw.kf.ti.kw 
20. exp feasibility studies/ exp pilot projects/ exp intervention studies

21. or/16-20

22. 4 and 9 and 15 and 21

\section{Embase [ovid] $=1737$}

\section{[Embase 1980 to 2016 Week 10]}

1. exp schizophrenia/ or exp psychosis/

2. (psychotic or schizo $\$$ or psychoses or psychosis).ti.hw.ab.kw.sh [title, heading words, abstract, key word, subject headings]

3. ((chronic $\$$ or sever\$) adj5 mental\$ adj5 (ill\$ or disorder\$)).ti.hw.ab.kw.sh [title, heading words, abstract, key word, subject headings]

4. or/1-3

5. exp ethnology/ or exp ethnic group/ or exp cultural factor/ or exp minority group/ or exp cultural anthropology/ exp ethnic and racial groups/ exp cultural competence

6. (ethnic $\$$ or ethnolog $\$$ or cultur $\$$ or accultur $\$$ or rac $\$$ or minorit $\$$ or transcultur $\$$ or sociocultur\$).ti.hw.ab.kw.sh

7. (cultur $\$$ adj3 (identity or cross or specific or sensiti $\$$ or relevant or adapt $\$$ or competen $\$$ or divers\$)).ti.hw.ab.kw.sh

8. ((ethnic or racial) adj3 (identity or group\$ or minorit\$ or difference or variation or divers\$)).ti.hw.ab.kw.sh

9. $\operatorname{or} / 5-9$

10. exp psychiatric treatment/ or exp psychotherapy/ or exp sociotherapy/ or exp mental health services/ or exp psychosocial rehabilitation/ or exp behaviour therapy

11. ((psychological or psychosocial or psychiat\$ or clinical) adj5 (intervention or therap $\$$ or rehabilitation or treatment or care)). ti.hw.ab.kw.sh

12. ((cognitive or behav $\$$ or famil $\$$ or systemic or social $\$$ or education $\$$ ) adj5 (intervention or therap\$ or rehabilitation or treatment or training or skill\$)). ti.hw.ab.kw.sh

13. ((cognitive or behav\$) adj5 (remediat\$). ti.hw.ab.kw.sh

14. (evidence based or empirically supported) adj5 (intervention or therap\$ or treatment). ti.hw.ab.kw.sh

15. or $/ 10-14$

16. exp clinical trials (topic)/ or exp controlled clinical trial/ or exp randomized controlled trial 17. exp randomisation/ or exp control group 
18. (controlled or clinical) adj3 (trial). ti.hw.ab.kw.sh

19. (random $\$$ or control\$ or trial or condition or assigned or group).ti.ab

20. exp intervention study/ exp pilot study/ exp feasibility study

21. or/ $16-20$

22. 4 and 9 and 15 and 21

\section{CINAHL $[$ EBSCO host $]=1349$}

\section{MH = exact subject heading, $M M=$ exact major subject heading}

TX = keyword

1. (MH "Schizophrenia+") OR (MH "Psychotic Disorders+")

2. $\mathrm{TX}=($ psychotic $\mathrm{OR}$ schizo* OR psychosis OR psychoses)

3. $\mathrm{TX}=\left(\left(\right.\right.$ chronic $^{\star}$ OR sever $\left.{ }^{\star}\right)$ N5 mental ${ }^{\star}$ N5 $\left(\right.$ ill $^{\star}$ OR disorder $\left.\left.{ }^{\star}\right)\right)$

4. $\mathrm{OR} / 1-3$

5. (MH "Culture+") OR (MM "Acculturation") OR (MM "Cultural Diversity") OR (MM "Ethnic Groups") OR (MM "Ethnology") OR (MM "Minority Groups") OR (MM "Cultural Competence")

6. $\mathrm{TX}=\left(\right.$ ethnic $^{*} \mathrm{OR}$ ethnolog* OR cultur* OR accultur ${ }^{*} \mathrm{OR}$ rac $^{*} \mathrm{OR}$ minorit* OR transcultur* ORsociocultur*)

7. $\mathrm{TX}=\left(\right.$ cultur $^{*} \mathrm{~N} 3$ (identity OR cross OR specific OR sensiti ${ }^{\star}$ OR relevant OR adapt ${ }^{\star}$ OR competen* OR divers*))

8. $\mathrm{TX}=(($ ethnic OR racial) $\mathrm{N} 3$ (identity OR group* OR minorit* OR difference OR variation OR divers*))

9. $\mathrm{OR} / 5-8$

10. (MH "Rehabilitation, Psychosocial+") OR (MH "Mental Health Services+") OR (MH "Psychotherapy+") OR (MH "Behavior Therapy+")

11. $\mathrm{TX}=(($ psychological OR psychosocial OR psychiat* OR clinical) N5 (intervention OR therap* OR rehabilitation OR treatment OR care))

12. $\mathrm{TX}=(($ cognitive OR behav* OR famil* OR systemic OR social* OR education*)N5 (intervention OR therap* OR rehabilitation OR treatment OR training OR skill*))

13. $\mathrm{TX}=\left(\left(\right.\right.$ cognitive OR behav $\left.^{\star}\right)$ N5 $\left(\right.$ remediat $\left.\left.^{\star}\right)\right)$

14. $\mathrm{TX}=(($ (evidence based" OR "empirically supported”) N5 (intervention OR therap* OR treatment))

15. OR/10-14 
16. (MH "Clinical Trials+") OR (MM "Double-Blind Studies") OR (MM "Intervention Trials") OR (MM "Triple-Blind Studies") OR (MM "Therapeutic Trials") OR (MM "Single-Blind Studies") OR (MM "Randomized Controlled Trials")

17. (MM "Random Assignment") OR (MM "Control Group")

18. $\mathrm{TX}=(($ controlled OR clinical $) \mathrm{N} 3$ (trial) $)$

19. $\mathrm{TX}=\left(\right.$ random $^{*} \mathrm{OR}$ control $^{*} \mathrm{OR}$ trial OR condition OR assigned OR group)

20. $T X=$ ("intervention study" OR "pilot study" OR "feasibility study") OR MH "Feasibility Study"

21. OR/16-20

22. 4 and 9 and 15 and 21 
Appendix 2: Risk of bias assessments

\section{A2.1: Risk of bias graph}

Random sequence generation (selection bias)

Allocation concealment (selection bias)

Blinding of participants and personnel (performance bias)

Blinding of outcome assessment (detection bias)

Incomplete outcome data (attrition bias)

Selective reporting (reporting bias)

Other bias
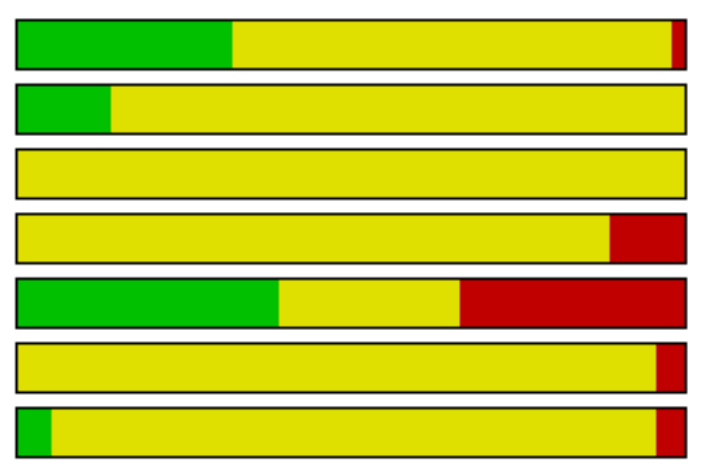

\begin{tabular}{|lllll|}
\hline $0 \%$ & $25 \%$ & $50 \%$ & $75 \%$ & $100 \%$
\end{tabular} 


\section{A2.2: Risk of bias summary}

\begin{tabular}{|c|c|c|c|c|c|c|c|}
\hline & 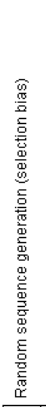 & 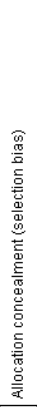 & 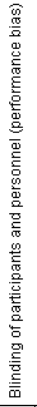 & 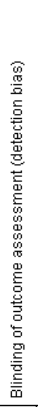 & 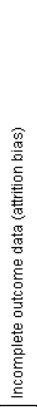 & 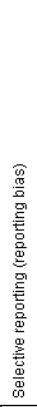 & 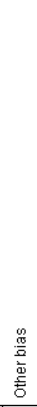 \\
\hline Bradley et al. 2006 & $?$ & $?$ & $?$ & $?$ & - & $\theta$ & $\odot$ \\
\hline Carra et al. 2007 & $\odot$ & + & $?$ & $?$ & $\odot$ & $?$ & $\odot$ \\
\hline Chan et al. 2009 & $\odot$ & $?$ & $?$ & $?$ & $\odot$ & $?$ & $?$ \\
\hline Chien, Chan \& Thompson 2006 & $?$ & $\odot$ & $?$ & $?$ & $\odot$ & $?$ & $?$ \\
\hline Chien, Norman \& Thompson 2004 & $\odot$ & $\odot$ & $?$ & $?$ & $\odot$ & $?$ & $?$ \\
\hline Chien, Thompson \& Norman 2008 & † & $\odot$ & $?$ & $?$ & $\odot$ & $?$ & $?$ \\
\hline Chien \& Chan 2004 & $?$ & $?$ & $?$ & $?$ & $\odot$ & $?$ & $?$ \\
\hline Chien \& Chan 2013 & $?$ & $\odot$ & $?$ & $?$ & $\odot$ & $?$ & $?$ \\
\hline Chien \& Lee 2010 & $?$ & $?$ & $?$ & $?$ & $\odot$ & $?$ & $?$ \\
\hline Chien \& Lee 2013 & $?$ & $?$ & $?$ & $?$ & $\odot$ & $?$ & $?$ \\
\hline Chien \& Thompson 2013 & $?$ & $?$ & $?$ & $?$ & + & $?$ & $?$ \\
\hline Chien \& Wong 2007 & $?$ & $?$ & $?$ & $?$ & $?$ & $?$ & $?$ \\
\hline Chien 2008 & $\odot$ & $\odot$ & $?$ & $?$ & $\odot$ & $?$ & $?$ \\
\hline Gohar et al. 2013 & $?$ & $?$ & $?$ & ○ & $?$ & $?$ & $?$ \\
\hline Guo et al. 2010 & $?$ & $?$ & $?$ & $?$ & $\odot$ & $?$ & 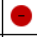 \\
\hline Habib et al. 2015 & $\odot$ & $?$ & $?$ & $?$ & $?$ & $?$ & 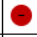 \\
\hline Koolaee \& Etemedi 2009 & $?$ & $?$ & $?$ & $?$ & - & ? & $?$ \\
\hline Kopelowicz et al. 2003 & $?$ & $?$ & $?$ & $?$ & $\Theta$ & $?$ & $?$ \\
\hline Kopelowicz et al. 2012 & 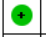 & $?$ & $?$ & $?$ & $\Theta$ & $?$ & $?$ \\
\hline Kulhara et al. 2009 & 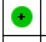 & $?$ & $?$ & ○ & ? & $?$ & $?$ \\
\hline Kumar et al. 2010 & $\odot$ & $?$ & $?$ & $?$ & $?$ & $?$ & $?$ \\
\hline Lak et al. 2010 & $?$ & $?$ & $?$ & $?$ & $\odot$ & $?$ & $?$ \\
\hline Li \& Arthur 2005 & $?$ & $?$ & $?$ & ○ & 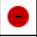 & $?$ & $?$ \\
\hline Li et al. 2015 & ๑ & $?$ & $?$ & $?$ & $\odot$ & $?$ & $?$ \\
\hline Lin et al. 2013 & $?$ & $?$ & $?$ & $\odot$ & $\odot$ & $?$ & $?$ \\
\hline Mann \& Chong 2004 & $?$ & $?$ & $?$ & $?$ & $?$ & $?$ & $?$ \\
\hline Mausbach et al. 2008 & $?$ & $?$ & $?$ & $?$ & $\odot$ & $?$ & $?$ \\
\hline Naeem et al. 2015 & $\odot$ & $?$ & $?$ & $?$ & $\odot$ & $?$ & $?$ \\
\hline Patterson et al. 2005 & \begin{tabular}{|l|}
$?$ \\
\end{tabular} & $?$ & $?$ & $?$ & - & $?$ & $?$ \\
\hline Ran et al. 2003 & $\odot$ & $?$ & $?$ & $?$ & $?$ & $?$ & $?$ \\
\hline Razali et al. 2000 & $?$ & $?$ & $?$ & $?$ & $\theta$ & $?$ & $?$ \\
\hline Shin \& Lukens 2002 & $?$ & $?$ & $?$ & $?$ & $?$ & $?$ & $?$ \\
\hline So et al. 2015 & $?$ & $?$ & $?$ & $?$ & $\theta$ & $?$ & $?$ \\
\hline Valencia et al. 2007 & \begin{tabular}{|l|l}
$?$ \\
\end{tabular} & $?$ & $?$ & $?$ & - & $?$ & $?$ \\
\hline Valencia et al. 2010 & \begin{tabular}{|l|}
$?$ \\
\end{tabular} & $?$ & $?$ & $?$ & $\odot$ & $?$ & $?$ \\
\hline Wahass \& Kent 1997 & ○ & $?$ & $?$ & 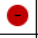 & $?$ & $?$ & $?$ \\
\hline Wang et al. 2013 & $\odot$ & $?$ & $?$ & $?$ & $\theta$ & $?$ & $?$ \\
\hline Weng et al. 2005 & $?$ & $?$ & $?$ & $?$ & $\odot$ & $?$ & $?$ \\
\hline Xiang et al. 1994 & $?$ & $?$ & $?$ & $?$ & $?$ & $?$ & $?$ \\
\hline Xiong et al. 1994 & $?$ & $?$ & $?$ & $?$ & $?$ & $?$ & $?$ \\
\hline Zhang \& Hequin 1993 & $?$ & $?$ & $?$ & $?$ & $?$ & $?$ & $?$ \\
\hline Zhang et al. 1994 & $?$ & $?$ & $?$ & $?$ & $\odot$ & $?$ & $?$ \\
\hline Zhang et al. 1998 & $?$ & $?$ & $?$ & $?$ & - & $?$ & $?$ \\
\hline Zimmer et al. 2007 & † & $?$ & $?$ & $?$ & $?$ & $?$ & $?$ \\
\hline
\end{tabular}


A3.1: Culturally-adapted intervention vs control. Total symptoms post-treatment (0-3 months)

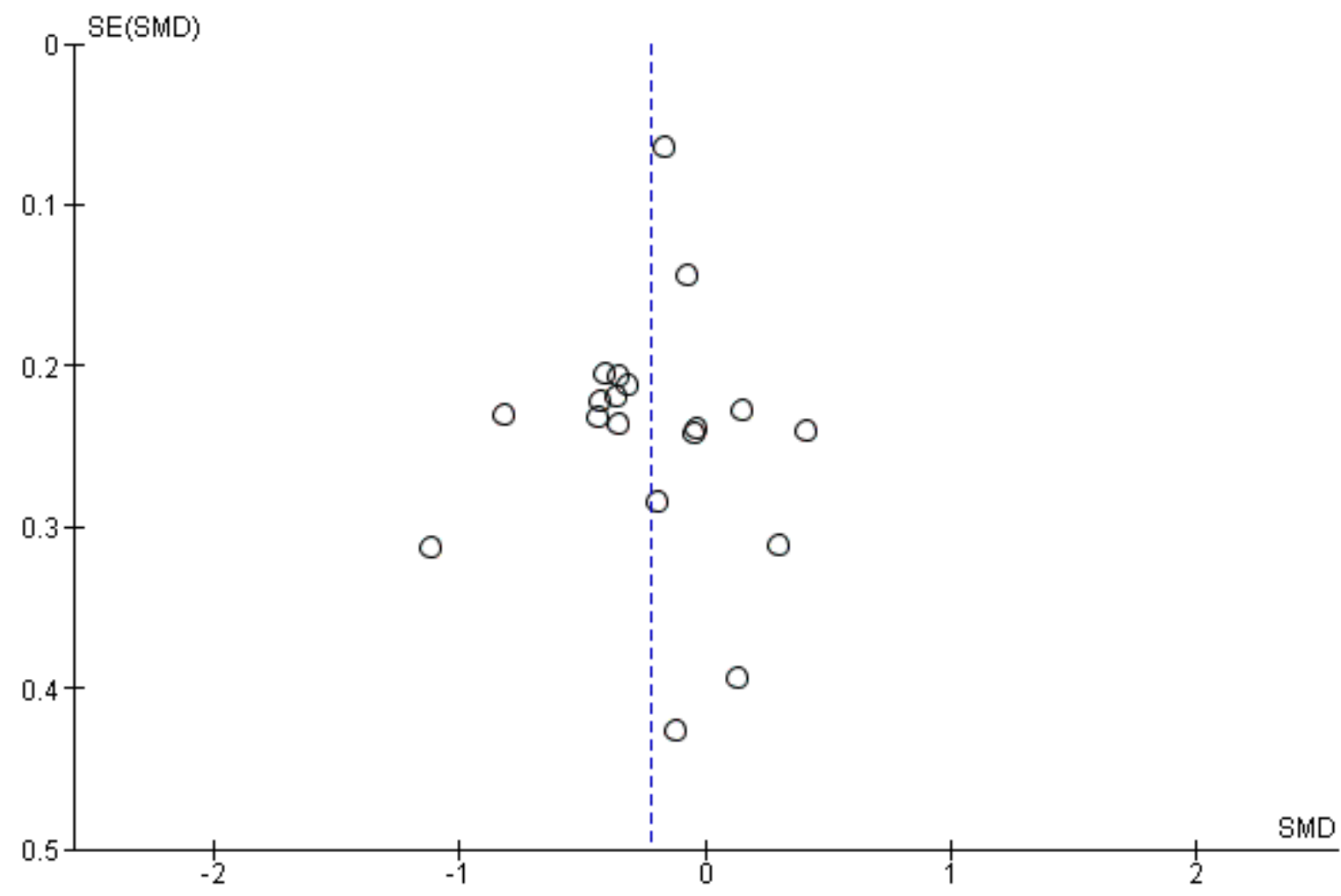

A3.2: Culturally-adapted intervention vs control. Positive symptoms post-treatment (0-3 months) 


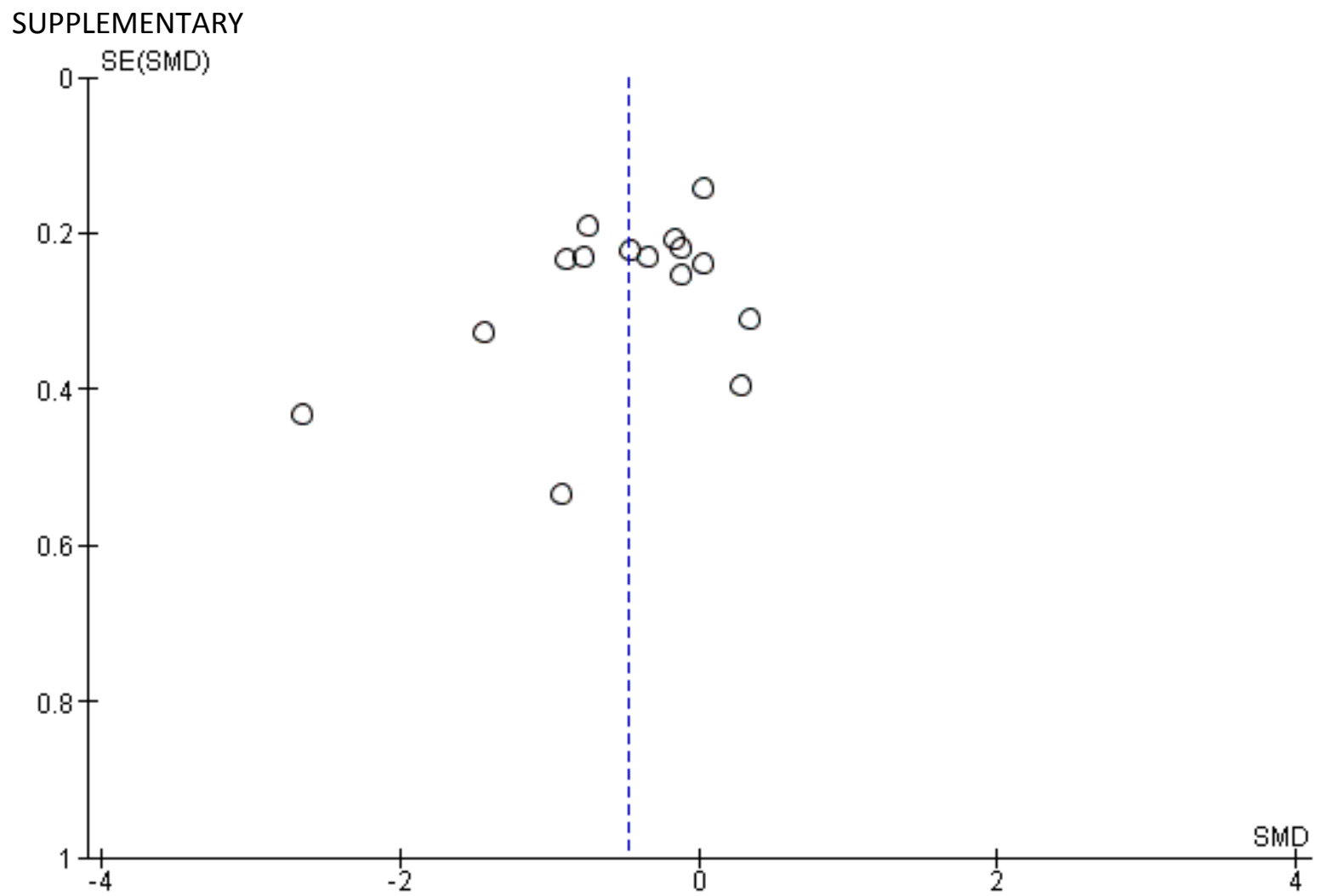

A3.3: Culturally-adapted intervention vs control. Negative symptoms post-treatment 


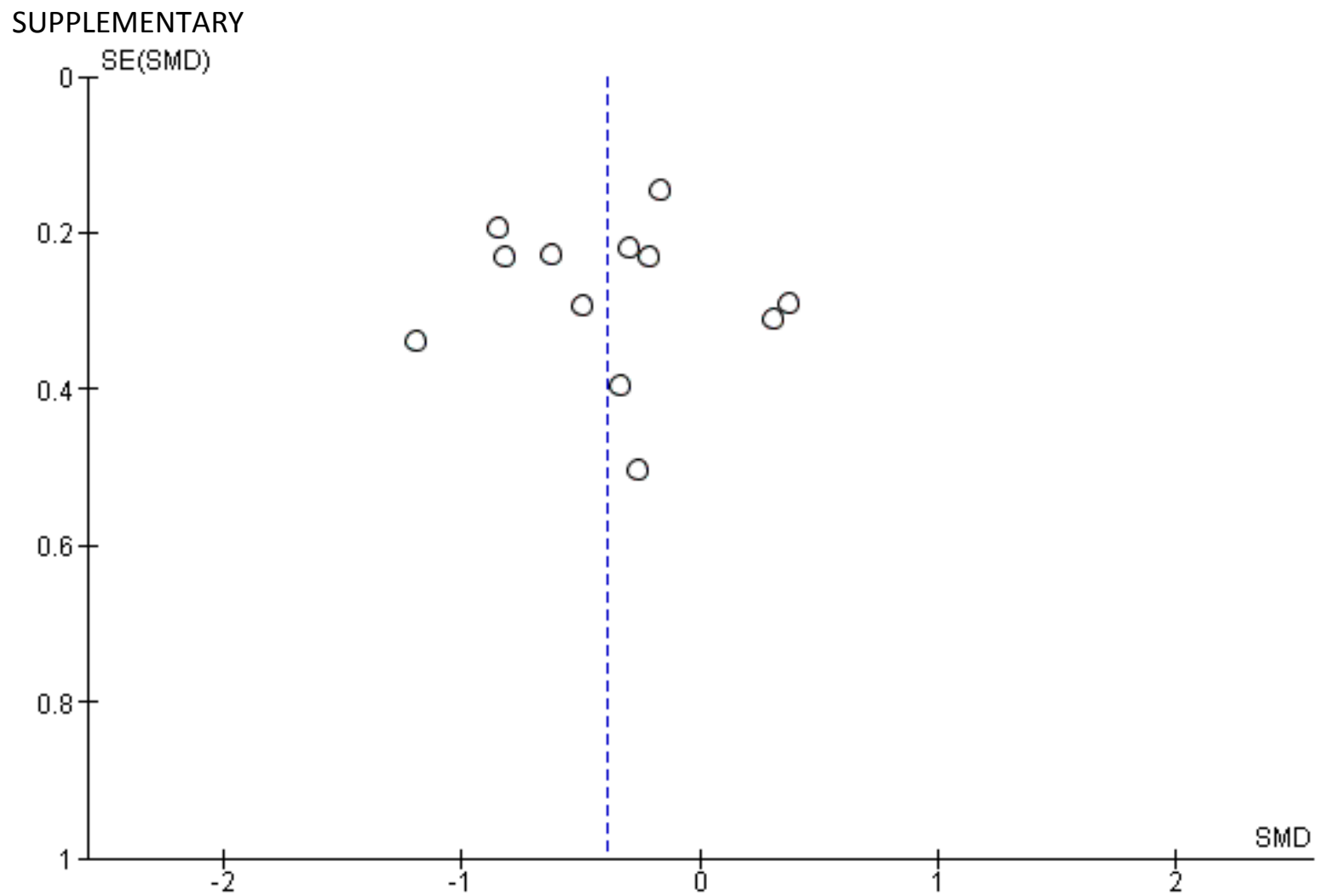

A3.4: Culturally-adapted intervention vs control. General symptoms post-treatment

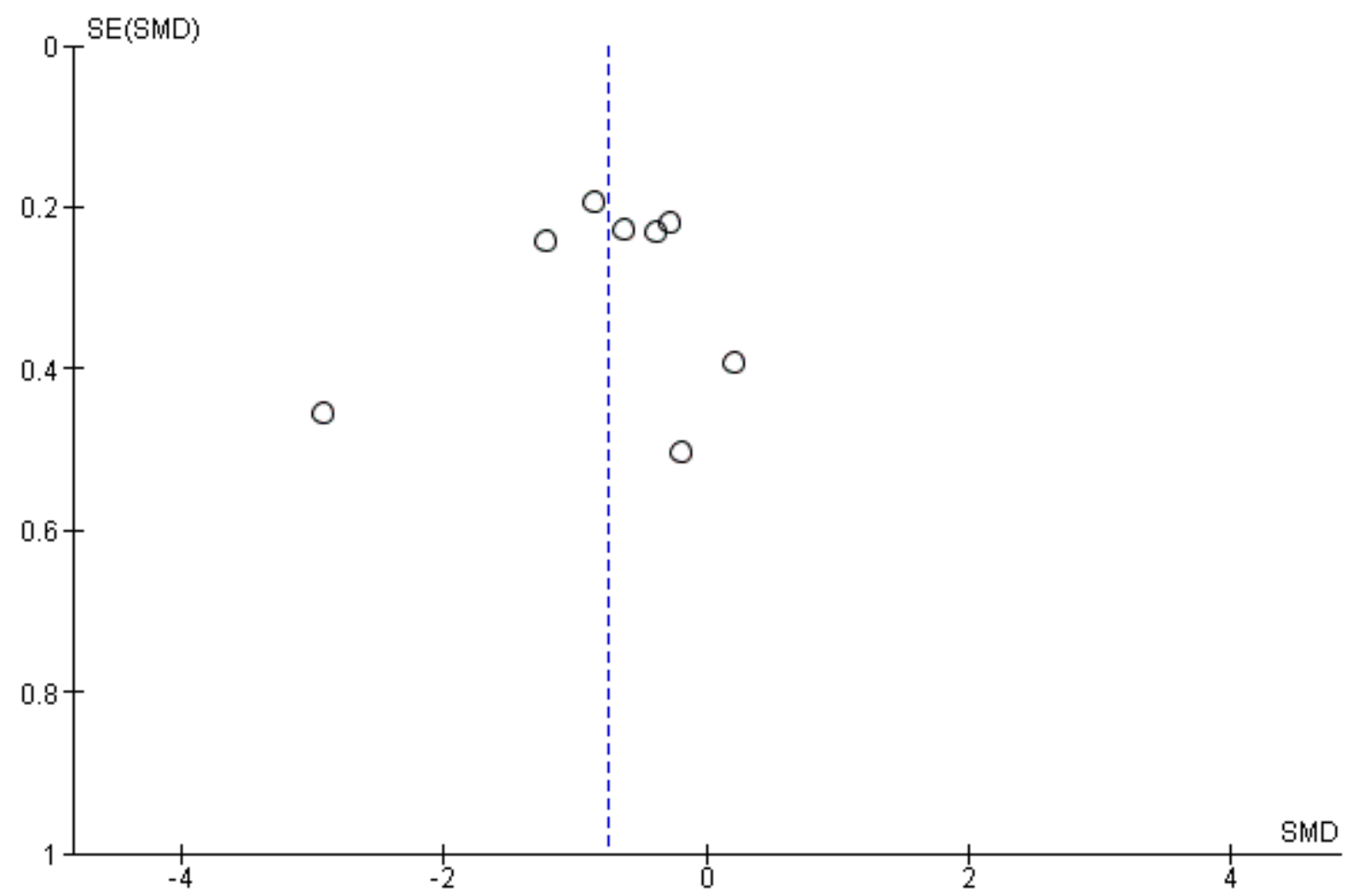




\section{Appendix 4}

Table A4: Characteristics of studies included in the systematic review of culturally-adapted interventions ( $n=46)$

\begin{tabular}{|c|c|c|c|c|c|c|c|c|c|c|c|}
\hline \multirow{2}{*}{$\begin{array}{l}\text { Author } \\
\text { (year) }\end{array}$} & \multicolumn{2}{|c|}{ Study arms $(\mathrm{N})$} & \multicolumn{4}{|c|}{ Sample characteristics } & \multirow{2}{*}{ Study setting } & \multirow{2}{*}{$\begin{array}{l}\text { Study } \\
\text { design }\end{array}$} & \multirow{2}{*}{$\begin{array}{l}\text { Patient } \\
\text { outcome } \\
\text { measures } \\
\text { (time point) }\end{array}$} & \multirow{2}{*}{$\begin{array}{l}\text { Attrition: } \\
\text { timepoint } \\
N(\%)\end{array}$} & \multirow{2}{*}{ Key findings } \\
\hline & $\begin{array}{c}\text { Adapted } \\
\text { intervention(s) }\end{array}$ & $\begin{array}{l}\text { Comparison } \\
\text { group(s) }\end{array}$ & $\begin{array}{l}\text { Gender } \\
\text { N (M/F) }\end{array}$ & Mean age & $\begin{array}{c}\text { Diagnosis } \\
\text { (diagnostic tool) }\end{array}$ & $\begin{array}{c}\text { Chronicity/ } \\
\text { duration illness } \\
\text { (yrs) }\end{array}$ & & & & & \\
\hline $\begin{array}{l}\text { Bradley et } \\
\text { al. (2006) }\end{array}$ & MFGT: 30 & $\begin{array}{l}\text { Standard } \\
\text { care - } \\
\text { enhanced: } 29\end{array}$ & $\begin{array}{l}\text { Total: } 50 \\
(15 / 35) ; \\
\text { MFGT: } 25 \\
(7 / 18) ; \\
\text { CG: } 25 \\
(8 / 17)\end{array}$ & $\begin{array}{l}\text { MFGT: } 33.6 \\
\text { CG: } 34\end{array}$ & $\begin{array}{l}\text { DSM-IV } \\
\text { outpatient } \\
\text { diagnosis } \\
\text { schizophrenia by } \\
\text { psychiatrist }\end{array}$ & $\begin{array}{l}\text { HAs } 12 \text { mths } \\
\text { pre- } \\
\text { intervention: } \\
\text { MFG: } 0.60 \text {; } \\
\text { CG: } 0.29\end{array}$ & $\begin{array}{l}\text { Community } \\
\text { mental health } \\
\text { programme of } \\
\text { the inner west } \\
\text { mental health } \\
\text { service- royal } \\
\text { Melbourne } \\
\text { hospital and } \\
\text { participant } \\
\text { home }\end{array}$ & $\mathrm{RCT}$ & $\begin{array}{l}\text { BPRS; SANS; } \\
\text { HoNOS; QOL } \\
\text { (B, P-I); } \\
\text { Relapse rates } \\
\text { (P-I, } 18 \text { mth P- } \\
\text { I) }\end{array}$ & $\begin{array}{l}18 \text { mth P-I: } \\
\text { Total: } \\
\text { 9/59 (15.3) } \\
\text { MFGT: } \\
\text { 5/30 (16.7) } \\
\text { CG: } 4 / 29 \\
(13.8)\end{array}$ & $\begin{array}{l}\text { Sig reduction found } \\
\text { between baseline and P- } \\
\text { I BPRS symptom scores } \\
\text { for MFGT but no } \\
\text { difference for CG } \\
\text { Relapse rates at end } \\
\text { and } 18 \text { months P-I were } \\
\text { sig lower for MFGT } \\
\text { compared to CG. }\end{array}$ \\
\hline $\begin{array}{l}\text { Carra et al. } \\
(2007)\end{array}$ & $\begin{array}{l}\text { IG: 50; } \\
\text { IG+SG: } 26\end{array}$ & $\begin{array}{l}\text { Standard } \\
\text { care: } 25\end{array}$ & $\begin{array}{l}\text { Total: } 101 \\
(73 / 28) ; \\
\text { IG: } 50 \\
(35 / 15) ; \\
\text { IG + SG: } 26 \\
(22 / 4) ; \\
\text { CG: } 25(16 / 9)\end{array}$ & $\begin{array}{l}\text { IG: } 29.9 \\
\text { IG + SG: } \\
29.6 ; \\
\text { CG: } 29.9\end{array}$ & $\begin{array}{l}\text { DSM-IV } \\
\text { schizophrenia } \\
\text { casenotes } \\
\text { screened by RAs }\end{array}$ & $\begin{array}{l}\text { IG: } 9.6 ; \\
\text { IG + SG: } 11.3 ; \\
\text { CG: } 10.3\end{array}$ & $\begin{array}{l}\text { Family } \\
\text { advocacy and } \\
\text { support } \\
\text { agency, } \\
\text { association } \\
\text { for research } \\
\text { on } \\
\text { schizophrenia } \\
\text { (ARS), Milan. }\end{array}$ & $\mathrm{RCT}$ & $\begin{array}{l}\text { Treatment } \\
\text { compliance; } \\
\text { relapse rates, } \\
\text { HA rates } \\
\text { GAS; } \\
\text { employment } \\
\text { rates (B, } 12 \\
\text { mth \& } 24 \text { mth } \\
\text { P-I) }\end{array}$ & $\begin{array}{l}24 \text { mth P-I: } \\
\text { Total: } \\
\text { 6/101(5.9); } \\
\text { IG: } 3 / 50 \\
\text { (6); IG + } \\
\text { SG: 2/26 } \\
\text { (8); CG: } \\
\text { 1/25 (4) }\end{array}$ & $\begin{array}{l}\text { Compliance with } \\
\text { standard community } \\
\text { care sig higher at } 12 \\
\text { month } P-I \text { in the IG+SG } \\
\text { group than CG. }\end{array}$ \\
\hline $\begin{array}{l}\text { Chan et al. } \\
\text { (2009) }\end{array}$ & PE: 36 & $\begin{array}{l}\text { Standard } \\
\text { care - } \\
\text { enhanced: } 37\end{array}$ & $\begin{array}{l}\text { Total: } 73 \\
\text { (48/25); } \\
\text { PE: } 36 \\
(26 / 10) ; \\
\text { CG: } 37 \\
(22 / 15)\end{array}$ & $\begin{array}{l}\text { PE: } 34.2 \text {; } \\
\text { CG: } 36.3\end{array}$ & $\begin{array}{l}\text { DSM-IV } \\
\text { outpatient } \\
\text { diagnosis } \\
\text { schizophrenia by } \\
\text { psychiatrist }\end{array}$ & $\begin{array}{l}\text { PE: } 10.2 \text {; } \\
\text { CG: } 10.5\end{array}$ & $\begin{array}{l}\text { Psychiatric } \\
\text { out-patient } \\
\text { department of } \\
\text { a regional } \\
\text { mental health } \\
\text { hospital in } \\
\text { Hong Kong }\end{array}$ & $\begin{array}{l}\text { Cluster } \\
\text { RCT }\end{array}$ & $\begin{array}{l}\text { BPRS; ROMI; } \\
\text { ITAQ; SES (B, } \\
\text { P-I, } 6 \text { mth \& } 12 \\
\text { mth P-I) }\end{array}$ & No attrition & $\begin{array}{l}\text { Sig improvements over } \\
\text { time found for the PE } \\
\text { group in BPRS, ROMI, } \\
\text { and ITAQ scores. Sig } \\
\text { group differences were } \\
\text { found for BPRS at } 6 \mathrm{mth} \text {, } \\
\text { ROMl at P-I and at } 6 \mathrm{mth} \\
\text { P-I, and the ITAQ at } 6 \text { \& } \\
12 \text {-mths P-I. }\end{array}$ \\
\hline $\begin{array}{l}\text { Chien } \\
\text { (2008) }\end{array}$ & PESG: 34 & $\begin{array}{l}\text { Standard } \\
\text { care - } \\
\text { enhanced: } 34\end{array}$ & $\begin{array}{l}\text { Total: } 68 \\
\text { NR }\end{array}$ & $\begin{array}{l}\text { PESG: } 37.3 \text {; } \\
\text { CG: } 28.8\end{array}$ & $\begin{array}{l}\text { DSM-IV } \\
\text { schizophrenia } \\
\text { diagnosis }\end{array}$ & 2.5 & $\begin{array}{l}\text { Two } \\
\text { psychiatric } \\
\text { outpatient } \\
\text { clinics, Hong } \\
\text { Kong }\end{array}$ & $\begin{array}{l}\text { Cluster } \\
\text { RCT }\end{array}$ & $\begin{array}{l}\text { SLOF (B, } 1 \text { \& } \\
12 \text { mths P-I) } \\
\text { No. \& duration } \\
\text { HA ( } 6 \text { mth pre- } \\
\text { intervention, } \\
\text { over } 9 \text { mth } \\
\text { intervention } \\
\text { period, 12-mth } \\
\text { P-I) }\end{array}$ & $\begin{array}{l}12 \text { mth P-I: } \\
\text { Total: 5/68 } \\
\text { (7.4); } \\
\text { PESG: } \\
\text { 3/34 (8.8); } \\
\text { CG: } 2 / 34 \\
\text { (5.9) }\end{array}$ & $\begin{array}{l}\text { PESG showed sig } \\
\text { improved level of } \\
\text { functioning (SLOF) from } \\
\text { B to } 6 \text { mth P-I when } \\
\text { compared to CG. }\end{array}$ \\
\hline
\end{tabular}




\section{SUPPLEMENTARY}

\begin{tabular}{|c|c|c|c|c|c|c|c|}
\hline $\begin{array}{l}\text { Chien \& } \\
\text { Chan } \\
(2004)^{\star}\end{array}$ & $\begin{array}{l}\text { MSG: } 32 \text {; } \\
\text { PE: } 33\end{array}$ & $\begin{array}{l}\text { Standard } \\
\text { care - } \\
\text { enhanced: } 31\end{array}$ & $\begin{array}{l}\text { Total: } 96 \\
\text { (62/34); } \\
\text { MSG: } 32 \\
\text { (20/12); } \\
\text { PE: 33 } \\
\text { (20/13); } \\
\text { CG: } 31 \\
\text { (22/9) }\end{array}$ & $\begin{array}{l}\text { MSG: } 32.3 \text {; } \\
\text { PE: } 29.1 \text {; } \\
\text { CG: } 33.8\end{array}$ & $\begin{array}{l}\text { DSM-IV } \\
\text { schizophrenia } \\
\text { diagnosis }\end{array}$ & $\begin{array}{l}\text { Just over two } \\
\text { years (range: } \\
6 \text { mths-5yrs) }\end{array}$ & $\begin{array}{l}\text { Two } \\
\text { psychiatric } \\
\text { outpatient } \\
\text { clinics, Hong } \\
\text { Kong }\end{array}$ \\
\hline $\begin{array}{l}\text { Chien \& } \\
\text { Chan } \\
\text { (2013) }\end{array}$ & $\begin{array}{l}\text { MSG: } 45 ; \\
\text { PE: } 45\end{array}$ & $\begin{array}{l}\text { Standard } \\
\text { care - } \\
\text { enhanced: } 45\end{array}$ & $\begin{array}{l}\text { Total: } 135 \\
\text { (84/51); } \\
\text { MSG: } 45 \\
\text { (28/17); } \\
\text { PE: 45 } \\
\text { (27/18); } \\
\text { CG: 45 } \\
(29 / 16)\end{array}$ & $\begin{array}{l}\text { MSG: } 24.3 \text {; } \\
\text { PE: } 25.2 ; \\
\text { CG: } 26.2\end{array}$ & $\begin{array}{l}\text { DSM-IV } \\
\text { schizophrenia } \\
\text { diagnosis }\end{array}$ & $\begin{array}{l}\text { Majority } 1-2 \text { yrs } \\
\text { for all } 3 \text { groups }\end{array}$ & $\begin{array}{l}\text { Two } \\
\text { psychiatric } \\
\text { outpatient } \\
\text { clinics, Hong } \\
\text { Kong }\end{array}$ \\
\hline
\end{tabular}

\begin{tabular}{|c|c|c|c|c|c|c|c|}
\hline $\begin{array}{l}\text { Chien, } \\
\text { Chan \& } \\
\text { Thompson } \\
(2006)^{\star}\end{array}$ & $\begin{array}{l}\text { MSG: } 32 \\
\text { PE: } 33\end{array}$ & $\begin{array}{l}\text { Standard } \\
\text { care - } \\
\text { enhanced: } 31\end{array}$ & $\begin{array}{l}\text { Total: } 96 \\
\text { (64/32); } \\
\text { MSG: } 32 \\
\text { (20/12); } \\
\text { PE: 33 } \\
\text { (22/11); } \\
\text { CG: } 31 \text { (22/9) }\end{array}$ & $\begin{array}{l}\text { MS: } 27.3 \text {; } \\
\text { PE: } 27.8 ; \\
\text { CG: } 28.8\end{array}$ & $\begin{array}{l}\text { DSM-IV } \\
\text { outpatients } \\
\text { casenote } \\
\text { diagnosis } \\
\text { schizophrenia }\end{array}$ & $\begin{array}{l}\text { About } 2 \text { yrs } \\
\text { (range: } 6 \text { mths- } \\
3 \text { yrs) }\end{array}$ & $\begin{array}{l}\text { Two } \\
\text { psychiatric } \\
\text { outpatient } \\
\text { clinics, Hong } \\
\text { Kong }\end{array}$ \\
\hline
\end{tabular}

Chien \&

SCMP: 46

Lee (2010) $\begin{array}{ll}\text { Standard } & \text { Total: } 92 \\ \text { care - } & \text { NR }\end{array}$

enhanced: 46

$\begin{array}{ll}\text { NR } & \text { DSM-IV } \\ \text { schizophrenia } \\ \text { diagnosis }\end{array}$

diagnosis

Cluster
RCT

\section{NR}

Three

psychiatric

clinics, Hong

Kong
BPRS; SLOF;
(B, 1wk \& 12 (B, 1wk \&

No. \& duratio

of HA (pre-

intervention,

over $6 \mathrm{mth}$

intervention

period, 12 mth

P-I)

\section{Cluster}

RCT

BPRS; SLOF 24 mth P-I)

No. \& duration

of HA $(9 \mathrm{mth}$

pre-

intervention,

wk, 12 \& 24

mth P-I).

$12 \mathrm{mth}$ P-I: $\quad$ MSG participants

reported sig reduction in (7.3); MSG: rehospitalisation

4/32 (12.5); compared to the PE and PG: $2 / 33 \quad C G$ groups and greater (6.1); CG: improvement in all three 1/31 (3.2) subscales of SLOF (selfmaintenance, social

functioning, and

community living skills)

$24 \mathrm{mth}$ P-I: $\quad$ At $12 \mathrm{mth}$ and $24 \mathrm{mth} \mathrm{P}-$ time points compared with the CG group,

MSG: 2/45 BPRS score decreased

4.4); PG: sig and the SLOF score $3 / 45(6.7) ; \quad$ for the MSG increased CG: $2 / 45$ (4.4)

sig. Scores also

decreased at $12 \mathrm{mth}$ and 24 mth P-I when compared with PE group. The average length of re-

hospitalisations was sig lower in the MSG than $C G$ at all $P-I$ time points.

Cluster

BPRS; SLOF;

18 mth P-I: Readmissions to hospita (B, 6 mth \& 18 mth $\mathrm{P}-\mathrm{I}$ )

No. and duration of $\mathrm{HA}$ (6 mth pre7.3); MSG: in the MSG reduced sig more than the other two (6.3); groups from baseline to (6.1): CG $\begin{array}{ll}6 \text { mth pre- } & (6.1) ; C G \\ \text { intervention, } 6 & 3 / 31(9.7)\end{array}$ $\mathrm{mth} \& 18 \mathrm{mth}$ P-I)

18 mths P-I. Level of functioning in MSG improved sig from baseline to $18 \mathrm{mth} \mathrm{P}-\mathrm{I}$ compared to the other two groups. Patient functioning in the $\mathrm{PE}$ group also improved over time and sig more than the $C G$ group.

RCT BPRS; SLOF; (B, 1 mth \& 15 mth $P-I)$ No. \& duration preceding $\begin{array}{ll}\text { preceding } & \text { CG: } \\ \text { intervention, } 1 & \text { (2.2) }\end{array}$ SCMP:
There were sig differences between the SCMP and CG with patients' functioning and number and length of rehospitalisations at $1 \mathrm{mth}$
and $15 \mathrm{mth} \mathrm{P}-\mathrm{I}$, although 
mth \& 15 mth P-I)

$\begin{array}{llll}\begin{array}{l}\text { Chien \& } \\ \text { Lee (2013) }\end{array} & \text { MBPP: } 48 & \begin{array}{l}\text { Standard } \\ \text { care }-\end{array} \\ \text { enhanced: } 48\end{array} \quad \begin{aligned} & \text { Total: } 96 \\ & (53 / 43)\end{aligned}$

25.8

DSM-IV
schizophrenia
diagnosis

diagnosis enhanced: 48

\section{NR}

psychiatric
outpatient
clinics, Hong
Kong

Chien,

Norman \&

Thompson

(2004)

MSG: 24

(27/21);

MSG: 24

CG: 24

(13/11)

Chien \&
Thompson

(2013)
FPGP: 35

$\begin{array}{ll}\text { CG1: } & \text { Total: } 106 \\ \text { Standard } & (66 / 40) ; \\ \text { care - } & \text { FPGP: } 35 \\ \text { enhanced: } & (21 / 14) ; \\ \text { 35; } & \text { CG1:36 } \\ \text { CG2: psycho- } & (23 / 13) ; \\ \text { education: 36 } & \text { CG2: 35 } \\ & (22 / 13)\end{array}$

\section{MSG: 39.9; \\ CG: 36.3 \\ DSM-IV \\ schizophrenia
diagnosis}

$\begin{array}{ll}\text { FPGP: } 26.3 ; & \text { DSM-IV } \\ \text { CG1: } 28.2 ; & \text { schizophrenia } \\ \text { CG2: } 27.2 & \text { diagnosis }\end{array}$

$\begin{array}{ll}\text { Approx } 2 \text { yrs } & \text { Two } \\ & \text { psychiatric } \\ & \text { outpatient } \\ \text { clinics }\end{array}$

2.5 yrs (range: 6 mths -6 yrs)
Three

outpatient

clinics, Hong

Kong
RCT

\section{SSQS; ITAQ} (baseline, 3 mth \& $18 \mathrm{mth}$ P-I)

No. \& duration HA (6 mths preceding

intervention, 3 $\mathrm{mth} \& 18 \mathrm{mth}$ P-I)
BPRS; SLOF;

\section{Cluster Duration of \\ No attrition} HA (3 mth preceding over the 3-mth intervention period \& $3 \mathrm{mth}$ period
$\mathrm{P}-\mathrm{I})$

RCT

$\begin{array}{ll}\text { BPRS; SLOF; } & 36 \text { mth P-I: } \\ \text { (B, 1 wk, 18 } & 12 / 106 \\ \text { mth \& 36 mth } & (11.3) ; \\ \text { P-I) } & \text { FPGP: } \\ \text { No. \& duration } & 4 / 35(11.4) \text {; } \\ \text { of HA (6 mths } & \text { CG1: 3/36 } \\ \text { preceding } & (8.3) ; \text { CG2: } \\ \text { intervention, 1 } & 5 / 35(14.3) \\ \text { wk, 18 mth \& } & \\ \text { 36 mth P-I) } & \end{array}$

patients' functioning and no. and length of rehospitalisations in the SCMP group only sig improved at $15 \mathrm{mth} \mathrm{P}_{-}-$ timepoint.

$18 \mathrm{mth}$ P-I: There were sig Total: 6/96 6.3); 3/48 (6.3); 3/48 (6.3); (6.3)

differences between

MBPP and CG related to patients' insight into

illness, symptom

severity, functioning and number and length of rehospitalisation at $3 \mathrm{mth}$ and $18 \mathrm{mth}$ P-I. MBPP participants' symptom severity, illness insight and length of rehospitalisation improved sig at $3 \mathrm{mth}$ and $18 \mathrm{mth} \mathrm{P}-\mathrm{I}$ but functioning and number of rehospitalisation only sig improved $18 \mathrm{mth}$ P-I.

In the MSG there was a sig decrease in the duration of patient rehospitalisation at $3 \mathrm{mth}$ $\mathrm{P}$-I compared to the CG.

Functioning improved sig at $1 \mathrm{wk}$ and $18 \mathrm{mth}$ P-I. Re-hospitalisation of FPGP patients

decreased sig at the three respective time points in number and duration compared with CG1 and decreased sig at 1 wk and $18 \mathrm{mth} P$ CG2. In the FPGP there was a sig difference between patients SLOF score compared to PE \& 


\begin{tabular}{|c|c|c|c|}
\hline $\begin{array}{l}\text { Chien, } \\
\text { Thompson } \\
\text { \& Norman } \\
(2008)\end{array}$ & MSG: 38 & $\begin{array}{l}\text { Standard } \\
\text { care - } \\
\text { enhanced: } 38\end{array}$ & $\begin{array}{l}\text { Total: } 76 \\
\text { (32/44); } \\
\text { MSG: } 38 \\
\text { (17/21); } \\
\text { CG: } 38 \\
\text { (15/23) }\end{array}$ \\
\hline
\end{tabular}

Chien \& PE: 42
Wong

(2007)

\begin{tabular}{|c|}
\hline $\begin{array}{l}\text { Standard } \\
\text { care - } \\
\text { enhanced } 42\end{array}$ \\
\hline
\end{tabular}

28.8

enhanced: 42

DSM-IV

schizophrenia

diagnosis

$\begin{array}{ll}\text { Non-adapted } & \text { Total: } 42 \\ \text { SCST } & (34 / 8) ; \\ & \text { SCST: } 22 \\ & (16 / 6) ;\end{array}$

CG: $20(18 / 2)$

\section{DSM-IV SCID diagnosis}

SCST: 33.0; CG: 31.0

\section{(2013)}

Guo et al. CT: 633 (2010)

$\begin{array}{ll}\text { Medication: } & \text { Total: } 1268 \\ 635 & (698 / 570) \\ & \text { CT: } 633 \\ & (344 / 289) \\ & \text { CG: } 635 \\ & (354 / 281)\end{array}$

$\begin{array}{ll}\text { CT: } 26.1 ; & \text { DSM-IV SCID I } \\ \text { CG: } 26.4 & \text { schizophrenia } \\ & \text { /schizophreniform } \\ & \text { diagnosis rated } \\ & \text { by investigators } \\ & \text { or trained staff; } \\ & \text { PANSS total } \\ & \text { score }<60\end{array}$

$\begin{array}{ll}\text { MSG: } 2.7 ; & \text { Two } \\ \text { CG: } 2.6 & \text { psychiatric } \\ & \text { outpatient } \\ & \text { clinics, Hong } \\ & \text { Kong }\end{array}$

Two

psychiatric

clinics, Hong

Kong

RCT

\section{BPRS (B, 1 \\ wk \& $12 \mathrm{mth}$ \\ P-I) \\ No.\& duration \\ of HA (6 mths \\ preceding \\ intervention, 1}

P-I)

RCT (B, 1 wk \& 12 mth P-I) of HA (6 mths preceding intervention, wk \& $12 \mathrm{mth}$ P-I)

$\begin{array}{ll}\text { SCST: 11.8; } & \text { Outpatient } \\ \text { CG: } 8.4 & \text { clinic of the } \\ & \text { psychiatry } \\ & \text { and addiction } \\ & \text { hospital of } \\ & \text { Kasr Al-Ainy } \\ & \text { hospitals, } \\ & \text { Cairo } \\ & \text { university, } \\ & \text { Egypt }\end{array}$

RCT

PANSS

MSCEIT;

Part A and

Digit Symbol

Substitution

Test; The Digit

span task from

the Wechsler

Memory Scale;

The Proteus

Mazes task;

(B, P-I)

$\begin{array}{ll}\text { CT: } 2.1 ; & \text { Ten clinical } \\ \text { CG: } 2 & \text { sites in China } \\ & \text { (six university } \\ & \text { clinics } \\ & \text { and four } \\ & \text { province } \\ & \text { mental health } \\ & \text { agencies). }\end{array}$

\section{PANSS; ITAQ,}

GAS, ADL,

GAS $36(B ; 3$

I)

Rates of
No. \& duration

CG.

MSG the duration of patients

rehospitalisation

decreased sig at $12 \mathrm{mth}$ P-I.
12 mths $\mathrm{P}$ - In the PE group number I: Total: of patients
7/84 (8.3); rehospitalisation
PE: $3 / 42 \quad$ improved sig at 1 wk and
(7.1); CG: 12 mth P-I. However
4/42 (9.5) patients' functioning and
length of
rehospitalisation only sig improved at $12 \mathrm{mth}$ P-I.

\section{NR - but}

tables

suggest no

attrition

\section{SCST showed sig}

improvements in social

cognition compared to

CG. On the MSCEIT, the

SCST group showed sig improvements on the

total score and Branches

1 (Emotion perception)

and 4 (Managing

emotions)

Total:

Risk of relapse and

readmission was lower

mth \& 6 mth P- (41.3); CT: ITAQ scores was greate

227/633

ITAQ scores was greater

in the CT group than
CG. GAS and ADL

treatment 338/635

scores were also greater

over time for CT. CT

also scored higher on 4

domains of the SF-36

(role-physical, general

health, vitality, and roleemotional.) 


\section{SUPPLEMENTARY}

Habib et al. CaCBTp: 21 (2015)

Koolaee \& BFM: 21

PE:21

(2010)

BE: 21

Standard

care -

Total: 55

enhanced: 20

Kopelowicz ST: 45

et al. (2003)

$\begin{array}{ll}\text { Standard } & \text { Total: } 92 \\ \text { care: } 47 & (62 / 30) ; \\ & \text { ST: } 45 \\ & (30 / 15) ; \\ & \text { CG: } 47 \\ & (32 / 15)\end{array}$

Kopelowicz MFG-A: 64

Standard
care: 60

CG. 57

$(35 / 22)$

Total: 17

(114/60);

(43/21);

MFG-S: 53

(36/17);

$\begin{array}{lllll}\text { DSM-IV-TR } & \text { CaCBTp: } 8.8 ; & \begin{array}{l}\text { Psychiatric } \\ \text { inpatient }\end{array} & \text { RCT } & \text { PANSS; } \\ \text { inpatient } & \text { CG: } 8.6 & & \text { PSYRATS; } \\ \text { diagnosis } & & \text { clinic, Lahore } & & \text { SAI (B, P-I) } \\ \text { schizophrenia } & & \text { Pakistan } & \end{array}$

CaCBTp:

33.5;

CaCBTp:

G: 30.2

schizophrenia

Pakistan

DSM-IV

schizophrenia

diagnosis

3 yrs or less

Psychiatric

outpatien

clinic in

Tehran, Iran

ST: 37.6 ;

DSM-IV

schizophrenia/

schizoaffective

diagnosis; $\geq 1$

episode of

treatment in an

inpatient facility

$\geq 1$ wk duration in

previous $12 \mathrm{mths}$

NR

Community

mental health

centre, Los

Angeles

RCT

gen

QOLI; ROMI;

MMM +

generalisation

\section{$\mathrm{SMM}+$}

generalisation

assessments

(B, P-I \& 6 mth

P-I)

Medication

adherence

(monthly)

$\begin{array}{ll}\text { MFG-A: } 32.6 ; & \text { DSM-IV } \\ \text { MFG-S: 29.6 } & \text { structured clinical } \\ \text { CG: } 32.8 & \text { interview } \\ & \text { schizophrenia/ } \\ & \text { schizoaffective } \\ & \text { diagnosis }\end{array}$

\section{NR}

NR
Two

(Wommunity

mental health

Angeles
RCT

$\begin{array}{ll}\text { BPRS (B, P-I } & 12 \text { mth P-I? } \\ \text { \& 12-mth P-I; } & \text { Total: } \\ \text { medication } & 33 / 178 \\ \text { compliance (B, } & (18.4) \\ \text { 4 mth \& } 8 \text { mth } & \text { MFG-A: } \\ \text { P-B, P-I, 6 mth } & 10 / 64 \\ \& 12 \text { mth P-I) } & (15.6) ; \\ \text { HA rates (4 } & \text { MFG-S: }\end{array}$

CaCBTp group

demonstrated sig greater

improvement on PANSS

pos, neg and general

symptoms and

PSYRATS

hallucinations, delusions

and insight $\mathrm{P}-\mathrm{I}$

PE and BFM groups

exhibited sig greate

reduction in pos

symptoms at 3 and 6

Total: 7

(11.3)

mths P-I compared to

$\begin{array}{ll}\mathrm{PE}: 2 / 21 & \text { CG. PE pos symptoms } \\ \text { (9.5) } & \text { reduced sig from } B \text { to } 6\end{array}$

CG: $2 / 20$ mths P-I when compared

to BFM. Hospitalisation

data NR.

6 mth P-I: ST participants showed

Total: sig reduced pos,

8/102 (7.8) negative and total

ST: $6 / 45$ symptoms post

(13.3); CG: intervention which were

2/47 (4.3) maintained at 6-mth P-I.

In the ST group there

was also a sig main

effect on skill acquisition

for medication and

symptom management

skills. ST showed sigly

improved level of

functioning compared to

CG at P-I. There was

also sig lower

rehospitalisation rates in

the ST group from B to

follow-up compared to the $C G$.

At P-I, MFG-A

demonstrated sig higher medication adherence than MFG-S or the CG.

The MFG-A participants had a longer time to first hospitalization and were less likely to be 


\begin{tabular}{|c|c|c|c|c|c|c|c|c|c|c|c|}
\hline $\begin{array}{l}\text { Kulhara et } \\
\text { al. (2009) }\end{array}$ & PE: 38 & $\begin{array}{l}\text { Standard } \\
\text { care - } \\
\text { enhanced: } 38\end{array}$ & $\begin{array}{l}\text { Total: } 76 \\
\text { (42/34); } \\
\text { PE: } 38 \\
(17 / 21) ; \\
\text { CG: } 38 \\
(25 / 13)\end{array}$ & $\begin{array}{l}\text { PE: } 31.1 \text {; } \\
\text { CG: } 31.6\end{array}$ & $\begin{array}{l}\text { DSM-IV } \\
\text { structured } \\
\text { interview } \\
\text { schizophrenia } \\
\text { diagnosis }\end{array}$ & $\begin{array}{l}\text { PE: } 4.7 \text {; } \\
\text { CG: } 5.1\end{array}$ & $\begin{array}{l}\text { Department of } \\
\text { psychiatry of } \\
\text { a tertiary-care } \\
\text { hospital in } \\
\text { north-India }\end{array}$ & $\mathrm{RCT}$ & $\begin{array}{l}\text { PANSS; } \\
\text { WHODAS (B, } \\
1,2,3,4,5,6 \\
\& 7 \text { mth P-B; } \\
\text { P-I) } \\
\text { Relapse (over } \\
\text { the } 9 \text { mth } \\
\text { intervention } \\
\text { period). }\end{array}$ & $\begin{array}{l}(16.7) \\
\text { P-I: } \\
\text { Total: } \\
33 / 76 \\
\text { (43.4) PE: } \\
15 / 38 \\
(39.5) \text {; CG: } \\
18 / 38 \\
(47.4) \text {. }\end{array}$ & $\begin{array}{l}\text { There was a sig greater } \\
\text { decline in monthly } \\
\text { PANSS scores in the PE } \\
\text { group on all three } \\
\text { subscales in comparison } \\
\text { to the CG group. The } \\
\text { only patient outcome } \\
\text { showing a sig difference } \\
\text { from B to P-I was } \\
\text { disability level, with the } \\
\text { PE group showing sig } \\
\text { lower levels of disability } \\
\text { at } P \text {-I than the CG } \\
\text { group. }\end{array}$ \\
\hline $\begin{array}{l}\text { Kumar et } \\
\text { al. (2010) }\end{array}$ & HMCT: 8 & $\begin{array}{l}\text { Standard } \\
\text { care: } 8\end{array}$ & $\begin{array}{l}\text { Total: } 16 / 0 \\
\text { HMCT: } 8 / 0 \text {; } \\
\text { CG: } 8 / 0\end{array}$ & $\begin{array}{l}\text { HMCT: } 31.5 ; \\
\text { CG: } 34.1\end{array}$ & $\begin{array}{l}\text { ICD-10 patient } \\
\text { diagnosis } \\
\text { paranoid } \\
\text { schizophrenia }\end{array}$ & $\begin{array}{l}\text { HMCT: } 7.6 \text {; } \\
\text { CG: } 6.5\end{array}$ & $\begin{array}{l}\text { Inpatient } \\
\text { hospital, } \\
\text { central } \\
\text { institute of } \\
\text { psychiatry, } \\
\text { Ranchi, India }\end{array}$ & $\mathrm{RCT}$ & $\begin{array}{l}\text { PANSS; (B, } 2 \\
\text { wk P-B, P-I) }\end{array}$ & NR & $\begin{array}{l}\text { The HMCT group } \\
\text { demonstrated a sig } \\
\text { greater reduction in } \\
\text { positive symptoms P-I } \\
\text { compared to CG. }\end{array}$ \\
\hline $\begin{array}{l}\text { Kung et al. } \\
(2012)\end{array}$ & $\mathrm{FP}$ & $\begin{array}{l}\text { 'Comparison } \\
\text { group'- no } \\
\text { details }\end{array}$ & $\begin{array}{l}\text { Total: } 12 \text {; } \\
\text { FP: } 9 \text {; } \\
\text { CG: } 3\end{array}$ & $\begin{array}{l}\text { MFPG: } 34.2 \text {; } \\
\text { CG: } 50.6\end{array}$ & $\begin{array}{l}\text { 'schizophrenic } \\
\text { form of disorder' }\end{array}$ & NR & $\begin{array}{l}\text { Psychiatric } \\
\text { outpatient } \\
\text { clinics }\end{array}$ & $\begin{array}{l}\text { Non- } \\
\text { RCT }\end{array}$ & $\begin{array}{l}\text { BPRS; SANS; } \\
\text { SLOF; WQOL) } \\
\text { (baseline, } 3 \\
\text { mth post- } \\
\text { baseline, post- } \\
\text { intervention; } 3 \\
\text { mth post- } \\
\text { intervention) }\end{array}$ & No attrition & $\begin{array}{l}\text { In the MFPG group } \\
\text { BPRS and SANS scores } \\
\text { showed sig improvement } \\
\text { at post-intervention and } \\
\text { 3mth post-intervention. } \\
\text { The SLOF score was in } \\
\text { the opposite direction } \\
\text { than expected which } \\
\text { was consistent with the } \\
\text { previous within group } \\
\text { baseline, } 3 \text { mth baseline, } \\
\text { post-intervention and } 3 \\
\text { mth post-intervention. }\end{array}$ \\
\hline $\begin{array}{l}\text { Lak et al. } \\
(2010)\end{array}$ & $\begin{array}{l}\text { CBCSM + } \\
\text { SGT: } 35 \\
\text { CBCSM: } 35\end{array}$ & $\begin{array}{l}\text { 'Placebo' } \\
\text { education \& } \\
\text { rehab } \\
\text { programme: } \\
36\end{array}$ & $\begin{array}{l}\text { Total } 106 \\
(53 / 53) ; \\
\text { CBCSM + } \\
\text { SGT: } 35 \\
(18 / 17) ; \\
\text { CBCSM: } 35 \\
(17 / 18) ; \\
\text { CG: } 36 \\
(18 / 18)\end{array}$ & $\begin{array}{l}\text { CBCSM + } \\
\text { SGT: } 38.3 \text {; } \\
\text { CBCSM: } \\
44.5 ; \\
\text { CG: } 43.2\end{array}$ & $\begin{array}{l}\text { ICD-10 } \\
\text { schizophrenia } \\
\text { diagnosis, free } \\
\text { from positive } \\
\text { symptoms as } \\
\text { indicated by } \\
\text { (BPRS score > } \\
\text { 72) }\end{array}$ & $\begin{array}{l}\text { CBCSM + SGT: } \\
15.6 ; \\
\text { CBCSM: } 18.3 \\
\text { CG: } 18.9\end{array}$ & NR & $\mathrm{RCT}$ & $\begin{array}{l}\text { BPRS; GAF; } \\
\text { VSSS; ASSEI; } \\
\text { PWI (B, P-I , } 3 \\
\text { mth \& } 6 \text { mth P- } \\
\text { I) }\end{array}$ & $\begin{array}{l}6 \text { mth P-I: } \\
\text { Total: } \\
\text { 10/106 } \\
\text { (9.4); } \\
\text { CBCSM + } \\
\text { SGT: } 4 / 35 \\
\text { (11.4); } \\
\text { CBCSM: } \\
\text { 6/35 (17.1); } \\
\text { CG: } 0 / 36\end{array}$ & $\begin{array}{l}\text { P-I, the CBCSM + SGT } \\
\text { and the CBCSM group } \\
\text { scored sig higher than } \\
\text { the CG in social skills. At } \\
6 \text { mth P-I, social skill of } \\
\text { CBCSM + SGT was } \\
\text { better than the CBCSM } \\
\text { group and the CG. }\end{array}$ \\
\hline
\end{tabular}

mth \& 8 mth P- $13 / 54 \quad$ hospitalized than those B, P-I, 6 mth \& (24.1); $10 / 60=$

(16.7)

WHODAS (B, Tota: , 2, 3, 4, 5, $6 \quad 33 / 76$

Relapse (over the $9 \mathrm{mth}$

(3/3);

the CG group. The

showing a sig difference disability level, with the $P E$ group showing sig at $P-I$ than the $C G$

group.

CT group institute of

psychiatry,

Ranchi, India Psychiatric RCT

SANS; (baseline, 3

mth post-

intervention) 


\begin{tabular}{|c|c|c|c|c|c|c|c|c|c|c|c|}
\hline $\begin{array}{l}\text { Li \& Arthur } \\
\text { (2005) }\end{array}$ & FE: 46 & $\begin{array}{l}\text { Standard } \\
\text { care: } 55\end{array}$ & $\begin{array}{l}\text { Total: } 101 \\
(43 / 58) ; \\
\text { FE: } 46 \\
(18 / 28) ; \\
\text { CG: } 55 \\
(25 / 30)\end{array}$ & NR & $\begin{array}{l}\text { CCMD-II-R } \\
\text { inpatient } \\
\text { diagnosis } \\
\text { schizophrenia }\end{array}$ & NR & $\begin{array}{l}\text { Non-acute } \\
\text { Inpatient } \\
\text { hospital, } \\
\text { Beijing, China }\end{array}$ & $\begin{array}{l}\text { Cluster } \\
\text { RCT }\end{array}$ & $\begin{array}{l}\text { BPRS; GAS; } \\
\text { KASI; NOSIE; } \\
\text { relapse rates; } \\
\text { medication } \\
\text { compliance (B, } \\
\text { P-I, } 3 \text { mth \& } 9 \\
\text { mth P-I) }\end{array}$ & $\begin{array}{l}9 \text { mth P-I: } \\
12 / 101 \\
(11.9) ; \mathrm{FE} \\
3 / 46(6.5) ; \\
\text { CG: } 9 / 55 \\
(16.4)\end{array}$ & $\begin{array}{l}\text { FE group showed sig } \\
\text { greater improvements in } \\
\text { symptom severity, } \\
\text { knowledge and overall } \\
\text { functioning at } 9 \text { mth P-I } \\
\text { compared to the CG. }\end{array}$ \\
\hline $\begin{array}{l}\text { Li et al. } \\
\text { (2015) }\end{array}$ & CBT: 96 & $\begin{array}{l}\text { Supportive } \\
\text { therapy (TE): } \\
96\end{array}$ & $\begin{array}{l}\text { Total: } 192 \\
\text { (72/120); } \\
\text { CBT: } 96 \\
\text { (32/64); } \\
\text { CG: } 96 \\
(40 / 56)\end{array}$ & $\begin{array}{l}\text { CBT: } 29.3 \text {; } \\
\text { CG: } 33.4\end{array}$ & $\begin{array}{l}\text { SCID DSM-IV } \\
\text { Axis I } \\
\text { schizophrenia by } \\
\text { research } \\
\text { psychiatrists }\end{array}$ & $\begin{array}{l}\text { CBT: } 7.6 ; \\
\text { CG: } 8.8\end{array}$ & $\begin{array}{l}\text { Three } \\
\text { specialised } \\
\text { psychiatric } \\
\text { hospitals, } \\
\text { Beijing }\end{array}$ & $\begin{array}{l}\text { Cluster } \\
\text { RCT }\end{array}$ & $\begin{array}{l}\text { PANSS; SAI; } \\
\text { PSP (B, 12, } \\
24,36,60 \& \\
84 \text { wk P-B) }\end{array}$ & $\begin{array}{l}84 \text { wk P-B: } \\
\text { Total: } \\
25 / 192 \\
(14.6) ; \\
\text { CBT: } 11 / 96 \\
\text { (11.5); CG: } \\
\text { 14/96 } \\
(14.6)\end{array}$ & $\begin{array}{l}\text { Both groups had sig } \\
\text { decrease in all PANSS } \\
\text { symptoms, SAI insight } \\
\text { and PSP social } \\
\text { functioning P-I ( } 24 \text { wks) } \\
\text { which were maintained } \\
\text { up to } 84 \text { wks P-B. } \\
\text { Compared with CG, CBT } \\
\text { showed sig greater } \\
\text { reductions in PANSS } \\
\text { total and pos symptoms } \\
\text { and PSP functioning } \\
\text { scores from } 36 \text { wk P-B. }\end{array}$ \\
\hline $\begin{array}{l}\text { Lin et al. } \\
\text { (2013) }\end{array}$ & IMR: 48 & $\begin{array}{l}\text { Standard } \\
\text { care: } 49\end{array}$ & $\begin{array}{l}\text { Total: } 97 \\
(62 / 35) ; \\
\text { IMR: } 48 \\
\text { (30/18); } \\
\text { CG: } 49 \\
(32 / 17)\end{array}$ & $\begin{array}{l}\text { IMR: } 35.3 \text {; } \\
\text { CG: } 35.2\end{array}$ & $\begin{array}{l}\text { DSM-IV - } \\
\text { schizophrenia/ } \\
\text { schizoaffective } \\
\text { diagnosis }\end{array}$ & $\begin{array}{l}\text { IMR: 11.8; } \\
\text { CG: } 11.2\end{array}$ & $\begin{array}{l}\text { Acute } \\
\text { inpatient ward } \\
\text { in two } \\
\text { hospitals in } \\
\text { Taiwan }\end{array}$ & $\mathrm{RCT}$ & $\begin{array}{l}\text { BPRS; KI; } \\
\text { DAI-30; SAI- } \\
\text { E;. (B, P-I \& } 1 \\
\text { mth P-I) }\end{array}$ & $\begin{array}{l}1 \text { mth P-I: } \\
\text { Total: } \\
14 / 97 \\
\text { (14.4); } \\
\text { IMR: } 4 / 48 \\
(8.3) ; C G \\
10 / 49 \\
(20.4)\end{array}$ & $\begin{array}{l}\text { The IMR group showed } \\
\text { sig greater } \\
\text { improvements at P-I and } \\
\text { 1-mth P-I than the CG in } \\
\text { BPRS neg symptoms, } \\
\text { insight, illness- } \\
\text { management knowledge } \\
\text { and attitudes toward } \\
\text { medication. }\end{array}$ \\
\hline $\begin{array}{l}\text { Lin et al. } \\
(2013)\end{array}$ & IMR & NA & $\begin{array}{l}\text { Total: } \\
\text { IMR: } 26 \\
(18 / 8)\end{array}$ & 36.38 & $\begin{array}{l}\text { DSM-IV } \\
\text { schizophrenia/ } \\
\text { schizoaffective } \\
\text { diagnosis }\end{array}$ & NR & $\begin{array}{l}\text { Six } \\
\text { psychiatric } \\
\text { acute wards } \\
\text { at a } \\
\text { psychiatric } \\
\text { hospital in the } \\
\text { department of } \\
\text { psychiatry, } \\
\text { Taiwan }\end{array}$ & $\begin{array}{l}\text { Non- } \\
\text { RCT }\end{array}$ & $\begin{array}{l}\text { BPRS; KFI; } \\
\text { DAI-30; SAI-E } \\
\text { (B, P-I) }\end{array}$ & NR & $\begin{array}{l}\text { Participants improved in } \\
\text { each psychopathology } \\
\text { domain P-I, however } \\
\text { only the change in the } \\
\text { affective domain was } \\
\text { sig. }\end{array}$ \\
\hline $\begin{array}{l}\text { Mann \& } \\
\text { Chong } \\
\text { (2004) }\end{array}$ & SCP: 25 & $\begin{array}{l}\text { Education } \\
\text { programme: } \\
25\end{array}$ & $\begin{array}{l}\text { Total: } 50 \\
(38 / 12)\end{array}$ & 44.4 & $\begin{array}{l}\text { DSM-IV - } \\
\text { schizophrenia } \\
\text { diagnosis with } \\
\text { active positive } \\
\text { psychotic }\end{array}$ & $\begin{array}{l}\text { Average length } \\
\text { of stay in } \\
\text { hospital: } 26.2 \\
\text { (range: } 12-68 \\
\text { mths) }\end{array}$ & $\begin{array}{l}\text { Inpatient } \\
\text { psychiatric } \\
\text { hospital, } \\
\text { Hong Kong }\end{array}$ & $\mathrm{RCT}$ & $\begin{array}{l}\text { PANSS \& LSP } \\
\text { (B, P-I \& } 1 \mathrm{mth} \\
\text { P-I) }\end{array}$ & $\mathrm{NR}$ & $\begin{array}{l}\text { At P-I there was a sig. } \\
\text { improvement between } \\
\text { SCP and CG in PANSS } \\
\text { scores. However this } \\
\text { returned back to }\end{array}$ \\
\hline
\end{tabular}


symptoms $\geq 1 \mathrm{yr}$ assessed by

psychiatrist

\begin{tabular}{|c|c|c|c|}
\hline $\begin{array}{l}\text { Mausbach } \\
\text { et al. } \\
(2008)+\end{array}$ & PEDAL: 21 & $\begin{array}{l}\text { CG1: FAST } \\
\text { (non-adapted } \\
\text { PEDAL): } 15 \\
\text { CG2: Support } \\
\text { group (TE): } \\
23\end{array}$ & $\begin{array}{l}\text { Total: } 59 \\
\text { (35/24); } \\
\text { PEDAL: } 21 \\
\text { (11/10); } \\
\text { CG1: } 15 \\
(10 / 5) ; \\
\text { CG2: } 23 \\
(14 / 9)\end{array}$ \\
\hline
\end{tabular}

PEDAL: 50.7; DSM-IV

$\begin{array}{ll}\text { CG1: } 47.4 ; & \text { diagnosis } \\ \text { CG2: } 47.3 & \text { schizophre }\end{array}$

CG2: 47.3

(39/18);

CG: 59

$(31 / 28)$

$\begin{array}{llll}\begin{array}{l}\text { Patterson } \\ \text { et al. }\end{array} & \text { PEDAL: } 21 & \text { Support } & \text { Total: } 29 \\ \text { (2005)+ } & & \text { group (TE): } 8 & (14 / 15) ; \\ & & \text { PEDAL: } 21 \\ & & (11 / 10) ;\end{array}$

CG: $8(3 / 5)$

\section{Psychiatric clinics, San} schizophrenia/ disorder schizoaffective or

psychotic mood

disorder

PEDAL: 23.5
CG1: 24.5
CG2: 23.8

Board \& care

(B\&C)

facilities, San

Diego country

and mental

health clinics

near the USA-

Mexico border

\section{ICD-10 RDC}

diagnosis

schizophrenia or

related disorde

$\begin{array}{ll}\text { CaCBTp: } 4.7 ; & \text { Psychiatric } \\ \text { CG:5.8 } & \text { outpatient } \\ & \text { clinics, } \\ & \text { Karachi }\end{array}$

Age of illness onset (yrs): PEDAL: 28.3; SG: 43.5

Block
RCT

PANSS:

U-I:

(B, P-1)

(16.9)
PEDAL:

3/21 (14.3);

CG1: $2 / 15$

(13.3);

CG2: $5 / 23$

(21.7)

.7)

$\begin{array}{lll}\text { RCT } & \text { PANSS; } & \text { P-I } \\ & \text { PSYRATS; } & \text { Total: } \\ \text { SAI (B, P-I) } & 14 / 116 \\ & & (12.1) ; \\ & \text { CaCBT: } \\ & 6 / 59 \\ & & (10.1) ; \text { CG: } \\ & & 8 / 57(14)\end{array}$

Cluster PANSS;

SSPA;; QWB

\& 12 mth P-I)

(23.8)

(23.8)

5/21 (23.8);

CG: No

attrition

NR

P-I:

Total:

22/326

(6.7); FIG:

$\begin{array}{ll}\text { CG1: } & \text { Total: } 326 \\ \text { Medication } & \text { (128/198); } \\ \text { treatment: } & \text { FIG: } 126 \\ 110 & (44 / 82) ; \\ & \text { CG1: } 103 \\ \text { CG2: No } & (48 / 55) ;\end{array}$

$\begin{array}{ll}\text { ICD-10 \& CCMD- } & \text { FIG: } 11.6 ; \\ 2-R & \text { CG1: } 10.6 \\ \text { schizophrenia } & \text { CG2: } 12.3 \\ \text { diagnosis } & \end{array}$
FIG: 43.5 ;
CG1: 42.4
diagnosis
Cluster PSE; GPISS;
SDSS;
medication
compliance;
relapse rate
(B, P-I)

CG1: 2/105

baseline level at $1 \mathrm{mth}$

P-I.

PEDAL group showed a

sig improvement on the

UPSA compared to

those in CG1 and CG2

groups. The PEDAL

group had sig higher

SSPA scores at P-I

compared with the

CG1but not the CG2.Th

PEDAL group also made

sig fewer medication

errors at $\mathrm{P}-\mathrm{I}$ than the

CG2 group.

At $\mathrm{P}-\mathrm{I}$ the $\mathrm{CaCBTp}$ group showed sig greater

improvements compared with the CG in PANSS

pos and neg symptoms

and general

psychopathology,

PSYRATS delusions and hallucinations and insight.

At 6 mth P-I the PEDAL

had a sig greater UPSA

score than the CG.

However no sig

differences were found

between the groups at 6 $\mathrm{mth}$ and $12 \mathrm{mth}$ P-I. At 12 mth P-I, the PEDAL

group performed sig

better than the CG. CG

also had sig lower

PANSS ratings than the PEDAL group at $12 \mathrm{mth}$ P-I.

At P-I, FIG treatment

higher than that

CG1 and CG2. There

was no ${ }^{2}$ 2. There

clinical outcomes 
intervention: CG2: 97

115

$(36 / 61)$

Razali et al. CMFT: $80 \quad$ BFT: $86 \quad$ NR

(2000)

NR

R

DSM-IV
diagnosis
schizophrenia

CMFT: 13.7 ;
CG:14.2

Outpatient
hospital,
University
Hospital
(USM) Kota
Bharu, West
Malaysia

Shin \&
Lukens
$(2002)$

Supportive
therapy (TE):
24

Total: 48
(20/28);
PE: 24
(7/17);
CG: 24
(13/11)

So et al.

MCTd: 23

$\begin{array}{ll}\text { Standard } & \text { Total: } 44 \\ \text { care }- & (24 / 20) ; \\ \text { medication } & \text { MCTd: } 23 \\ \text { consultations: } & (12 / 11) ; \\ 21 & \text { CG: } 21(12 / 9)\end{array}$
21 CG: $21(12 / 9)$

PE: 39.5 ;
CG: 34.7

$\begin{array}{ll}\text { DSM-IV } & \text { Number of } \\ \text { diagnosis } & \text { hospitalisations: } \\ \text { schizophrenia, } & \text { PE: } 2.7 ; \\ \text { schizoaffective, } & \text { CG: } 1.2 \\ \text { or } & \text { Time since last } \\ \text { shizophreniform } & \text { hospitalisation } \\ \text { disorder rated by } & \text { (mths): } \\ \text { psychiatrist } & \text { PE: } 7.2 ; \\ & \text { CG: } 12.7\end{array}$

Outpatient

mental health

clinic, Queens

$\begin{array}{llll}\text { MCTd: } 32.4 ; & \text { Casenote } & \text { Number of } & \text { Outpatient } \\ \text { CG: } 35.6 & \text { diagnosis } & \text { hospitalisations: } & \text { clinic, Hong } \\ & \text { schizophrenia, } \geq 4 & \text { MCTd: } 1.6 ; & \text { Kong } \\ & \text { PANSS delusions } & \text { CG: } 0.9 & \end{array}$

items

$\begin{array}{ll}\text { Standard } & \text { Total: } 82 \\ \text { care - } & (64 / 18) ; \\ \text { medication } & \text { PSST: 43 } \\ \text { consultations: } & \text { (31/12); } \\ 49 & \text { CG: } 39(33 / 6)\end{array}$

RCT

RCT

$\mathrm{RCT}$

$\begin{array}{ll}\text { PSST: } 29.7 ; & \text { DSM-IV } \\ \text { CG: } 30.1 & \& \text { CIDI } \\ & \text { schizophrenia } \\ & \text { diagnosis with } \\ & \text { clinically stable } \\ & \text { psychotic } \\ & \text { symptoms }(\geq 60 \\ & \text { PANSS score })\end{array}$

$\begin{array}{ll}\text { Age of illness } & \text { Psychiatric } \\ \text { onset (yrs): } & \text { outpatient } \\ \text { PSST: } 21.3 ; & \text { hospital, } \\ \text { CG: } 21.2 & \text { Mexico }\end{array}$

$\mathrm{RCT}$
Valencia et PSST: 49 Valencia
al. (2007)
(1.9); CG2: $18 / 115$

$$
\text { (15.7) }
$$

BPRS; GAF;
SBS; relapse
rates; HA
rates;
medication
compliance (B
6 mth \& 12
mth P-B)

BPRS; SDS
(B, P-I)

6 \& $12 \mathrm{mth}$

P-B

Total:

23/166

(13.9)

$6 / 80(7.5)$;

CG: $17 / 86$

(19.8)

PANSS;
PSYRATS;
(B, P-I \& 1
mth P-I) WAIS
(B or pre-
intervention
only)

NR (tables

At P-I, the PE group attrition)

reduced symptom

severity and perception

of stigma in comparison

to the CG group.

FIG group and

while the

recovery and sig

improvement in FIG \&

CG1 was sig higher than

that in the CG2. The

lower than CG1.

mth P-B the CMF group showed sig

variables compared to the CG.

1 mth P-I:

Total:

$18 / 44$

(40.9)

MCTd:

10/23

(43.5); CG: PSYRATS delusions and

At P-I there was a large effect size of

improvement for the

MCTd group's PANSS

pos scores, PANSS

delusions score,

PSYRATS delusional

conviction.

PANSS; P-I:

PSFS; GAF Total:

$\begin{array}{ll}\text { (B, P-I) } & 16 / 98 \\ \text { Relapse rates; } & (16.3)\end{array}$

HA rates; PSST: 6/49

At P-I, the PSST group showed sig improved symptomatology scores on total score, pos and PSST. 6/49 neg symptoms and GPS (12.2); CG: compared with the CG,

compliance; $\quad 10 / 49 \quad$ with large effect sizes fo therapeutic (20.4) adherence both groups. Sig.

improvements were also observed in the PSST 
intervention

period)

\begin{tabular}{|c|c|c|c|c|c|c|c|}
\hline $\begin{array}{l}\text { Valencia et } \\
\text { al. (2010) }\end{array}$ & PSST: 54 & $\begin{array}{l}\text { Standard } \\
\text { care - } \\
\text { medication } \\
\text { consultations: } \\
53\end{array}$ & $\begin{array}{l}\text { Total: } 83 \\
\text { (60/23); } \\
\text { PSST: } 47 \\
\text { (34/13); } \\
\text { CG: } 36 \\
(26 / 10)\end{array}$ & $\begin{array}{l}\text { PSST: 29.9; } \\
\text { CG: } 29.5\end{array}$ & $\begin{array}{l}\text { DSM-IV } \\
\text { \& CIDI } \\
\text { schizophrenia } \\
\text { diagnosis }\end{array}$ & $\begin{array}{l}\text { Age of illness } \\
\text { onset (yrs) } \\
\text { PSST: } 22.4 \text {; } \\
\text { CG: } 21.1\end{array}$ & $\begin{array}{l}\text { National } \\
\text { institute of } \\
\text { psychiatry } \\
\text { outpatient } \\
\text { clinic, Mexic } \\
\text { city }\end{array}$ \\
\hline $\begin{array}{l}\text { Wahass \& } \\
\text { Kent (1997) }\end{array}$ & MPI: 3 & $\begin{array}{l}\text { Standard } \\
\text { care - } \\
\text { medication } \\
\text { consultations: } \\
3\end{array}$ & $\begin{array}{l}\text { Total: } 6(6 / 0) \text {; } \\
\text { MPI: } 3(3 / 0) \\
\text { CG: } 3(3 / 0)\end{array}$ & $\begin{array}{l}\text { MPI: } 31.3 \text {; } \\
\text { CG: } 34.0\end{array}$ & $\begin{array}{l}\text { ICD-10 } \\
\text { schizophrenia } \\
\text { diagnosis }\end{array}$ & $\begin{array}{l}\text { Experiencing } \\
\text { persistent } \\
\text { auditory } \\
\text { hallucinations } \\
\text { for at least } 4 \text { yrs }\end{array}$ & NR \\
\hline $\begin{array}{l}\text { Wang et al. } \\
(2013)\end{array}$ & SCIT: 22 & $\begin{array}{l}\text { Standard } \\
\text { care - } \\
\text { medication } \\
\text { consultations: } \\
17\end{array}$ & $\begin{array}{l}\text { Total: } 39 \\
\text { (20/19); } \\
\text { SCIT: } 22 \\
\text { (12/10); } \\
\text { CG: } 17(8 / 9)\end{array}$ & $\begin{array}{l}\text { SCIT: } 43.9 \text {; } \\
\text { CG: } 40.9\end{array}$ & $\begin{array}{l}\text { DSM-IV } \\
\text { schizophrenia } \\
\text { diagnosis }\end{array}$ & NR & NR \\
\hline $\begin{array}{l}\text { Weng et al. } \\
(2005)\end{array}$ & MRP: 62 & $\begin{array}{l}\text { Standard } \\
\text { care - }\end{array}$ & $\begin{array}{l}\text { Total: 124; } \\
\text { MRP: NR; }\end{array}$ & NR & $\begin{array}{l}\text { DSM-III-R } \\
\text { schizophrenia }\end{array}$ & NR & $\begin{array}{l}\text { An-ding } \\
\text { psychiatric }\end{array}$ \\
\hline
\end{tabular}

group for all areas of

psychosocial functioning

and in the GPFS

compared with the CG

and also with global

functioning. During the

intervention the PSST

group had a sig higher

level of compliance with

medication, therapeutic

adherence and sig lower

relapse rate than the

CG.

RCT

PANSS:
(B, P-I)
Relapse

Relapse

adherence

adherence to

medication
(over 12 mth

intervention

period)

RCT

SAHI; $10 \mathrm{~cm}$
VAS to

measure

hallucination

severity; Two

VAS to

measure

anxiety and

anxiety and

depression in

response to
voices $(B, P-I$,

3 mth $P-I)$

RCT PANSS;

WAIS; PSP:

FEIT;

Computerised

version of the

Eyes Task;

Attributiona

Style

Questionnaire

(B, 6 mth P-I)

RCT
BPRS; NOSIE;
SDSS; relapse
P-I:

Total:

NR -

tables

psychiatric
$24 / 108$

PSST: 7/54

(13); CG

$17 / 54$

(31.5)

suggest no

attrition

At P-I, the PSST group exhibited sig improved symptomatology,

adherence to

medication, attendance

at appointments, social

functioning, relapse and

rehospitalisation rates.

\section{Symptom ratings}

improved for two

participants in the MPI

group but there were no

changes in the third

participant. The third

patient also reported that

the loudness of his

voices increased over

time.

6 mth P-I:

Total: $4 / 43$

(9.3) SCIT:

$0 / 22(0)$;

(19)

The SCIT group showed

a sig improvement in

emotion perception,

theory of mind,

attributional style and

social functioning

compared with the CG.

12 mth P-I: Total:

BPRS, NOSIE, SDSS relapse and 
diagnosis

hospital

ward, Beijing

Xiang et al. PFI: 36

\section{Medication}

treatment:

Total: 77

PFI: 40.5;

CG: 41

CG: 41.2

Schizophrenia \&

affective

psychoses
15.3

Xinjin county,

China

Psychiatric outpatient clinic \& home visits

\section{5} assessed on admission

medication

treatment: 29

31

$\begin{array}{lll}\text { Standard } & \text { Total: } 3,082 & \text { FE: } 40.3 ; \\ \text { care: } 1016 & (1,821 / 1,261) ; & \text { CG: } 40.8 \\ & \text { FE: } 2,076 & \\ & (1,239 / 837) ; & \\ & \text { CG: } 1,016 & \\ & (582 / 424) & \end{array}$

CCMD-2

schizophrenia

diagnosis
FE: 15.1;

CG: 15.5

Heqin
$(1993)^{\wedge}$
FE: 2076
Zhang et

FIG: 42
Standard

care: 41
FIG: 23.5;

CG: 24.1
\& HA rates

(every 2 wks

during

intervention;

monthly $\mathrm{P}-\mathrm{I}$ up

to $12 \mathrm{P}-\mathrm{I})$

RCT

PSE; SDSS
med
compliance
rates (B, P-I

compliance
rates $(\mathrm{B}, \mathrm{P}-\mathrm{I}$

NR

MRP: $1 / 62$

(1.61): $\mathrm{CG}$ :

$1 / 62(1.61)$

group from $B$ to $P$-I and

NR

Med compliance sig

higher in the PFI group

than in the CG P-I, in

addition the total rate of

improvement in clinical

state and level of social

functioning was sig

higher in the PFI group
than in the CG group P-I.

RCT

BPRS; SAPS; SANS; GAF;

SDSS; no. \&

duration of $\mathrm{HA}$;

relapse rates;

meds

compliance $(\mathrm{B}$,

$6,12 \& 18$

mths P-I)

18 mths P-

I: Total:

$4 / 63(6.3)$

(5.9)

CG: $2 / 29$

tudy-specific

questionnaire;

symptoms,

recovery,

relapse \&

hospitalisation;

WHO- DAS (B,

6 \& 12 mth P.

l)

(6.9)

)

\section{Duration of}

rehospitalisation sig

shorter for FIG group at

12 and $18 \mathrm{mths}$, and

fewer relapsed at 12

mths than CG. BPRS,

GAF and SDSS scores

at $12 \mathrm{mths}$ and SAPS,

BPRS, GAF and SDSS

scores at 18 mths

showed sig greater

improvements in FIG

group.

At P-I, participants in the

FE group showed a

higher rate of recovery

or stabilization of

condition; a greater

reduction in

exacerbation of both pos

and neg symptoms,

relapse rates, number

institutionalized, duration

of hospitalization, plus

total and factor score on

the DAS.
Psychiatric

outpatient

clinic and

family home

$\begin{array}{ll}\text { RCT } & \text { BPRS; GAS; } \\ & \text { HA rates } \\ & \text { (baseline; P-I, } \\ & 3,6,9,12,15 \\ & \& 18 \text { mth P-I) }\end{array}$

association

admission

patients with a

\section{$18 \mathrm{mth}$}

post-

At $18 \mathrm{mth}$ P-I,

participants in the $\mathrm{FI}$

group who were not

$\begin{array}{ll}\text { Total: 5/83 } & \text { readmitted had less } \\ \text { (6); FIG: } & \text { severe psychiatric }\end{array}$ 


\section{SUPPLEMENTARY}

diagnosis of

schizophrenia

$\begin{array}{llll}\begin{array}{l}\text { Zhang et al. } \\ (1998) \wedge\end{array} & \text { FE: } 682 & \text { Standard } & \text { Total: } 1037 \\ & & \text { care: } 355 & (642 / 395) ; \\ & & \text { FE: } 682 \\ & & (420 / 262) \\ & & \text { CG: } 355\end{array}$

(222/133)

$\begin{array}{llll}\text { Zimmer et } & \text { IPT: } 23 & \text { Standard } & \text { Total: } 56 \\ \text { al. (2007) } & & \text { care }- & (42 / 14) ; \\ & \text { medication } & \text { IPT: } 20 \\ & \text { consultation: } & (17 / 3) ; \\ & 43 & \text { CG: } 36 \\ & & (25 / 11)\end{array}$

CG: 39.0

CCMD-2

schizophrenia

FE: 7.3 ;
CG: 7.7

diagnosis

\begin{abstract}
IPT: 36.1 ;
CG: 39.3
\end{abstract}

$\begin{array}{ll}\text { 'Schizophrenia } & \text { IPT: } 15.3 \text {; } \\ \text { outpatient } & \text { CG: } 17.1 \\ \text { database' } & \end{array}$

\begin{tabular}{|c|c|c|c|}
\hline & & & $\begin{array}{l}3 / 42(7.1) \\
\text { CG: } 2 / 41 \\
(4.9)\end{array}$ \\
\hline NR & $\begin{array}{l}\text { Cluster } \\
\text { RCT }\end{array}$ & $\begin{array}{l}\text { Study-specific } \\
\text { questionnaire; } \\
\text { to assess } \\
\text { severity of the } \\
\text { illness and } \\
\text { symptoms, } \\
\text { relapse, } \\
\text { duration of HA; } \\
\text { WHO-DAS (B, } \\
6,12 \& 24 \text { mth } \\
\text { P-B) }\end{array}$ & $\begin{array}{l}24 \text { mth P-B } \\
\text { Total: } 236 / \\
1284(18.4) \\
\text { FE: } \\
\text { 169/851 } \\
(19.9) ; \\
\text { CG: } 67 / 433 \\
(15.5) .\end{array}$ \\
\hline $\begin{array}{l}\text { Schizophrenia } \\
\text { outpatient } \\
\text { program of } \\
\text { the hospital } \\
\text { de clinicas de } \\
\text { porto alegre } \\
\text { (HCPA) }\end{array}$ & $\mathrm{RCT}$ & $\begin{array}{l}\text { MMSE; GAF; } \\
\text { SOFAS; } \\
\text { WHOQOL- } \\
\text { BREF; SAS } \\
\text { (B, P-I) }\end{array}$ & $\begin{array}{l}\text { Total: } 4 / 56 \\
\text { (7.1) NR } \\
\text { by group }\end{array}$ \\
\hline
\end{tabular}

CG: $2 / 41$

oning and highe

overall functioning

compared to members of

the CG who were not

readmitted. The

proportion of patients

who were readmitted to

hospital was sig greater

among those in the CG

in comparison to

participants who

received the $\mathrm{FI}$.

At $24 \mathrm{mth} P-B$, those in

the FE group showed a

higher rate of recovery

and stabilisation of the

condition; a lower rate of exacerbation of

symptoms and a

reduction in annual

relapse rates and annual

times institutionalised in

comparison to the $\mathrm{CG}$.

At P-I, the IPT group

demonstrated sig

improved scores on

cognition in the domains

of spatiotemporal

orientation, memory,

social adjustment,

leisure/social life, family

relations, overall

functioning, social

occupational functioning

and quality of life in the

psychological domain

\footnotetext{
Note:

* Same sample - Chien, Chan \& Thompson. (2006) reports 18 follow-up data for participants in Chien \& Chan (2004)

^ Same sample - Zhang et al. (1998) reports 24 month follow-up for subsample of participants (2/5 catchment areas) in Zhang \& Hequin (1993)

Standard care $=$ usual care package with case management/care coordinator and psychiatrist visits to monitor medication
}

+ Same sample - Mausbach et al. (2008) uses same participants as Patterson et al. (2005) in addition to participants from a second wave of recruitment (SG: $n=15$; FAST: $n=15$ ) 


\section{SUPPLEMENTARY}

Standard care enhanced $=$ offered additional education or counselling to usual case management intervention.

Timepoints: $\mathrm{B}$ = baseline; $\mathrm{P}-\mathrm{I}=$ post-intervention; $\mathrm{P}-\mathrm{B}=$ post-baseline; $\mathrm{RA}=$ research assistant; $\mathrm{HA}=$ hospital admission; $T E=$ time equivalent

Bold text $=$ included in meta-analysis on symptoms

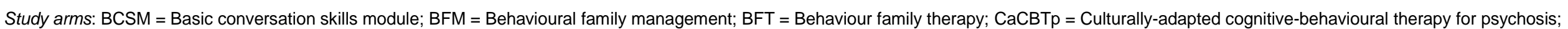

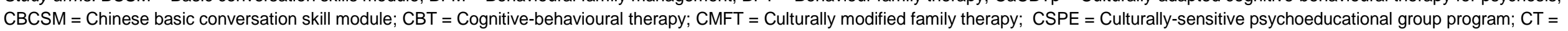

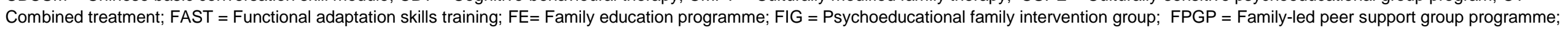

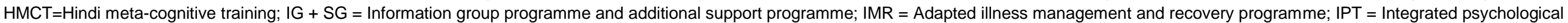

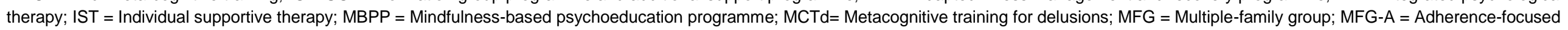

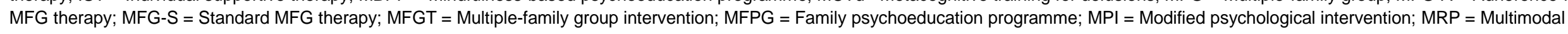

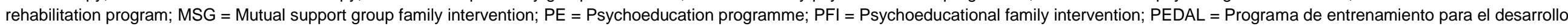

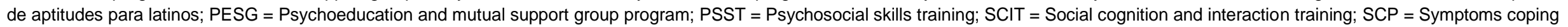

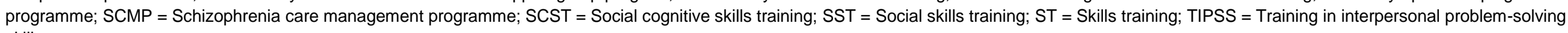
skills

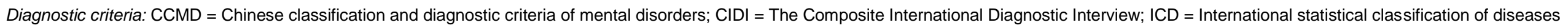

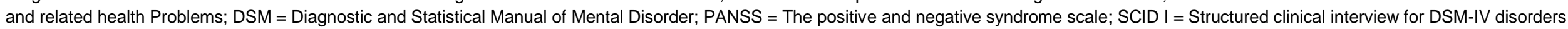

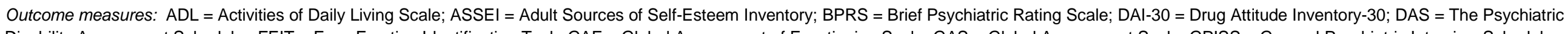

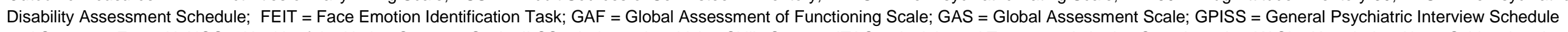

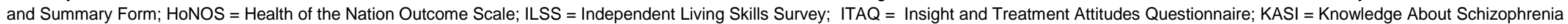

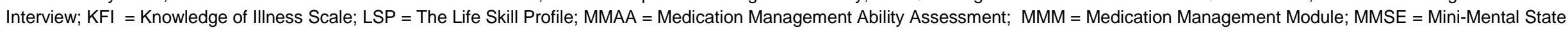

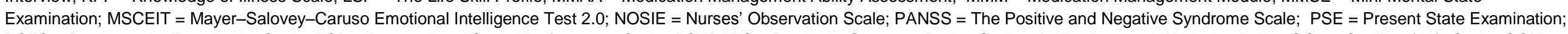

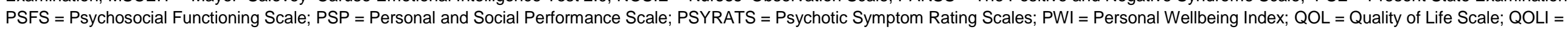

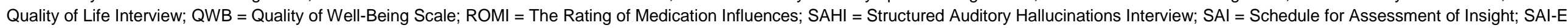

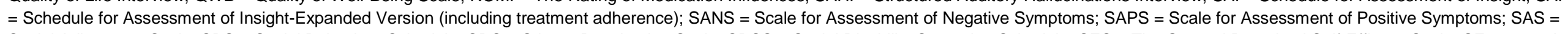

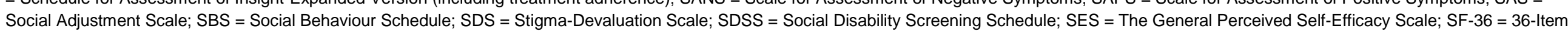

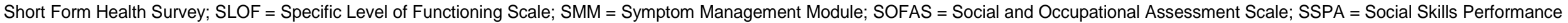

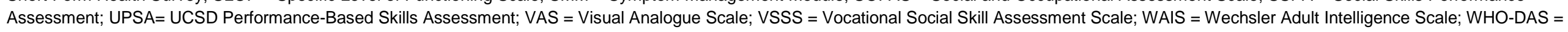

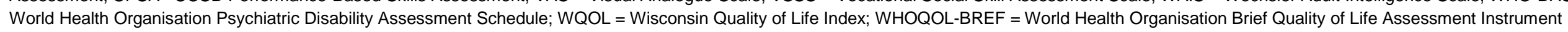




\section{SUPPLEMENTARY}

\section{Appendix 5}

Table A5. Characteristics of interventions included in the systematic review of culturally-adapted psychosocial interventions for psychosis $(n=46)$

\begin{tabular}{|c|c|c|c|c|c|c|c|c|c|c|c|}
\hline \multirow[b]{2}{*}{$\begin{array}{l}\text { Author } \\
\text { (year) }\end{array}$} & \multicolumn{3}{|c|}{ Target population } & \multicolumn{2}{|c|}{ Intervention model } & \multicolumn{6}{|c|}{ Intervention delivery } \\
\hline & Country & $\begin{array}{l}\text { Adapted } \\
\text { for } \\
\text { (minority/ } \\
\text { majority) }\end{array}$ & Subculture & $\begin{array}{l}\text { Adapted from } \\
\text { (western } \\
\text { model/theory) }\end{array}$ & Intervention type & $\begin{array}{l}\text { Intervention } \\
\text { attendees }\end{array}$ & $\begin{array}{l}\text { Modality } \\
\text { (individual/ } \\
\text { group) }\end{array}$ & $\begin{array}{l}\text { Number of } \\
\text { sessions, } \\
\text { frequency, \& } \\
\text { duration }\end{array}$ & $\begin{array}{l}\text { Duration of } \\
\text { intervention }\end{array}$ & $\begin{array}{l}\text { Therapy } \\
\text { setting }\end{array}$ & Therapist training \\
\hline $\begin{array}{l}\text { Bradley et } \\
\text { al. (2006) }\end{array}$ & Australia & Minority & Vietnamese & $\begin{array}{l}\text { Multiple-family } \\
\text { psychoeducational } \\
\text { group treatment } \\
\text { manual (McFarlane } \\
\text { et al., 1991) }\end{array}$ & $\begin{array}{l}\text { Family } \\
\text { intervention } \\
\text { (MFGT) }\end{array}$ & $\begin{array}{l}\text { Caregivers } \\
\text { and patients }\end{array}$ & Group & $\begin{array}{l}26 \text { sessions, } \\
\text { every } 2 \text { wks }\end{array}$ & 12 mths & $\begin{array}{l}\text { Clinical + } \\
\text { community }\end{array}$ & $\begin{array}{l}\text { Primary therapists \& } \\
\text { support facilitators. } \\
\text { Training initially } \\
\text { provided by a three- } \\
\text { day national } \\
\text { workshop conducted } \\
\text { by William } \\
\text { McFarlane }\end{array}$ \\
\hline $\begin{array}{l}\text { Carrà et al } \\
(2006)\end{array}$ & Italy & Majority & Italian & $\begin{array}{l}\text { Psychoeducational } \\
\text { multifamily groups } \\
\text { (McFarlane et al. } \\
\text { 2002) }\end{array}$ & $\begin{array}{l}\text { Family } \\
\text { intervention (IG; } \\
I G+S G)\end{array}$ & Caregivers & Group & $\begin{array}{l}\text { IG: } \\
24 \text { wkly } \\
\text { meetings } \\
\text { (1.75 hrs } \\
\text { duration); } \\
\text { SG: } \\
48 \text { wkly } \\
\text { sessions (1.5 } \\
\text { hrs duration) }\end{array}$ & 24 mths & Community & $\begin{array}{l}\text { Two specifically } \\
\text { trained psychiatrists } \\
\text { not involved in } \\
\text { patients' community } \\
\text { standard care }\end{array}$ \\
\hline $\begin{array}{l}\text { Chan et al. } \\
\text { (2009) }\end{array}$ & China & Majority & Chinese & $\begin{array}{l}\text { Care management } \\
\text { in early psychosis } \\
\text { handbook (Early } \\
\text { psychosis } \\
\text { prevention and } \\
\text { intervention centre, } \\
2001 \text { ) }\end{array}$ & $\begin{array}{l}\text { Family } \\
\text { intervention } \\
(\mathrm{PE})\end{array}$ & $\begin{array}{l}\text { Family } \\
\text { caregivers } \\
\text { and patients }\end{array}$ & Group & $\begin{array}{l}10 \text { wkly } \\
\text { sessions }\end{array}$ & $3 \mathrm{mths}$ & Clinical & $\begin{array}{l}\text { A mental health } \\
\text { nurse with more than } \\
15 \text { years of working } \\
\text { experience in } \\
\text { community mental } \\
\text { health setting. }\end{array}$ \\
\hline $\begin{array}{l}\text { Chien } \\
\text { (2008) }\end{array}$ & China & Majority & Chinese & $\begin{array}{l}\text { Psychoeducation: } \\
\text { family } \\
\text { psychoeducational }\end{array}$ & $\begin{array}{l}\text { Family } \\
\text { intervention } \\
\text { (PESG) }\end{array}$ & $\begin{array}{l}\text { Primary } \\
\text { caregiver, } \\
\text { patient and }\end{array}$ & Group & $\begin{array}{l}18 \text { sessions } \\
\text { ( } 2 \text { hrs } \\
\text { duration) }\end{array}$ & $9 \mathrm{mths}$ & Clinical & $\begin{array}{l}\text { Research psychiatric } \\
\text { nurse, psychiatrist, } \\
\text { research nurse, }\end{array}$ \\
\hline
\end{tabular}




\section{SUPPLEMENTARY}

\begin{tabular}{|c|c|c|c|c|c|c|c|c|c|c|c|}
\hline & & & & $\begin{array}{l}\text { support groups } \\
\text { (Posner, 1992); } \\
\text { Mutual support: } \\
\text { support groups } \\
\text { theory \& practise } \\
\text { (Galinsky \& } \\
\text { Schopler,1995) \& } \\
\text { how to work with } \\
\text { self-help group } \\
\text { guidelines (Wilson, } \\
\text { 1995) }\end{array}$ & & $\begin{array}{l}\text { other family } \\
\text { members }\end{array}$ & & & & & $\begin{array}{l}\text { clinical psychologist } \\
\& \text { nurse researcher }\end{array}$ \\
\hline $\begin{array}{l}\text { Chien \& } \\
\text { Chan } \\
(2004)^{*}\end{array}$ & China & Majority & Chinese & $\begin{array}{l}\text { Psychoeducation: } \\
\text { Multiple-family } \\
\text { groups and } \\
\text { psychoeducation } \\
\text { (McFarlane et al. } \\
\text { 1995) } \\
\text { Mutual support: } \\
\text { Family intervention } \\
\text { for Asian Americans } \\
\text { (Bae \& Kung, 2000) }\end{array}$ & $\begin{array}{l}\text { Family } \\
\text { intervention } \\
\text { (MSG; PE) }\end{array}$ & $\begin{array}{l}\text { Family } \\
\text { caregivers } \\
\text { and patients }\end{array}$ & Group & $\begin{array}{l}\text { MSG: } \\
12 \text { sessions } \\
\text { every } 2 \text { wks } \\
\text { (2 hrs } \\
\text { duration); } \\
\text { PE: } 12 \\
\text { sessions bi } \\
\text { wkly (2 hrs } \\
\text { duration) }\end{array}$ & $6 \mathrm{mths}$ & Clinical & $\begin{array}{l}\text { MSG: peer leader } \\
\text { trained by } \\
\text { researchers; } \\
\text { PE: Two psychiatric } \\
\text { nurses experienced } \\
\text { in psychiatric } \\
\text { rehabilitation } \\
\text { programs. }\end{array}$ \\
\hline $\begin{array}{l}\text { Chien \& } \\
\text { Chan } \\
(2013)^{\star}\end{array}$ & China & Majority & Chinese & $\begin{array}{l}\text { Psychoeducation: } \\
\text { Multiple-family } \\
\text { groups and } \\
\text { psychoeducation } \\
\text { (McFarlane et al. } \\
\text { 1995); } \\
\text { Mutual support: } \\
\text { Family intervention } \\
\text { for Asian Americans } \\
\text { (Bae \& Kung, } \\
\text { 2000). }\end{array}$ & $\begin{array}{l}\text { Family } \\
\text { intervention } \\
\text { (MSG; PE) }\end{array}$ & $\begin{array}{l}\text { Family } \\
\text { caregivers } \\
\text { and patients }\end{array}$ & Group & $\begin{array}{l}\text { MSG, PE: } \\
14 \text { sessions, } \\
\text { every 2-3 wks } \\
\text { (2 hrs } \\
\text { duration) }\end{array}$ & $9 \mathrm{mths}$ & Clinical & $\begin{array}{l}\text { MSG: A peer leader } \\
\text { trained by } \\
\text { researchers; } \\
\text { PE: psychiatric nurse } \\
\text { experienced in } \\
\text { psychoeducation and } \\
\text { group therapy or } \\
\text { guest speakers (i.e. } \\
\text { mental health } \\
\text { professionals) }\end{array}$ \\
\hline $\begin{array}{l}\text { Chien, } \\
\text { Chan \& } \\
\text { Thompson } \\
(2006)^{\star}\end{array}$ & China & Majority & Chinese & $\begin{array}{l}\text { Psychoeducation: } \\
\text { Practitioner's guide } \\
\text { to Psychoeducation } \\
\text { \& management } \\
\text { (Anderson et al., } \\
\text { 1986); Mutual } \\
\text { support: support } \\
\text { groups theory \& } \\
\text { practise (Galinsky \& } \\
\text { Schopler, 1994) } \\
\text { Family intervention } \\
\text { for Asian Americans } \\
\text { (Bae \& Kung, 2000) }\end{array}$ & $\begin{array}{l}\text { Family } \\
\text { intervention } \\
(\mathrm{MSG} ; \mathrm{PE})\end{array}$ & $\begin{array}{l}\text { MSG: Family } \\
\text { caregivers; } \\
\text { PE: family } \\
\text { caregivers } \\
\text { and patients }\end{array}$ & Group & $\begin{array}{l}\text { MSG; PE: } \\
12 \text { bi-wkly } \\
\text { sessions } \\
\text { ( } 2 \text { hrs } \\
\text { duration) }\end{array}$ & $6 \mathrm{mths}$ & Clinical & $\begin{array}{l}\text { MSG: family carer } \\
\text { assisted by a group } \\
\text { facilitator (a trained } \\
\text { psychiatric nurse) } \\
\text { PE: two trained } \\
\text { psychiatric nurses }\end{array}$ \\
\hline Chien \& & China & Majority & Chinese & Multifamily groups & Family & Family & Individual & 14 sessions, & $7 \mathrm{mths}$ & Clinical & A case manager who \\
\hline
\end{tabular}




\section{SUPPLEMENTARY}

\section{Lee (2010)}

Chien \&

Lee (2013)

China

Majority

Chinese

Chinese

Meditation-based

stress reduction

program (Kabat-

Zinn et al., 1992);

Practise guidelines

(Lehman \&

Lieberman, 2004)

Chien,
Norman \&
Thompson

China

Majority

Chinese

$(2004)^{*}$

Support group

intervention studies

(Galinsky and
Chien \&

Thompson

(2013)

Chien

Thompson

\& Norman*

(2008b)

\section{China \\ Majority \\ Chinese}

.

for family caregivers

Schopler, 1995;

Meissen and Volk,

1995; Toseland and

Rossiter, 1989)

with the phased

development

reflecting accepted

good practice

(Powell, 1994;

Wilson, 1995).

Modified from

author's previous

work (Chien \&

Chan, 2004; Chien,

Thompson \&

Norman, 2008;

Chien, Chan \&

Thompson, 2006).

sychoeducation

mindfulness

MBPP

Support groups

theory \& practise

(Galinsky \&

Schopler, 1994);

how to work with

self-help group

guidelines (Wilson,

1995) caregivers

and patients

every 2 wks

(2 hrs

duration)

12 sessions

bi-wkly (2 hrs

duration)

4 mths

nical

of formal training

from the

researchers.

NR

\section{Family}

intervention

(FPGP)

\section{Family}

tervention

(MSG)

Family

and patients

Group

Family

caregivers

Group

4 sessions

every 2-3 wks

(2 hrs

duration)

Family

intervention

(MSG)

Family

caregivers

Group

and patients

12 session

(2 hrs

duration)
Family

caregivers
Multifamily groups

(McFarlane, 2002)

Family

intervention

Group

Chien \&
Wong
China Majority Chinese
Chinese
18 sessions
every 2 wks

9 mths

Clinica
3 mths

Clinical

The principal researcher, an experienced

psychiatric nurse and group worker and a peer leader, elected by the group participants

9 mths

Clinical

Trained research

nurse

wo family carers.

One researcher who was a registered

psychiatric nurse and an experienced group worker, acted as a participant and resource person for the group

Psychiatric nurse trained in a three-day 


\section{SUPPLEMENTARY}

(2007)

Gohar et al. Egypt Majority Egyptian

(2013)

Social cognitive

skills training

(Horan, 2011)

Guo et al. China Majority Chinese

(2010)

Majority patient outcomes research team (PORT) manual

(Lehman et al.

2004)

Habib et al. Pakistan Majority Pakistani

(2015)*

Koolaee \&

Etemadi
(2009)

Kopelowicz

et al. (2003)

USA

Minority

Mexican-

American,

other

Central

American,

Cognitive-

behavioural therapy

manual (Kingdon \&

Turkington, 1994)

Psychoeducation

guidelines

(Anderson et

al.,1986; Stengard,

2003); Behavioural

family management

group intervention

adapted from

communication and

problem-solving

skills training

(Falloon,1981)

Social skills training

(Liberman and

Corrigan 1993

Liberman et al

Caribbean

1993)

Kopelowicz

USA
Mexican-

American
Social cognitive
skills training

(SCST)

(FPGP)

duration)

16 sessions, twice per wk

(1 hr duration-

separated by

a break)

Combined

intervention -

family

(CT)

Family

caregivers

and patients

Group

sessions

once per mth.

4 group

treatments on

the same day

(1 hr duration)

Cognitive - family Patients and Individual

(CaCBTp)

family

members

Family

intervention

(BFM; PE)

Mothers of

the patients

Group

16 sessions,

once or twice

per wk

(approx. $1 \mathrm{hr}$

duration)

BFM; PE:

12 sessions

once per wk
Multifamily groups

(McFarlane, 2002);
Family

intervention
(2 hrs

duration)

\section{Skills training - Family}

family
(ST)

Family

members and

Group

patients

4 sessions

per wk. (1 hr

30 mins

duration)

3 mths

Community

(CMHC)

sessions

per mth (1 hr

\section{Clinical}

Family

members and
Group
12 mths
Community (CMHC) workshop that was

held by a family

therapist and the

researchers

Group leader (first author) who received in-person,

supervised training

from the developers

of SCST in the

United States

Therapists, MD or PhD qualified with clinical experience

The first author, a psychologist with a post-graduate

diploma and training in CBTp

A researcher

Trainers (whose disciplines included nursing,psychology, and social work)
Bilingual/ bicultural clinicians. 
Theory of planned

behaviour (Ajzen,

1991)
(MFG-S; MFG-A) patients

uration)

\begin{tabular}{|c|c|c|c|c|}
\hline $\begin{array}{l}\text { Kulhara et } \\
\text { al. (2009) }\end{array}$ & India & Majority & Indian & $\begin{array}{l}\text { Family } \\
\text { psychoeducational } \\
\text { interventions (e.g. } \\
\text { Leff, 2000) }\end{array}$ \\
\hline $\begin{array}{l}\text { Kumar et al. } \\
(2010)\end{array}$ & India & Majority & Indian & $\begin{array}{l}\text { Metacogntitive } \\
\text { training (MCT) } \\
\text { manual (Moritz \& } \\
\text { Woodward, 2007a) }\end{array}$ \\
\hline $\begin{array}{l}\text { Kung et al. } \\
(2012)^{\star \star}\end{array}$ & USA & Minority & Chinese & $\begin{array}{l}\text { Family } \\
\text { psychoeducation } \\
\text { model (Anderson et } \\
\text { al., 1986) \& } \\
\text { Problem-solving } \\
\text { multifamily group } \\
\text { (McFarlane, 2002) }\end{array}$ \\
\hline
\end{tabular}

Lak et al. China Majority Chinese

(2010)

Li \& Arthur

(2005)

UCLA social and

independent living

skills: basic

conversation skills

module (Liberman,

1990)

psychoeducational

interventions (e.g.
Family

intervention

(PE)

1 session per 9 mths
mth $(40-60$
mins.
duration)

$\begin{array}{lll}\begin{array}{l}\text { Cognitive } \\ \text { (HMCT) }\end{array} & \text { Patients } & \text { Group } \\ \begin{array}{l}\text { Family } \\ \text { intervention } \\ \text { (MFPG) }\end{array} & \text { Caregivers } & \text { Group } \\ & & \\ & \end{array}$

$\begin{array}{ll}2 \text { sessions } & 1 \mathrm{mth} \\ \text { per wk. } & \\ \text { ( } 45-60 \text { mins } & \\ \text { duration) } & \\ 12 \text { sessions } & 6 \mathrm{mths} \\ \text { once every } 2 & \\ \text { wks }(2 \text { hrs } & \\ \text { duration) } & \end{array}$

Skills training

(CBCSM;

CBCSM + SGT)

Family

intervention

(FE)
Patients

Group

15 sessions,

duration)

$8 \mathrm{hrs}$ with the

patient and 36 hrs with the psychiatrists,

psychologists, or

social workers with 1

year of experience

conducting family

groups and trained in

the standard MFG

and the MFG-A

manual

Two mental health professionals trained for 2 months by

consultant

psychiatrists, by

didactic lectures and

'hands-on'

experience with

patients and their families

\section{NR}

\section{Clinical NR}

Clinical

One agency

employer with a

master's degree in

counselling

psychology and 4

years of full-time

clinical experience and the $\mathrm{Pl}$ and first author, with a

master's and a

doctorate in social

work and 9 years of

full-time clinical

experience.

Trainer who followed the instructions and guidelines

of the trainer's

manual

First author, a nurse with experience in family intervention 


\section{SUPPLEMENTARY}

(2015)

China

Majority

Chinese

(Kingdon \&

Turkington, 2004)
(CBT)

Patients

Individual

986; Falloon,

1984; Leff, 1985;

Goldstein et al.,

1986; Barrowclough family.

2 hrs per mth

for 3 mths for

patient \&

family

together

12 sessions in $6 \mathrm{mths}$ first 12 wks, 3

booster

sessions in

subsequent

12 wks (40-50

mins duration)

recovery

programme (IMR)

administration's

evidence-based

illness management

and recovery (IMR)

progam

Illness management

and recovery

implementation

resource kit

(Gingerich \&

Mueser, 2010)

Mann \&

China

Majority

Chinese

Delusional

\section{2 sessions \\ per wk ( $1 \mathrm{hr}$ \\ 30 mins \\ duration)}

Patients

Group

verbalization (Alford

et al., 1982);

Cognitive

behavioural therapy

(Garety et al.,1994);

Thought stopping

(Lamontague et al.,

1983); Attentional

control

Illness

management and

recovery

programme (IMR)

Combined

tervention

(SCP)
6 sessions

twice per wk

(1 hr 30 mins

duration

3 wks

Clinical and experienced

registered nurse

research assistants

educated to diploma

or degree leve

Six experienced

therapists

(psychiatrists or

psychologists)

experienced using

psychotherapy;

trained in the

application of

cognitive therapy for

psychosis and had

expertise in the

application of CBT

for psychosis using a

translated Kingdon

and Turkington

manual.

The first author, an assistant professor and affiliated head

nurse

A clinician

NR 


\section{SUPPLEMENTARY}

\begin{tabular}{|c|c|c|c|c|c|c|c|c|c|c|c|}
\hline $\begin{array}{l}\text { Mausbach } \\
\text { et al. } \\
(2008)^{\star}\end{array}$ & USA & Minority & Latino & $\begin{array}{l}\text { Social cognitive } \\
\text { theory (Bandura, } \\
\text { 1989) }\end{array}$ & $\begin{array}{l}\text { Social cognitive } \\
\text { skills training } \\
\text { (PEDAL) }\end{array}$ & Patients & Group & $\begin{array}{l}\text { Sessions } \\
\text { once per wk } \\
\text { (approx. } 2 \text { hrs } \\
\text { duration) }\end{array}$ & $6 \mathrm{mths}$ & $\begin{array}{l}\text { Community } \\
\text { - Board \& } \\
\text { care } \\
\text { faciilities }\end{array}$ & NR \\
\hline $\begin{array}{l}\text { Naeem et } \\
\text { al. }(2015)^{*}\end{array}$ & Pakistan & Majority & Pakistani & $\begin{array}{l}\text { Cognitive- } \\
\text { behavioural therapy } \\
\text { manual (Kingdon \& } \\
\text { Turkington, 1994) }\end{array}$ & $\begin{array}{l}\text { Cognitive - family } \\
\text { (CaCBTp) }\end{array}$ & $\begin{array}{l}\text { Family } \\
\text { members \& } \\
\text { patients }\end{array}$ & Individual & $\begin{array}{l}6 \text { sessions for } \\
\text { the patient } \\
\text { (plus their } \\
\text { carer who } \\
\text { acted as a co- } \\
\text { therapist) plus } \\
\text { one session } \\
\text { for the whole } \\
\text { family. }\end{array}$ & $\begin{array}{l}\text { Approx. } 4 \\
\text { mths }\end{array}$ & Clinical & $\begin{array}{l}\text { Three experienced } \\
\text { psychology } \\
\text { graduates trained by } \\
\text { the first author. }\end{array}$ \\
\hline $\begin{array}{l}\text { Patterson et } \\
\text { al. }(2005)^{\star}\end{array}$ & USA & Minority & Latino & $\begin{array}{l}\text { Social cognitive } \\
\text { theory (Bandura, } \\
\text { 1989) }\end{array}$ & $\begin{array}{l}\text { Social cognitive } \\
\text { skills training } \\
\text { (PEDAL) }\end{array}$ & Patients & Group & $\begin{array}{l}24 \text { semi-wkly, } \\
\text { sessions ( } 2 \\
\text { hrs duration) }\end{array}$ & $6 \mathrm{mths}$ & Clinical & $\begin{array}{l}\text { Bilingual/bicultural } \\
\text { group leader (with } \\
\text { masters or doctorate } \\
\text { level degree) }\end{array}$ \\
\hline $\begin{array}{l}\text { Ran et al. } \\
(2003)\end{array}$ & China & Majority & Chinese & $\begin{array}{l}\text { The } \\
\text { psychoeducational } \\
\text { family approach } \\
\text { (Anderson et } \\
\text { al.1986) \& the } \\
\text { vulnerability-stress } \\
\text { model (Lalonde, } \\
\text { 1995) }\end{array}$ & $\begin{array}{l}\text { Family } \\
\text { intervention } \\
\text { (FIG) }\end{array}$ & $\begin{array}{l}\text { Family \& } \\
\text { Patients } \\
\text { 'encouraged' } \\
\text { to attend }\end{array}$ & $\begin{array}{l}\text { Individual \& } \\
\text { group. Family } \\
\text { education: once } \\
\text { per month. } \\
\text { Multiple family } \\
\text { workshops:once } \\
\text { every } 3 \text { months }\end{array}$ & $\begin{array}{l}\text { Sessions } \\
\text { once per mth } \\
\text { (approx. 1.5-3 } \\
\text { hrs duration) }\end{array}$ & 9 mths & Community & $\begin{array}{l}\text { Trained psychiatrists } \\
\text { and village doctors. } \\
\text { Village doctors did } \\
\text { not get the same } \\
\text { training as } \\
\text { psychiatrists, but } \\
\text { assisted with the } \\
\text { interventions. }\end{array}$ \\
\hline $\begin{array}{l}\text { Razali et al. } \\
(2000)\end{array}$ & Malaysia & Majority & Malaysian & $\begin{array}{l}\text { Behavioural family } \\
\text { therapy (Falloon et } \\
\text { al., 1984) }\end{array}$ & $\begin{array}{l}\text { Family } \\
\text { intervention } \\
\text { (CMFT) }\end{array}$ & $\begin{array}{l}\text { Family } \\
\text { members \& } \\
\text { patients }\end{array}$ & Individual & $\begin{array}{l}30-45 \text { min } \\
\text { sessions. Two } \\
\text { additional } \\
\text { home-visits } \\
\text { every } 6 \text { mths. }\end{array}$ & 12 mths & $\begin{array}{l}\text { Clinical + } \\
\text { community. } \\
\text { (outpatient } \\
\text { hospital and } \\
\text { two } \\
\text { additional } \\
\text { home visits) }\end{array}$ & $\begin{array}{l}\text { Experienced } \\
\text { research } \\
\text { Psychiatrist/first } \\
\text { author with } \\
\text { specialised training }\end{array}$ \\
\hline $\begin{array}{l}\text { Shin \& } \\
\text { Lukens } \\
\text { (2002) }\end{array}$ & USA & Minority & $\begin{array}{l}\text { Korean } \\
\text { American }\end{array}$ & $\begin{array}{l}\text { Psychoeducation } \\
\text { and family therapy } \\
\text { studies (Anderson } \\
\text { et al., 1986; } \\
\text { McFarlane et al., }\end{array}$ & $\begin{array}{l}\text { Family } \\
\text { intervention } \\
\text { (CSPE) }\end{array}$ & $\begin{array}{l}\text { Patients \& } \\
\text { family } \\
\text { members } \\
\text { (were offered } \\
\text { parallel }\end{array}$ & Group & $\begin{array}{l}10 \text { sessions, } \\
\text { once per wk } \\
\text { (90 min } \\
\text { duration) }\end{array}$ & 10 wks & NR & $\begin{array}{l}\text { First author, a } \\
\text { Korean-speaking } \\
\text { psychiatric social } \\
\text { worker }\end{array}$ \\
\hline
\end{tabular}




\section{SUPPLEMENTARY}

\section{China}

So et al.

Majority Chinese

Metacognitive

training ( Moritz \&

Woodward, 2007)

Cognitive

(MCTd)

Patients

Group

4 sessions,

once per wk

(1 hr duration)

$1 \mathrm{mth}$

NR

A clinical

psychologist who

specialises in

psychosis and

received MCT

raining from the

original authors of MCT.

48 sessions

(PSST) $\quad$ family $\quad$ (time limit of 1

12 mths

$\begin{array}{lll}\text { (PSST) } & \text { family } & \text { (time limit of } 1\end{array}$

Group

treatments

(Liberman \&

social and

hr 15 per wk)

modules (Wallace

et al., 1992)

$\begin{array}{llll}\begin{array}{l}\text { Valencia et } \\ \text { al. }(2010)^{*}\end{array} & \text { Mexico } & \text { Majority } & \text { Mexica } \\ & & & \\ \text { Wahass \& } & \text { Saudi } & \text { Majority } & \begin{array}{l}\text { Saudi } \\ \text { Arabian }\end{array} \\ \text { Kent (1997) } & \text { Arabia } & & \text { Arabian }\end{array}$

Psychosocial

treatments

(Liberman \&

Corrigan, (1993) \&

Liberman (2008)

Coping strategy

enhancement (e.g.

Tarrier, 1992);

Focusing strategy

(e.g. Bentall \&

Slade, 1996);

distraction

techniques (e.g.

Nelson et al., 1991)

Wang et al. China Majority Chinese

(2013)

Social Cognition

and Interaction

Training Manual

(Roberts et al.,

2015)

$\begin{array}{lll}\begin{array}{l}\text { Weng et al. China } \\ (2005)\end{array} & \text { Majority Chinese } & \begin{array}{l}\text { Social skills training } \\ \text { for psychiatric } \\ \text { patients (Liberman } \\ \text { et al., 1989) }\end{array}\end{array}$

Social cognitive

skills training

(SCIT)

Combined

intervention

(MPI)

Skills train
(PSST)

et al., 1989)

Skills training

(MRP)

Patients \&

members (for

some

sessions)

Family

Family interventions Family

Two psychologists (postgraduates in clinical psychology)

Xiang et al. China Majority Chinese

40 session

(1 hr 30 mins

duration)

Maximum of

25 sessions,

3 times per

wk. (1 hr

duration)

Group

4 mths

Community

\section{2 mths}

9 wks

.

NR

20 sessions,

once per wk

20 wks

NR

(1)

Group

NR

2 mths

Clinical

Two clinical

psychologists at the master's and doctorate levels

A therapist who has attended several courses and workshops organised in the UK

\section{Six qualified}

psychiatric

counsellors who had been trained by one of SCIT's developers (DR)

NR

Village doctors

(n)




\section{SUPPLEMENTARY}

(1994)

\begin{tabular}{|c|c|c|c|c|}
\hline $\begin{array}{l}\text { Xiong et al. } \\
\text { (1994) }\end{array}$ & China & Majority & Chinese & $\begin{array}{l}\text { Educational } \\
\text { approaches } \\
\text { (Abramowitz \& } \\
\text { Coursey, 1989); } \\
\text { relatives' groups } \\
\text { (Vaughan et al, } \\
\text { 1992); family } \\
\text { therapy (Leff et al, } \\
\text { 1990); behavioural } \\
\text { treatments (Falloon } \\
\text { et al, 1985; Tarrier } \\
\text { et al, 1989); multi- } \\
\text { component } \\
\text { psychosocial } \\
\text { treatment (Leff et al, } \\
\text { 1985). }\end{array}$ \\
\hline
\end{tabular}

\begin{tabular}{|c|c|c|c|c|}
\hline $\begin{array}{l}\text { Zhang \& } \\
\text { Heqin } \\
(1993)^{\star}\end{array}$ & China & Majority & Chinese & $\begin{array}{l}\text { Family } \\
\text { psychoeducation } \\
\text { approaches (e.g. } \\
\text { Brown et al., 1972; } \\
\text { Leff et al. 1982; } \\
\text { Vaughn \& Leff, }\end{array}$ \\
\hline
\end{tabular}

\begin{tabular}{|c|c|c|c|c|}
\hline $\begin{array}{l}\text { Zhang et al. } \\
\text { (1994) }\end{array}$ & China & Majority & Chinese & $\begin{array}{l}\text { Family } \\
\text { psychoeducation } \\
\text { approaches (e.g. } \\
\text { McFarlane, 1982 }\end{array}$ \\
\hline
\end{tabular}

1976)

1972; Leff et al.,

1985; Falloon et al.

intervention

(PFI)

1987)

nt (Leff et al,

1985)

Goldstein, 1984)

\begin{tabular}{|c|c|c|c|c|c|c|}
\hline $\begin{array}{l}\text { Family } \\
\text { intervention } \\
\text { (FIG) }\end{array}$ & $\begin{array}{l}\text { Family } \\
\text { members \& } \\
\text { patients (if } \\
\text { clinical } \\
\text { condition } \\
\text { stable } \\
\text { enough) }\end{array}$ & $\begin{array}{l}\text { Individual and } \\
\text { group }\end{array}$ & $\begin{array}{l}\text { Counselling } \\
\text { sessions, } \\
\text { once per mth } \\
\text { ( } 45 \text { mins } \\
\text { duration); } \\
\text { Family group, } \\
\text { once per mth } \\
\text { (90 mins } \\
\text { duration); } \\
\text { Plus } \\
\text { individual } \\
\text { sessions with } \\
\text { patient, } \\
\text { leaders of } \\
\text { patient work } \\
\text { staff \& family } \\
\text { members }\end{array}$ & $12-24 \mathrm{mths}$ & $\begin{array}{l}\text { Clinical and } \\
\text { community } \\
\text { (clinic }+ \\
\text { home visits) }\end{array}$ & A therapist \\
\hline $\begin{array}{l}\text { Family } \\
\text { intervention } \\
\text { (FE) }\end{array}$ & $\begin{array}{l}\text { Family } \\
\text { members }\end{array}$ & Group & $\begin{array}{l}\text { First } 5 \text { wks: } 4 \\
\text { lectures \& } \\
\text { one group } \\
\text { discussion } \\
\text { (wkly); } \\
\text { 2-6 mths: } 4 \\
\text { lectures and } \\
\text { one group } \\
\text { discussion. } \\
\text { (mthly) } \\
7-12 \text { mths: } 2 \\
\text { lectures and } \\
\text { one group } \\
\text { discussion } \\
\text { (every } 2 \\
\text { mths) }\end{array}$ & $24 \mathrm{mths}$ & NR & $\begin{array}{l}\text { Trained psychiatrists } \\
\text { or nurses }\end{array}$ \\
\hline $\begin{array}{l}\text { Family } \\
\text { intervention } \\
\text { (FIG) }\end{array}$ & $\begin{array}{l}\text { Family } \\
\text { members \& } \\
\text { patients }\end{array}$ & $\begin{array}{l}\text { Individual \& } \\
\text { group }\end{array}$ & $\begin{array}{l}\text { Family group } \\
\text { session: } 3 \\
\text { mths after } \\
\text { discharge; } \\
\text { Families } \\
\text { subsequently } \\
\text { offered } \\
\text { individual or } \\
\text { group }\end{array}$ & 18 mths & $\begin{array}{l}\text { Clinical and } \\
\text { community } \\
\text { (clinic }+ \\
\text { home visits) }\end{array}$ & Counsellors \\
\hline
\end{tabular}




\section{SUPPLEMENTARY}

\begin{tabular}{|c|c|c|c|c|c|c|c|c|c|c|c|}
\hline & & & & & & & & $\begin{array}{l}\text { sessions } \\
\text { every } 3 \text { mths } \\
\text { depending on } \\
\text { whether they } \\
\text { had 'complex } \\
\text { or common } \\
\text { problems' }\end{array}$ & & & \\
\hline $\begin{array}{l}\text { Zhang et al. } \\
(1998)^{\star}\end{array}$ & China & Majority & Chinese & $\begin{array}{l}\text { Family } \\
\text { psychoeducation } \\
\text { approaches (e.g. } \\
\text { Brown et al., 1972; } \\
\text { Leff et al. 1982; } \\
\text { Vaughn \& Leff, } \\
\text { 1976) }\end{array}$ & $\begin{array}{l}\text { Family } \\
\text { intervention } \\
\text { (FE) }\end{array}$ & $\begin{array}{l}\text { Family } \\
\text { members }\end{array}$ & Group & $\begin{array}{l}14 \text { sessions } \\
\text { (1 hr duration) } \\
10 \text { in the first } \\
\text { year, } 4 \text { in the } \\
\text { second year }\end{array}$ & 24 mths & NR & $\begin{array}{l}\text { Trained psychiatrists } \\
\text { or nurses }\end{array}$ \\
\hline $\begin{array}{l}\text { Zimmer el } \\
\text { al. (2007) }\end{array}$ & Brazil & Majority & Brazilian & $\begin{array}{l}\text { Integrated } \\
\text { Psychological } \\
\text { Therapy (Muller et } \\
\text { al., 2005) }\end{array}$ & $\begin{array}{l}\text { Cognitive } \\
\text { (IPT) }\end{array}$ & Patients & Group & $\begin{array}{l}12 \text { sessions, } \\
\text { once per wk } \\
\text { (1 hr duration) }\end{array}$ & $3 \mathrm{mths}$ & NR & $\begin{array}{l}\text { A clinical } \\
\text { psychologist } \\
\text { previously trained by } \\
\text { the IPT author }\end{array}$ \\
\hline
\end{tabular}

Note:

*Studies with the same culturally-adapted intervention(s):

PE + MSG: Chien \& Chan (2004); Chien \& Chan (2013); Chien Chan \& Thompson (2006); Chien, Norman \& Thompson (2004); Chien, Thompson \& Norman (2008)

CaCBTp: Habib et al. (2015) \& Naeem et al. (2015)

IMR: Lin et al. (2013) pilot study \& Lin et al. (2013) RCT

PEDAL: Mausbach et al. (2008) \& Patterson et al. (2005)

PSST: Valencia et al. (2007) \& Valencia et al. (2010)

FE: Zhang \& Heqin (1993) \& Zhang et al. (1998)

** Non-RCT pilot studies: Kung et al. (2012); Lin et al. (2013)

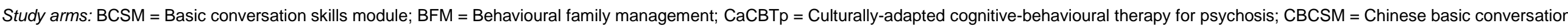

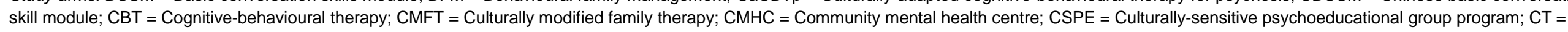

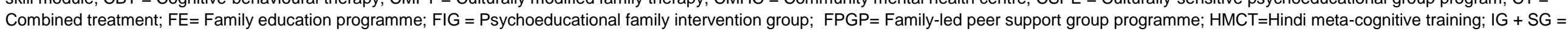

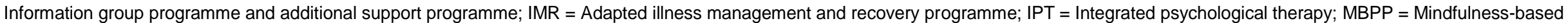

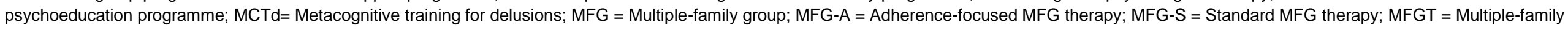

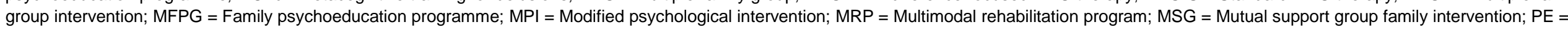

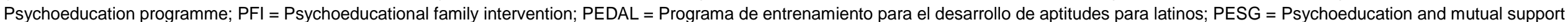

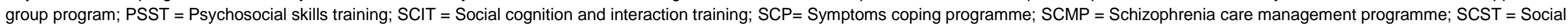
cognitive skills training; SGT = Skills generalisation training; SST = Social skills training; ST = Skills training; TIPSS = Training in interpersonal problem-solving skills 


\section{SUPPLEMENTARY}

\section{Appendix 6}

\section{Table A6: Description and examples of themes of cultural adaptation}

\begin{tabular}{|c|c|c|c|}
\hline Theme & Sub-themes & Description & Examples \\
\hline $\begin{array}{l}\text { Language } \\
\text { Incorporating literal } \\
\text { translation and culturally- } \\
\text { specific forms of expression } \\
\text { and dialect }\end{array}$ & Direct translation & $\begin{array}{l}\text { Translation of materials into } \\
\text { national language or use of } \\
\text { interpreters }\end{array}$ & $\begin{array}{l}\text { 'The plain-language statement and consent form was translated for Vietnamese- speaking consumers } \\
\text { and caregivers.' (Bradley et al., 2006, p.523) } \\
\text { Use of an interpreter: 'Because } 20 \text { of the Vietnamese consumer-caregiver participants ( } 80 \text { percent) } \\
\text { were not fluent in English and an interpreter was required for basic communication, the sample was } \\
\text { considered to have a low level of acculturation.' (Bradley et al., 2006, p.523) } \\
\text { The intervention: 'was translated into Chinese language and validated by the researchers.' (Chien, } \\
2008, \text { p.32) } \\
\text { 'The training sessions were translated and adapted into Arabic by the first author who received in- } \\
\text { person, supervised training from the developers of SCST (WPH, MFG) in the United States.' (Gohar et } \\
\text { al., 2013, p.13) } \\
\text { 'Translated into Persian with a high level of equivalence to the original English version.' (Koolaee \& } \\
\text { Etemadi, 2010, p.5) } \\
\text { The intervention: 'was conducted in elementary school-level Spanish by } 2 \text { clinicians and one of us } \\
\text { (A.K.).' (Kopelowicz et al., 2012, p.267) } \\
\text { 'The original MCT has been translated in Hindi and adapted for Hindi speaking patients at the Central } \\
\text { Institute of Psychiatry, Ranchi.' (Kumar et al., 2010, p.153) } \\
\text { 'All materials were translated into Spanish and back-translated by two different persons of Mexican } \\
\text { origins.' (Patterson et al., 2005, p.925) } \\
\text { 'All written material was provided in both Korean and English, and the oral presentations were in } \\
\text { Korean.' (Shin \& Lukens, 2002, p.1127) } \\
\text { 'The SCIT manual was translated into Chinese by one of the authors (MY) who is a native speaker of } \\
\text { Chinese and has been living in England for } 3 \text { years.' (Wang et al., 2013, p.752) }\end{array}$ \\
\hline & Local dialect & $\begin{array}{l}\text { Incorporating local, dialect, } \\
\text { colloquialisms and idioms }\end{array}$ & $\begin{array}{l}\text { 'For exercises involving written vignettes, some translational adjustments were made to better fit the } \\
\text { local vernacular (e.g., using the term "girlfriend" or "boyfriend" instead of "fiancée")' (Gohar et al., 2013, } \\
\text { p.14) } \\
\text { Adaptations included: 'Integrating culture-specific icons and idioms in the materials.' (Mausbach et al., } \\
2008, \text { p.66) } \\
\text { 'Urdu equivalents of CBT jargons were used in the therapy.' (Naeem et al., 2015, p. 146) } \\
\text { 'Adaptations made to substitute formal wordings on presentations and handouts for more colloquial }\end{array}$ \\
\hline
\end{tabular}




\begin{tabular}{|c|c|c|c|}
\hline & & & $\begin{array}{l}\text { Cantonese words.' (So et al., 2015, p.5) } \\
\text { 'We did not use the term module because this word in Spanish is never used in clinical and therapeutic } \\
\text { environments. As an equivalent of 'modules' we used the term 'treatment areas" (Valencia et al., 2007, } \\
\text { p.1400) }\end{array}$ \\
\hline $\begin{array}{l}\text { Concepts and illness } \\
\text { models } \\
\text { Culturally-appropriate } \\
\text { presentation of concepts, } \\
\text { with consideration of } \\
\text { culture-specific belief } \\
\text { systems, mental health } \\
\text { stigma and levels of } \\
\text { education }\end{array}$ & Explanatory models & $\begin{array}{l}\text { Adapting intervention to work } \\
\text { with culture-specific } \\
\text { explanatory models of mental } \\
\text { illness }\end{array}$ & 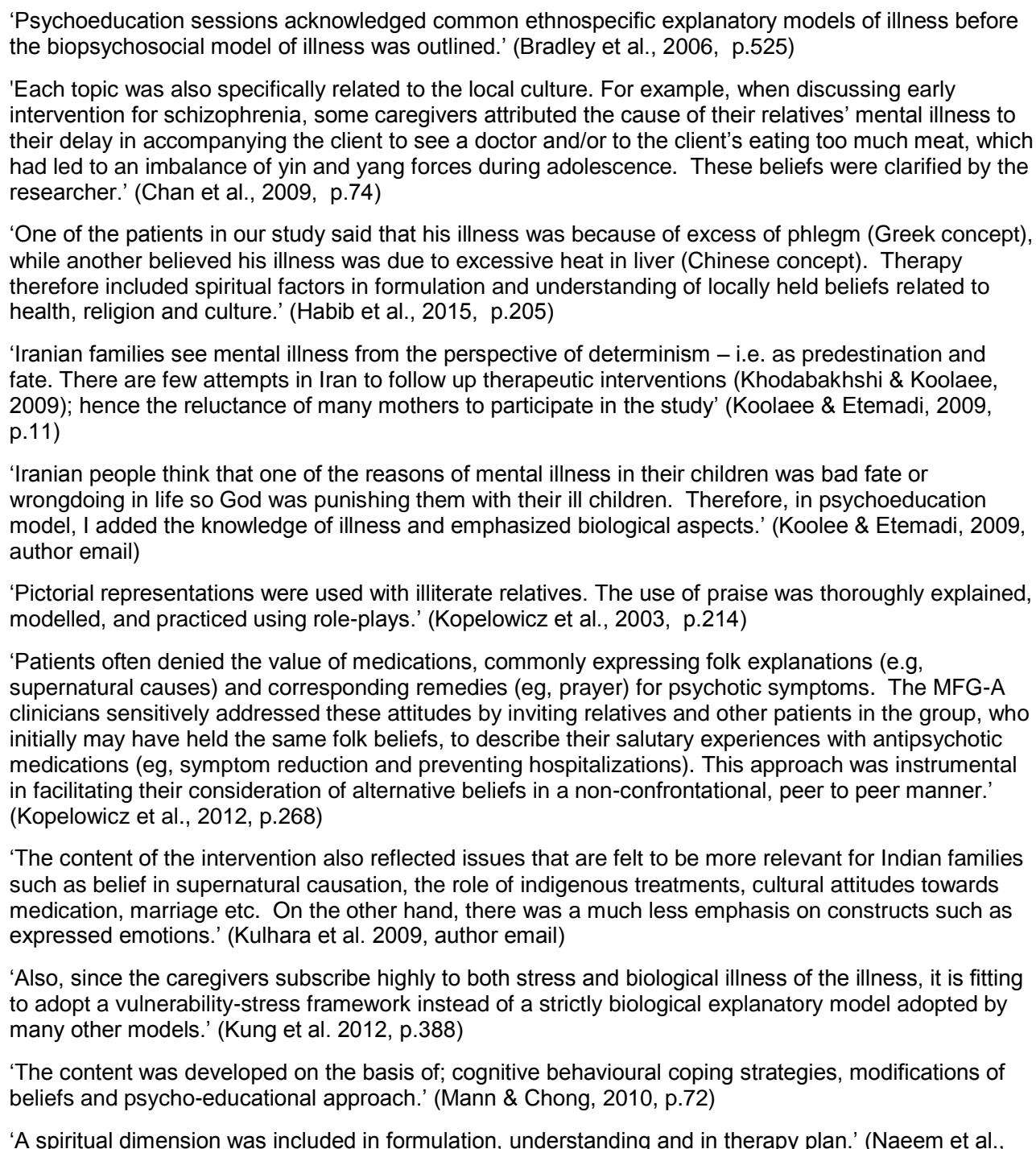 \\
\hline
\end{tabular}




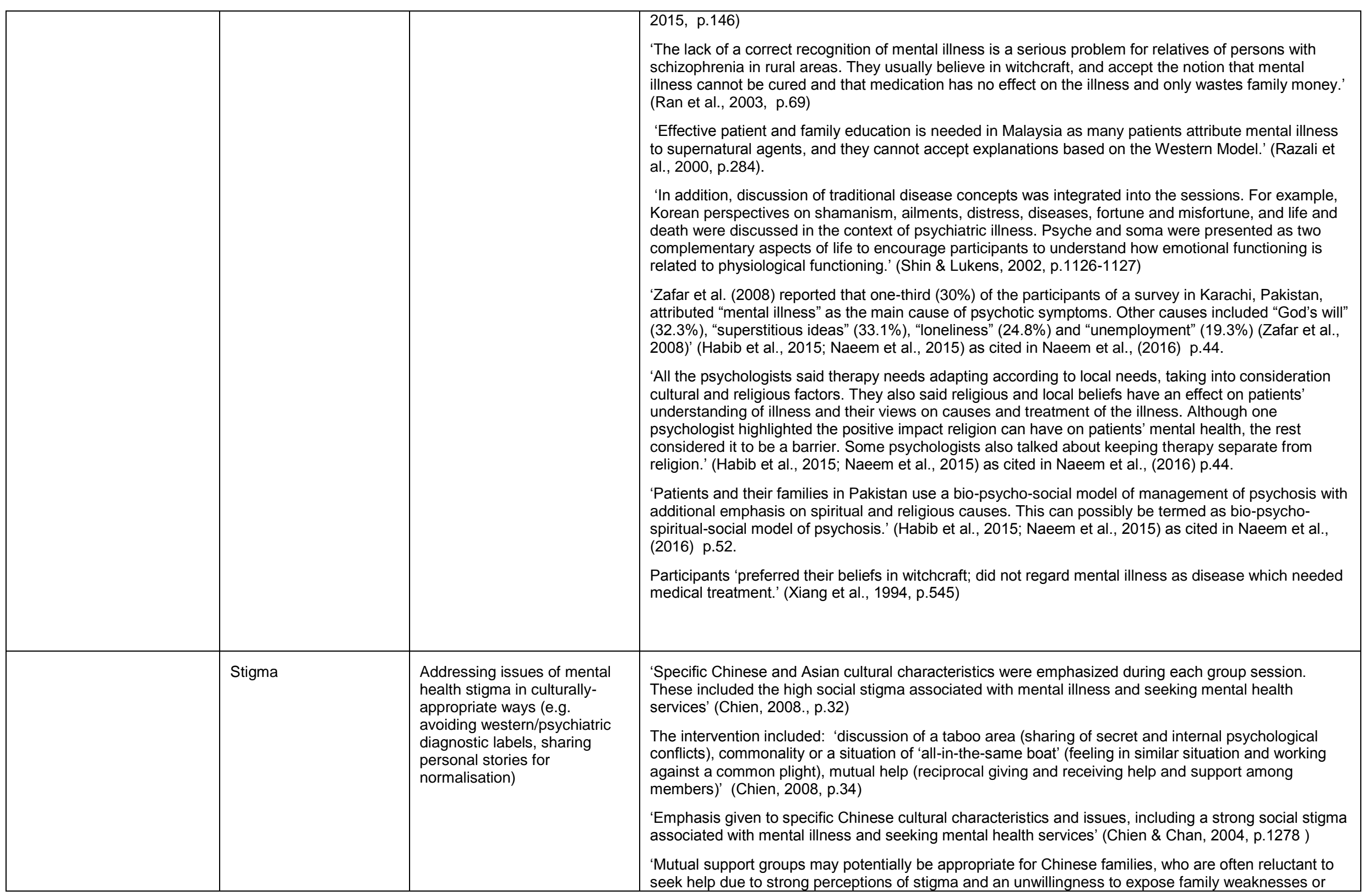




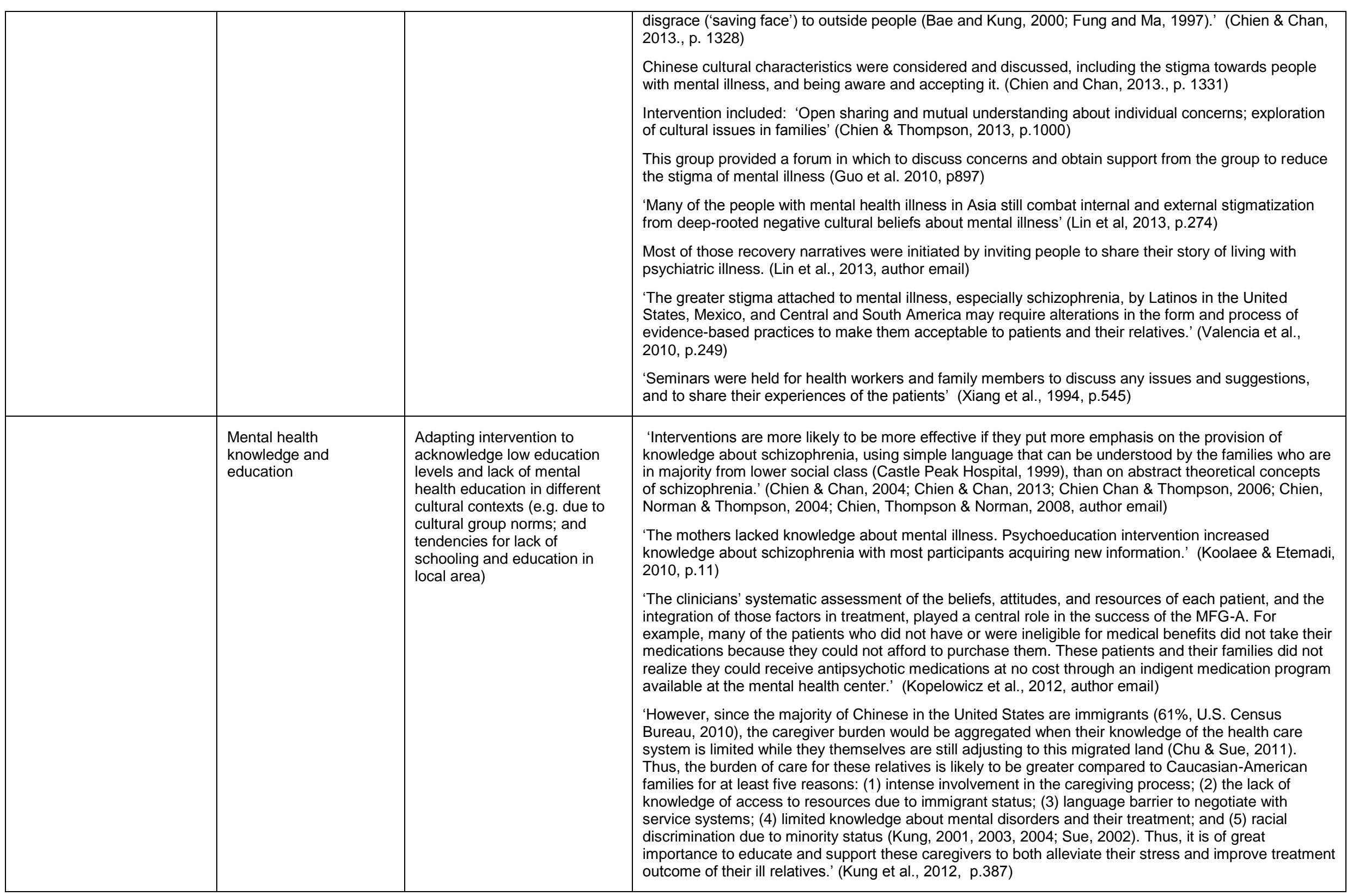




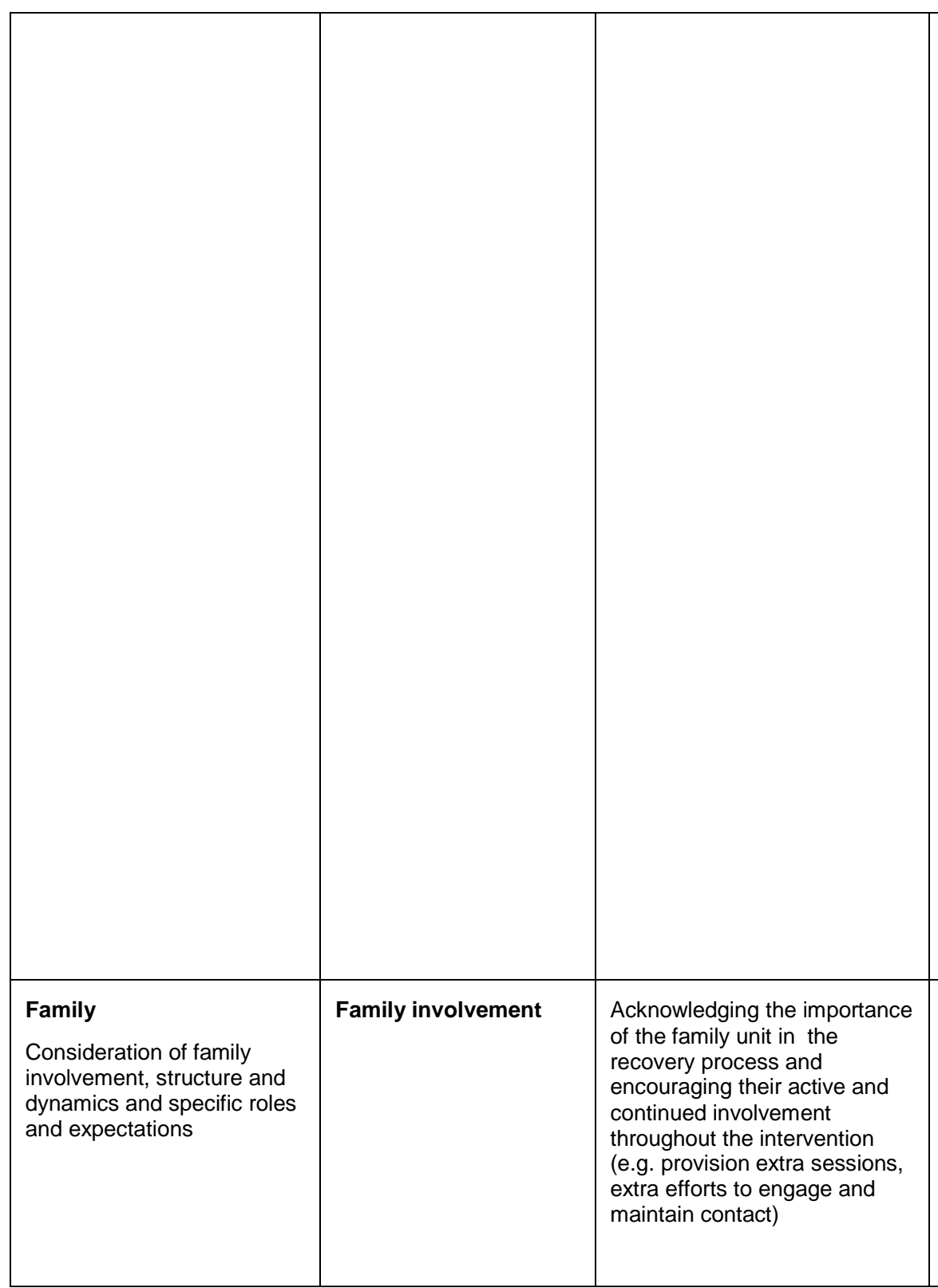

'Psychoeducation materials were given to caregivers throughout the multifamily group sessions instead of a day-long workshop. Due to the lack of knowledge about mental illness and medication, and the lower educational background of many immigrant Chinese-Americans loading them with too much information at the beginning could be overwhelming.' (Kung et al. 2012, p.388)

'Lower educational background of many immigrant Chinese Americans, loading them with too much information at the beginning could be overwhelming, and the retention of the materials limited (Bae \& Kung, 2000).' (Kung et al., 2012, p.388)

'The concept of recovery from psychiatric disorders is still relatively new in Taiwan.' (Lin, Kopelowicz, Chan, \& Hsiung, 2008).' (Lin et al., 2013, author email)

'Written communication was altered to accommodate lower educational backgrounds, and in the transportation module education and materials about public transportation services for disabled persons were provided, due to a lack of knowledge because of previous reliance on family members.' (Patterson et al. 2005, p.525)

'Financial management and written communication were altered to accommodate lower educational backgrounds.' (Patterson et al. 2005, p.925)

The intervention was: 'modified to take account of the characteristics of Chinese rural areas, such as dispersed residences and a generally low level of education.' (Ran et al. 2003., p.70)

'We also employed health education through the village wired radio network.' (Xiang et al., 1994, p.545)

'Compared with Western interventions, in the early stages of family intervention in China much more effort needs to be placed on transforming family members' perception of the role of the physician from that of a pill-provider to that of an informed family advisor.' (Xiong et al., 1994, p.239-240)

'Some families identify social factors as the cause of the problems and try to protect the patient from the influence of these factors by being too controlling and overprotective. Other families do not believe that the patient has a mental illness and think that the bizarre behaviour is under voluntary control; these families tend to be hostile towards the patient.' (Zhang et al., 1994, p.101)

What we did was a reduction in the number of steps, given the complexity of the tasks of the Social Communication subprogram. The stages of this subprogram require a language domain and semantic, grammar, cultural knowledge. In Brazil we have a considerable number of patients with schizophrenia who though illiterate, have little schooling, making it difficult to carrying out steps this subprogram (Zimmer et al. 2007, author email)

'In Asian cultures the family is a crucial social structure, and the burden of illness becomes a joint family obligation, with multiple members engaged in treatment. In contrast, Western cultural values emphasize individualism-for example, protection of the rights of the individual to privacy and confidentiality as well as independent living.' (Bradley et al., 2006, p.529)

'Family joining sessions were conducted informally on an outreach basis in the homes of the Vietnamese families to maximize the likelihood that families would engage with the service and to provide an opportunity to include as many family members as possible.' (Bradley et al., 2006, p.525)

'In Italy, since the deinstitutionalization of the '70s (de Girolamo and Cozza 2000), the key issue in implementing effective treatment systems for severe psychiatric disorders has been balancing community-and hospital-based mental health care (Thornicroft and Tansella 2004). There has been an 


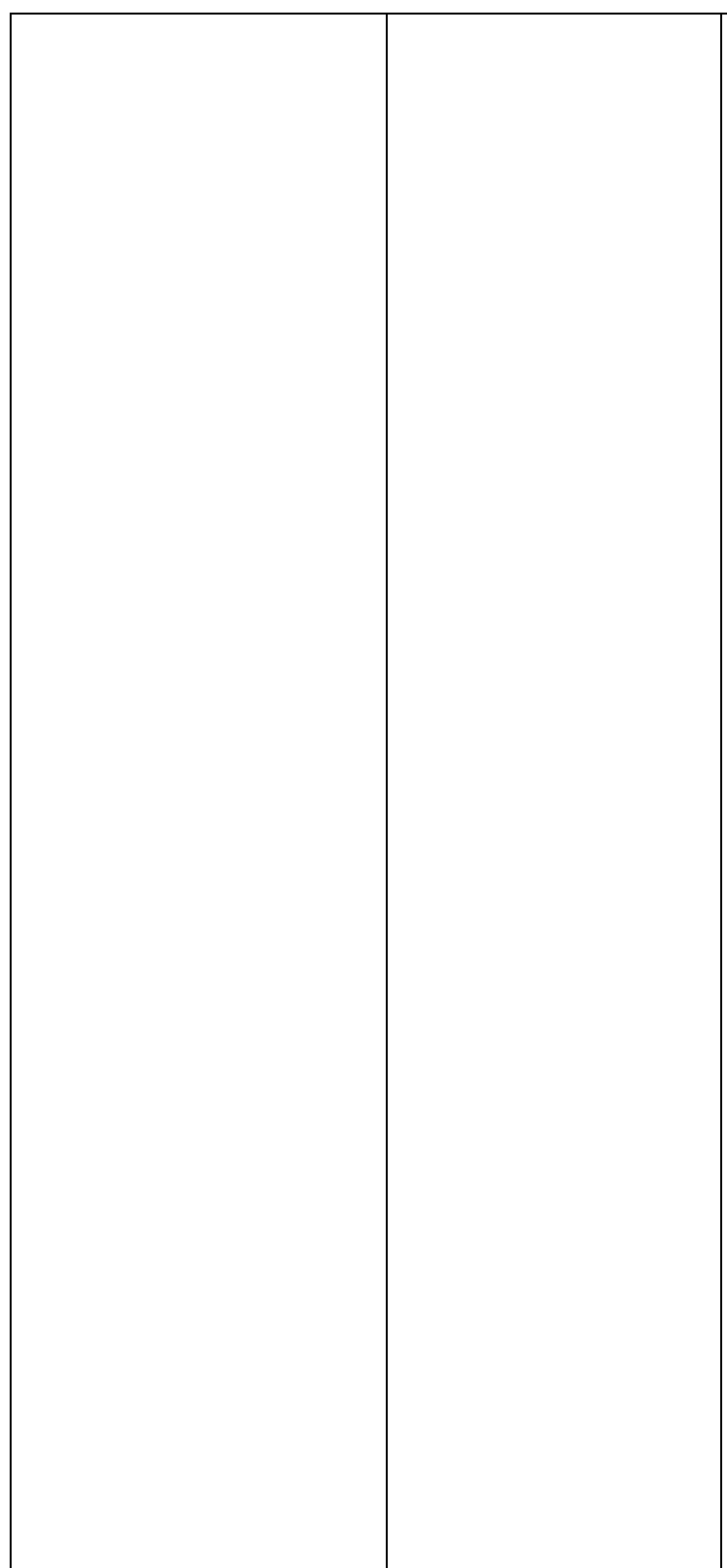

therapeutic potential and the burden they carry.' (Carra et al., 2007, p.24)

'The content of the program was designed according to the preference and perceived needs of patientcaregiver dyads, and the case managers put much emphasis on addressing their cultural issues in family caregiving role.' (Chien \& Lee, 2010, p.318.)

'Family members are actively involved in patient care in Pakistan, and therefore the intervention involved the family members in the treatment plan from the beginning.' (Habib et al., 2015, p.203)

'We chose a family approach because living with supportive relatives increases medication adherence, and interventions that give relatives information about the illness and teach them coping and problemsolving skills reduce relapses and rehospitalizations.' (Kopelowicz et al., 2012, p.265-266)

'This high level of involvement of Indian families in the patients care is partly because of their preference, and partly because of the inadequacy of the mental-health set-up.' (Kulhara et al., 2009, p.473)

'Most families actively want to be involved in all aspects of their relatives wellbeing and problems.' (Kulhara et al., 2009) as cited in Shankar \& Menon, (1993)

'To engage families, especially in the initial stage, in-home single-family sessions were offered in order to involve more relatives.' (Kung et al., 2012, p.388-389)

'Over $80 \%$ of Latinos with schizophrenia live with their famailies (Guarnaccia \& Parra, 1996)... therefore, including families was relevant to the task of maximizing the generalization of skills to the home environment' (Kopelowicz et al., 2003; Kopelowicz et al., 2012) as cited in Lopez \& Kopelowicz (2002) p.15.

'After completing these sessions, skills trainers visited patients and families at their homes to review progress and help solve problems that arose' (Kopelowicz et al., 2003; Kopelowicz et al., 2012) as cited in Lopez \& Kopelowicz (2002) p.15.

'Because of the importance of la familia (the family) in Mexican-American culture, the PEDAL

intervention was adapted to help individuals work with family members for transportation needs rather than help them to utilize public transportation or to travel independently' (Mausbach et al., 2008, p.7273)

'The ultimate social goal of family members of people with schizophrenia in china is to develop a sustainable family-based support system for the dysfunctional individual (Xiong et al., 1994), problem solving and communication skills education was emphasized in this study. In this study the aim was to finish the main content of the education programme in the hospital, and connect with the families after the patients were discharged because few nurses work in community settings and the community mental health services were not sufficiently well developed.' (Li \& Arthur, 2005) as cited in Li (2003) thesis.

'To reinforce the interventions, parallel sessions, also conducted in Korean, were offered to family members of all participants' (Shin \& Lukens., 2002, p.1127)

'Nearly $80 \%$ of Hispanic Americans with Schizophrenia live with their families (Guarnaccia \& Parra, 1996) in Mexico city, it is over 90\%' (Valencia et al., 2003)

'Because of the importance of family cohesion and joint decision making in China, the key family members of patients were involved in some of the training sessions with patients when the topics were 


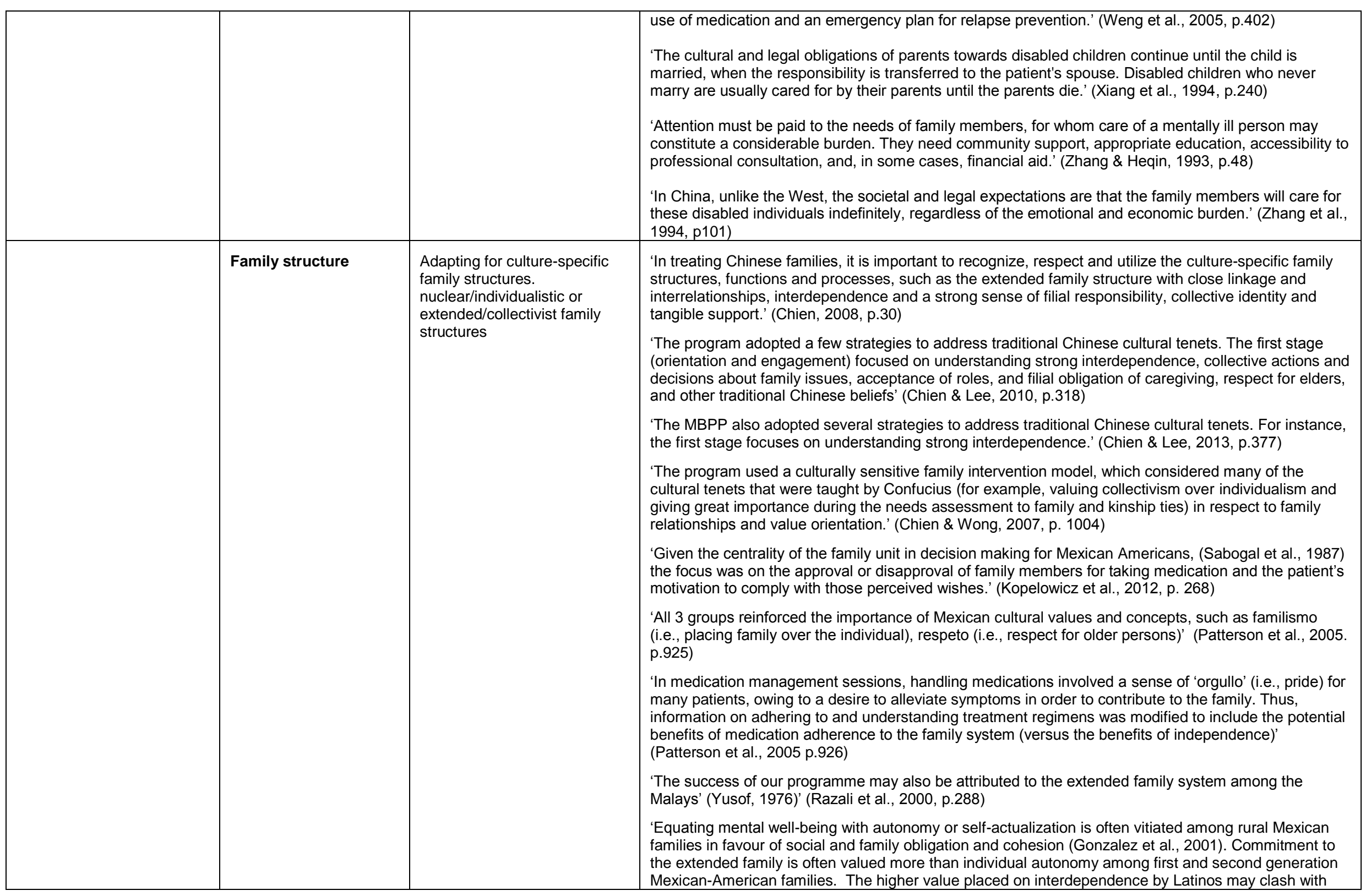




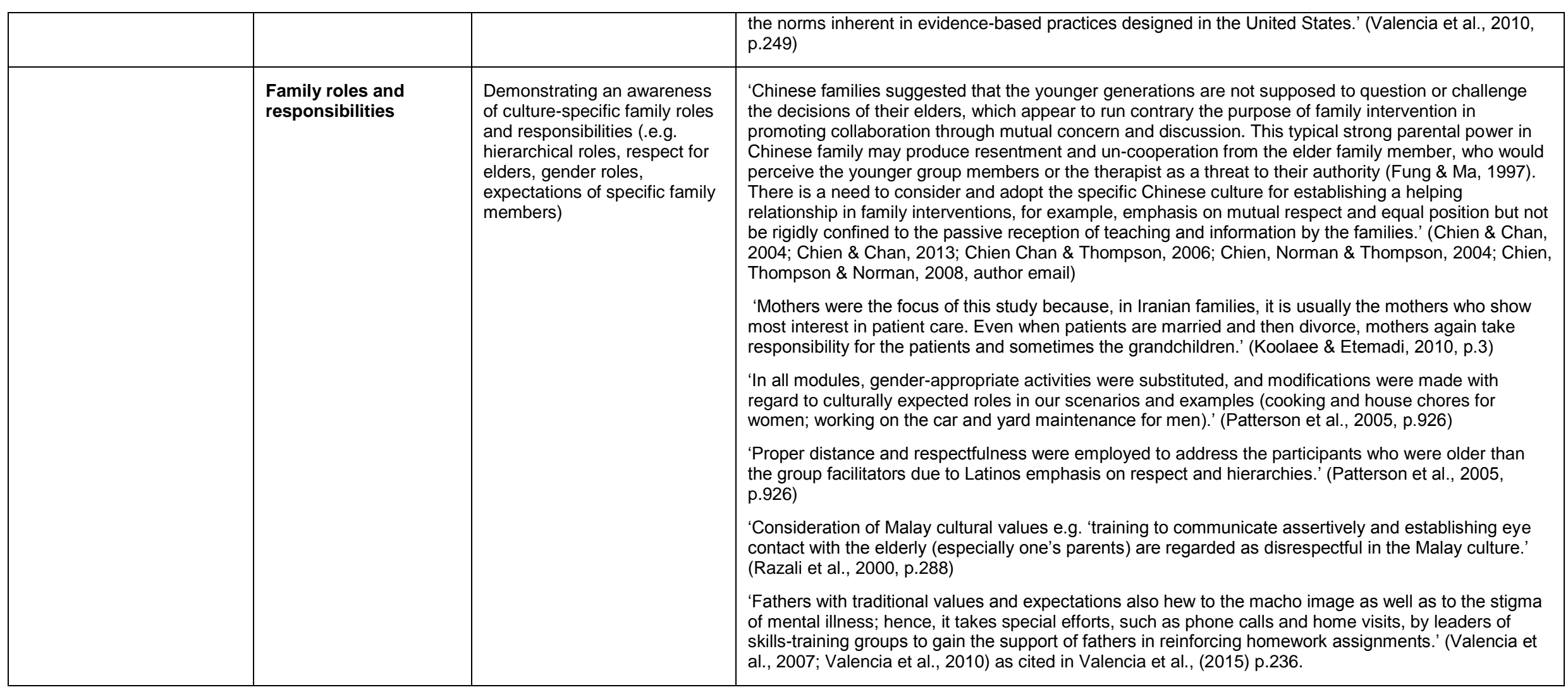




\section{Cultural norms and} practices

Adapting for cultural-specific values and social norms religious/ spiritual beliefs and practices, and incorporating culturallyrelevant social activities
Culturally-specific \begin{tabular}{l|l} 
practices and coping & Adapting intervention to
\end{tabular} $\begin{array}{ll}\text { methods } & \text { accommodate cultural-specific } \\ \text { beliefs, local practices and }\end{array}$ beliefs, local practices and (e.g. spiritual/ religious leaders, spiritual/ religious leaders,
places of worship, experiences of symptoms)
'Traditional alternative healing practices, such as herbal treatments and use of religious leaders, were acknowledged alongside Western approaches.' (Bradley et al., 2006, p. 525)

'It is therefore not surprising that they consult healers from more than one system, for example, faith/spiritual healers and traditional healers as well as consulting doctors. It is also interesting that even those who believed in physical causes contacted different traditional healers, for example faith healers or spiritual healers. Some traditional healers (for example Hakims) use a mixture of Indian, Greek and Chinese medicines.' (Habib et al., 2015, p.204)

'Folk stories and examples from the life of the Prophet Muhammad and Quran were used to clarify issues.' (Habib et al., 2015; Naeem et al., 2015) as cited in Naeem et al., 2010, p.168.

Guidelines for therapists: 'Their belief in supernatural causes of mental illness is not challenged; Emphasis on symptomatic treatment regardless of etiology; Counsellor to have positive attitude toward drugs and have confidence in modern treatment rather than traditional healers.' (Razali et al., 2000, p.285)

'Azhar, Varma and Hakin (1993) examined phenomenological differences in hallucinations between schizophrenic patients in various areas of Malaysia. There were significant differences in the experiences of the Malays of Penang and of Kelantan, indicating that culture affects the phenomenology of hallucinations, even among people of the same race but from different regions.' (Wahass \& Kent, 1997, p.352)

'The content of the voices was influenced by the patients' cultural background. Most Saudi patients reported that their voices involved religious and superstitious themes, while the British patients were most likely to report the giving of instructions.' (Wahass \& Kent, 1997, p.352)

'Considering the important role that religion plays in Islamic cultures, only those strategies that are in harmony with religious beliefs may be accepted by patients and their families. In Islam, for instance, listening to music is not always allowed, so that alternatives may be required for Muslim believers.' (Wahass \& Kent, 1997, p.353)

Designing the intervention to accommodate religious practice: 'While other strategies required modification, being adapted and enhanced to be appropriate for Islamic patients. These coping strategies mostly involved religious beliefs. Under Islamic doctrine, Muslims are required to engage in prayer on five occasions each day, prayers that involve both physical and mental activities. They are also required to perform ablutions before each prayer session, and they must concentrate on their activities. Before, during and after prayer, Muslims read verses of the Quran. If these requirements are fulfilled, believers will be rewarded by god either in this life, in the hereafter, or both.' (Wahass \& Kent, 1997, p.355)

'The therapist encouraged greater engagement with methods such as using prayer, reading the Quran, and regular use of religious practices, as coping methods to control the content and characteristics of the voices. This provided distractions and aided attention switching.' (Wahass \& Kent, 1997, p.355356).

'Coping strategies were based on traditional Islamic beliefs, as held by many people from Islamic backgrounds, which are consistent with the teaching of the Quran. Patients used portable audiocassette players with headphones to listen to a person who was reading the Quran or giving reminders of religious subjects.' (Wahass \& Kent, 1997, p.355-356) 


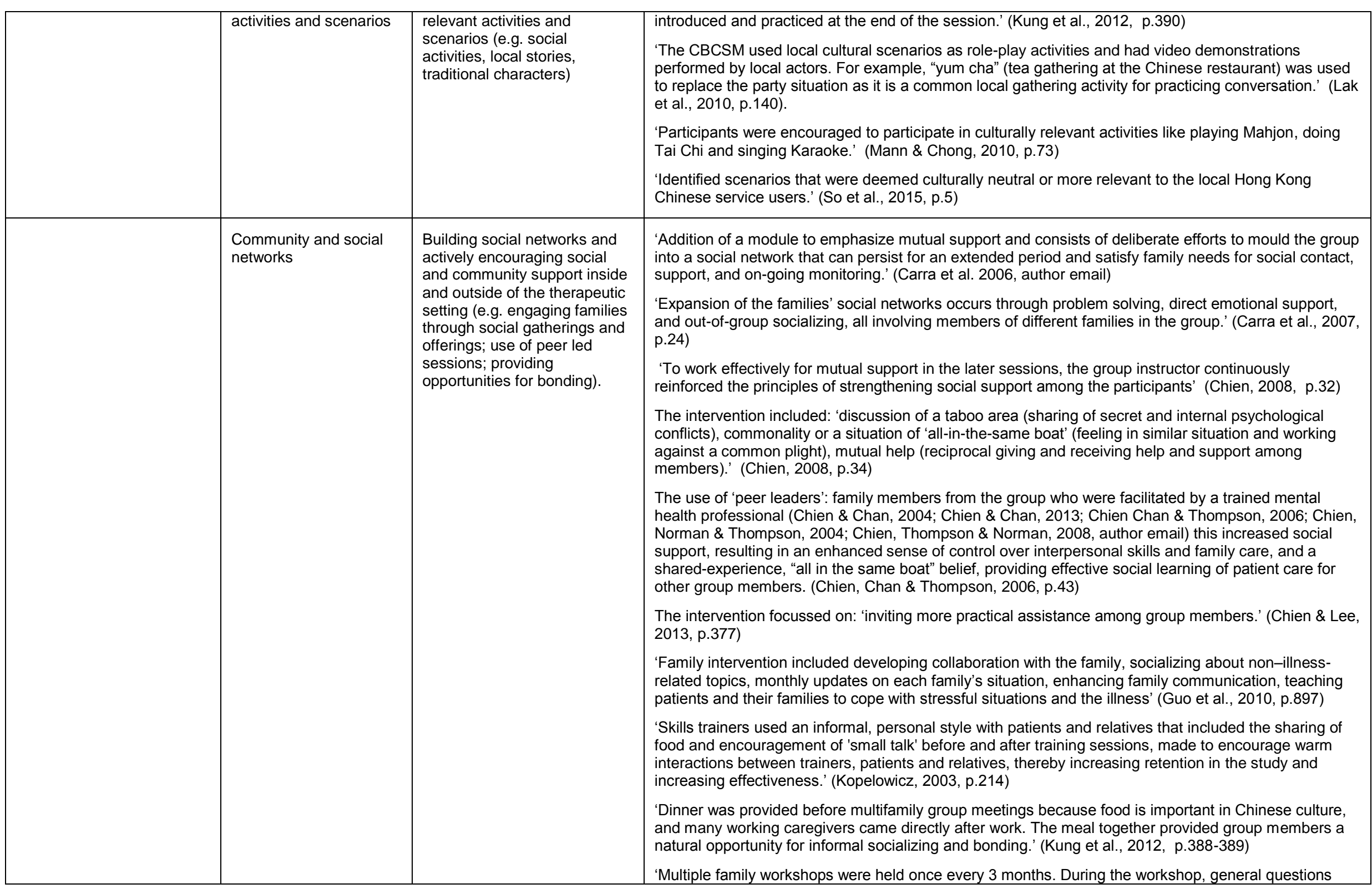




\begin{tabular}{|c|c|c|c|}
\hline & & & $\begin{array}{l}\text { were discussed, and relatives shared the experiences of caring for patients.' (Ran et al., 2003, p.70) } \\
\text { 'Particularly in the earlier sessions, the clinician played an active role in facilitating the group discussion } \\
\text { to encourage and support exchange and sharing.' (Shin \& Lukens, 2002, p.1127) } \\
\text { 'Refreshments were served at every session to encourage attendance' (Shin \& Lukens, 2002, p.1127) }\end{array}$ \\
\hline $\begin{array}{l}\text { Communication Culturally- } \\
\text { specific forms of } \\
\text { communication, problem } \\
\text { solving and learning styles }\end{array}$ & Openness \& disclosure & $\begin{array}{l}\text { Adapting for cultural } \\
\text { differences in open expression } \\
\text { of emotion and/or disclosure of } \\
\text { patient's private information } \\
\text { (e.g. sharing confidential } \\
\text { information, reassurance to } \\
\text { openly discuss problems) }\end{array}$ & $\begin{array}{l}\text { 'Chinese are less likely to express affection to each other through words and touch, than people from } \\
\text { Western countries. They tend to show their concern and feelings for each other through action, for } \\
\text { example, by taking care of actual needs rather than communicating those feelings verbally (Hsu, 1995). } \\
\text { Therefore, it may be difficult to build rapport between the therapist and the family in the traditional } \\
\text { family therapy session (Fung \& Ma, 1997).' (Chien \& Chan, 2004; Chien \& Chan, 2013; Chien Chan \& } \\
\text { Thompson, 2006; Chien, Norman \& Thompson, 2004; Chien, Thompson \& Norman, 2008, author } \\
\text { email) } \\
\text { Group instructor reinforced: 'the principles of strengthening social support among the participants, } \\
\text { including: sharing personal data (ensuring confidentiality and disclosing information with trust.' (Chien } \\
\text { et al., 2008, p.32-33) } \\
\text { 'Chinese families are reluctant to openly disclose their thoughts and feelings in the presence of a } \\
\text { therapist, and that intense emotion should be controlled and hidden, not openly discussed.' (Chien \& } \\
\text { Chan, 2013, p.1328) } \\
\text { Intervention consisted of: 'educational workshop, caregiving role, and therapeutic communication, } \\
\text { learning about home management and effective communication among family members.' (Chien \& Lee, } \\
2010, \text { p.318) } \\
\text { 'They are also assisted in reducing their self-consciousness and need to "save face" (to preserve one's } \\
\text { dignity and avoid any disgrace), reconstruction of their self-image, and improving their insights into } \\
\text { schizophrenia.' (Chien \& Lee, 2013., p.377) } \\
\text { 'Due to the close nature of Mexican families, therapists did not uphold participant confidentiality and } \\
\text { freely shared information about the participants problems and progress with family members.' (Valencia } \\
\text { et al. 2007; Valencia et al., 2010, author email) }\end{array}$ \\
\hline & $\begin{array}{l}\text { Strategies for conflict } \\
\text { resolution and problem } \\
\text { solving }\end{array}$ & $\begin{array}{l}\text { Adapting for cultural-specific } \\
\text { ways of communicating to } \\
\text { resolve problems (e.g. } \\
\text { preferences for direct/ } \\
\text { reparative actions vs. } \\
\text { emotional reassurance; } \\
\text { practical assistance vs. } \\
\text { talking; avoiding confrontation; } \\
\text { assertiveness). }\end{array}$ & $\begin{array}{l}\text { 'There is a need to adapt the family intervention that has originated in the West to take into account } \\
\text { Chinese ways of communication (as characterized by an emphasis on mutual respect and positive } \\
\text { action for family members rather than talking.' (Chien, 2008., p.30) } \\
\text { 'The content of the program was designed according to the preference and perceived needs of patient- } \\
\text { caregiver dyads, and the case managers put much emphasis on addressing their cultural issues in } \\
\text { family caregiving role, effective communication, and resolving conflicts, as well as hands-on practical } \\
\text { experiences.' (Chien \& Lee, 2010, p.318.) } \\
\text { 'Chinese people tend to show their mutual concern and support by seeking to meet each other's actual } \\
\text { needs (Chan et al., 2006) they are reluctant to seek professional help. Therefore, they tend to value } \\
\text { care-giving and therapies which emphasize practical assistance and problem solving rather than } \\
\text { psychological reassurance and opportunities for expression of feelings.' (Chien, Thompson \& Norman, } \\
2008, \text { p.123) } \\
\text { 'Family involvement, differing patterns of communications (for example concept of assertiveness } \\
\text { outside the West) should be important in adapting therapy for local clients in Pakistan.' (Habib et al., }\end{array}$ \\
\hline
\end{tabular}




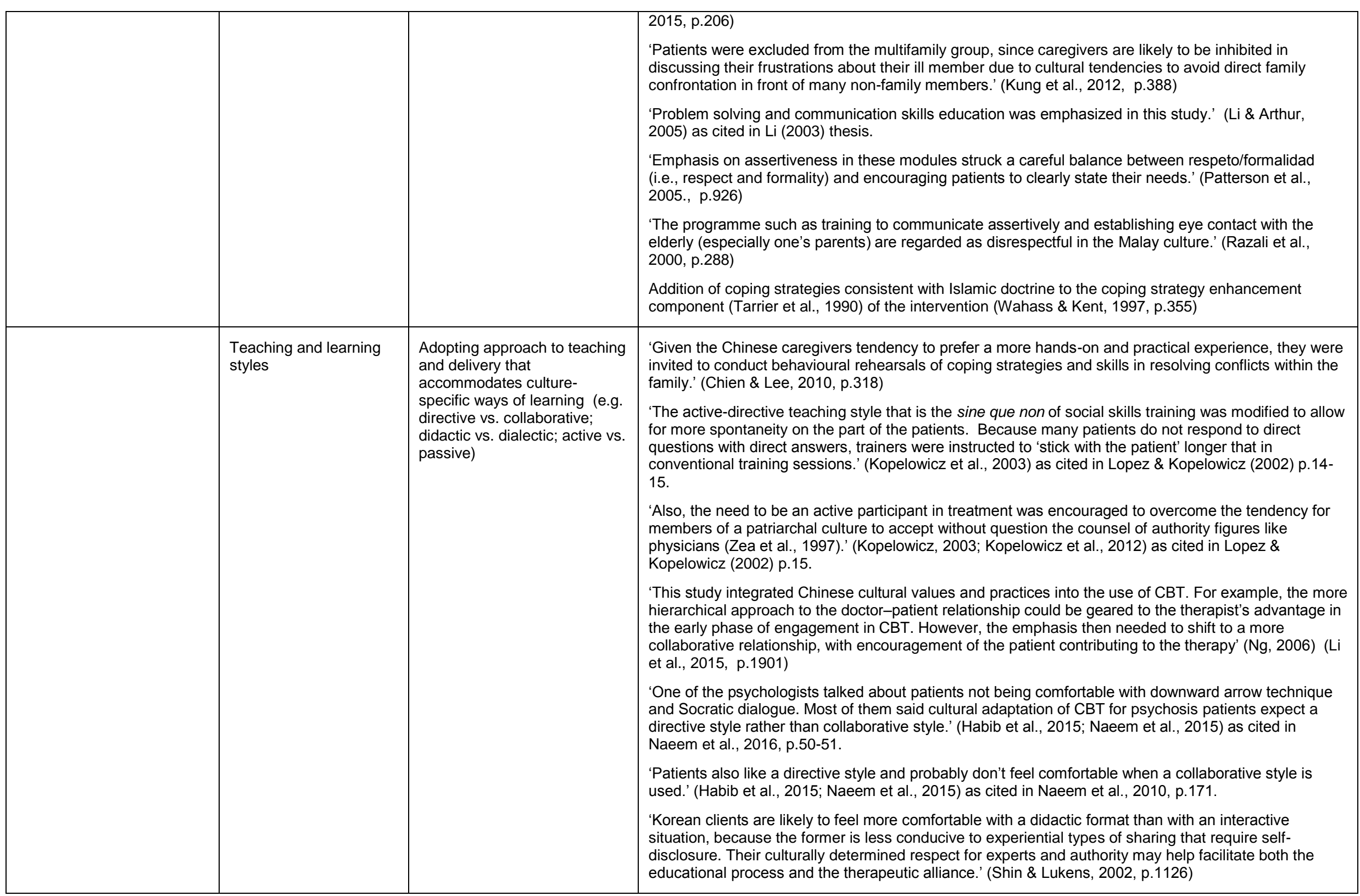




\begin{tabular}{|c|c|c|c|}
\hline & & & $\begin{array}{l}\text { 'Visual aids, including charts and handouts, were used to reinforce the didactic materials.' (Shin \& } \\
\text { Lukens } 2002, \text { p.1127) } \\
\text { 'Family members reported that their ill relatives were uncomfortable with written material, such as } \\
\text { writing exercises during sessions or for homework assignments. Instead, patients considered that } \\
\text { therapy was for talking and learning but not for written activities that reminded them of school activity } \\
\text { that they believed had nothing to do with therapy. Therefore, the skills trainers switched to oral } \\
\text { exercises and assignments, which the patients readily accepted.' (Valencia et al., 2007; Valencia et al., } \\
\text { 2010) as cited in Valencia et al., (2015) p.222. }\end{array}$ \\
\hline \multirow[t]{2}{*}{$\begin{array}{l}\text { Context and delivery } \\
\text { Adapting the delivery of the } \\
\text { intervention to } \\
\text { accommodate, contextual } \\
\text { issues (e.g. lack of } \\
\text { commitment, funding or } \\
\text { resources) to facilitate } \\
\text { feasibility in particular } \\
\text { cultural context }\end{array}$} & Location of intervention & $\begin{array}{l}\text { Delivery of sessions at } \\
\text { accessible and culturally } \\
\text { appropriate location }\end{array}$ & $\begin{array}{l}\text { 'Additional adaptations were made because all of the programs were not delivered within standard } \\
\text { CMHTs but to overcome organisational barriers we provided these outside.' (Carra et al., 2007, author } \\
\text { email) } \\
\text { 'Unlike in the United States, the number of psychiatric beds per capita is on the increase in China, and } \\
\text { community-based services are extremely limited. It is our belief that active promotion of psychiatric } \\
\text { rehabilitation in Chinese psychiatric hospitals at the present time will pave the way for large-scale } \\
\text { implementation of community-based rehabilitation in the future.' (Weng et al., 2005, p.402) } \\
\text { 'In this study the aim was to finish the main content of the education programme in the hospital, and } \\
\text { connect with the families after the patients were discharged because few nurses work in community } \\
\text { settings and the community mental health services were not sufficiently well developed.' (Li \& Arthur, } \\
\text { 2005) as cited in Li (2003) thesis. } \\
\text { 'Providing psychological treatment during the inpatient phase might offer improved opportunities, } \\
\text { especially in a developing country; this is especially important because the distance from health care } \\
\text { facility was reported to be one of the major barriers to receiving therapy regularly (since in Pakistan } \\
\text { most patients and their carers travel long distances to see therapists in psychiatric centres, which are } \\
\text { mainly in large urban areas) (Naeem, Gobbi, Ayub and Kingdon, 2010)' (Habib et al., 2015, p.201) } \\
\text { Delivering the intervention in patients' homes 'as the psychotic patients in the Chinese rural community } \\
\text { reside dispersedly and have different individual problems, they need more specifically tailored } \\
\text { intervention methods conducted in their homes.' (Ran et al., 2003, p.74) } \\
\text { 'The shortage of mental health care in rural China might be tackled by community care.' (Xiang et al., } \\
\text { 1994, p.544) } \\
\text { 'Intervention is provided by hospital-based physicians and nurses since these are the only mental } \\
\text { health professionals available in China.' (Xiong et al., 1994, p.240) }\end{array}$ \\
\hline & $\begin{array}{l}\text { Flexibility in scheduling } \\
\text { sessions }\end{array}$ & $\begin{array}{l}\text { Flexibility in scheduling of } \\
\text { therapy sessions to } \\
\text { accommodate culture (e.g. } \\
\text { frequency, time, intensity) }\end{array}$ & $\begin{array}{l}\text { 'To encourage participation, all of the clients and caregivers were phoned once a week to keep them } \\
\text { engaged during the } 3 \text { months of the PEP. All of the participants were further reminded to attend the } \\
\text { next session } 1 \text { day in advance; repeat sessions were made available to them; and the program was } \\
\text { conducted on the weekends.' (Chan et al., 2009, p.68) } \\
\text { 'We designed this comprehensive psychosocial intervention to be delivered on the same day once a } \\
\text { month mainly owing to the care structure in China, the potential time and cost burden to patients and } \\
\text { their family members, and the feasibility of adoption by other care settings. In China, most patients with } \\
\text { schizophrenia live with their family members because of limited social welfare for severely mentally ill } \\
\text { patients. Many of these family members also work full time, so it is not convenient for them to take time } \\
\text { off every week and bring the patients for therapy. In addition, all our psychosocial interventions were } \\
\text { group based, so having many patients and their family members come in once a week at the same time }\end{array}$ \\
\hline
\end{tabular}




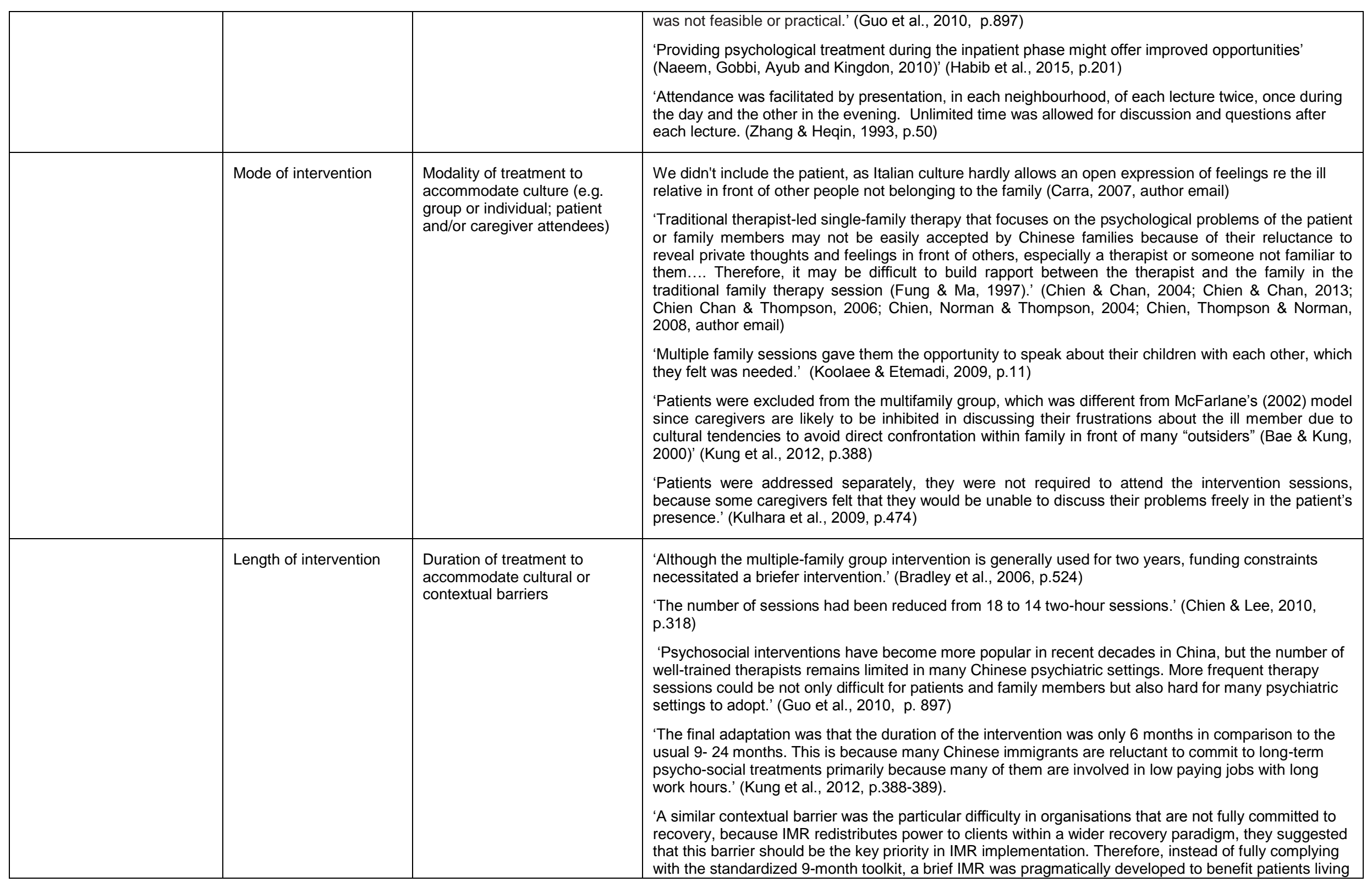




\begin{tabular}{|c|c|c|c|}
\hline & & & $\begin{array}{l}\text { with various degrees of deinstitutionalization and cultural stigmatization.' (Lin et al., 2013, author email) } \\
\text { 'The intervention is ongoing rather than time limited, because stopping it would mean the termination of } \\
\text { any regular follow-up care.' (Xiong et al., 1994, p.240) }\end{array}$ \\
\hline \multirow[t]{2}{*}{$\begin{array}{l}\text { Content } \\
\text { Addition or removal of } \\
\text { specific content }\end{array}$} & $\begin{array}{l}\text { Addition of specific } \\
\text { content }\end{array}$ & $\begin{array}{l}\text { Adding culturally-relevant } \\
\text { content /materials to the } \\
\text { intervention manual }\end{array}$ & 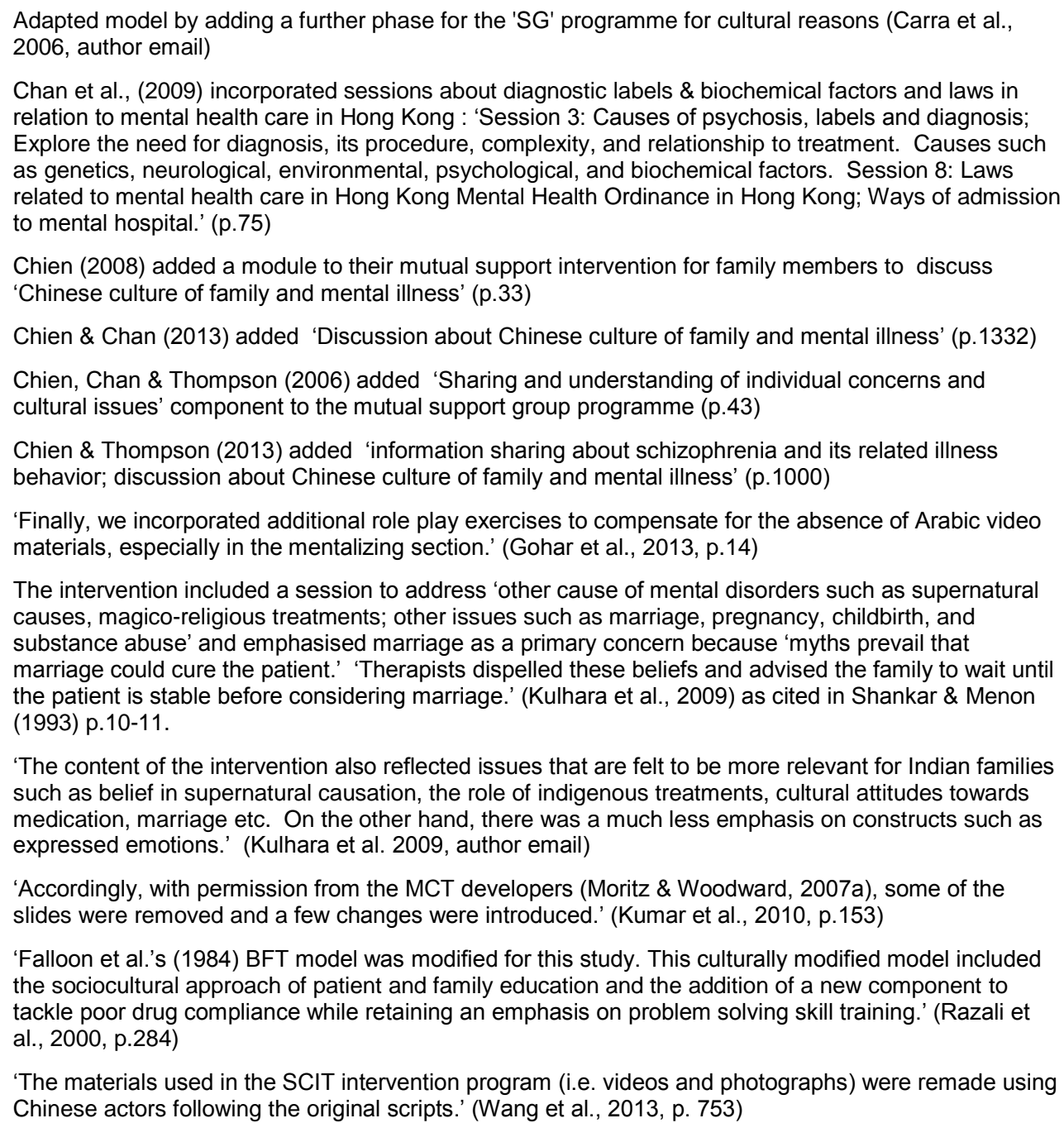 \\
\hline & Removal of specific & $\begin{array}{l}\text { Removing culturally- irrelevant } \\
\text { content/ materials from the }\end{array}$ & 'Modified some written vignettes describing emotions as they relate to pets, as it is not common in \\
\hline
\end{tabular}




\begin{tabular}{|c|c|c|c|}
\hline & content & intervention manual & $\begin{array}{l}\text { Egyptian culture to have a dog or cat in one's home.' (Gohar et al., 2013, p.14) } \\
\text { 'We used most of the picture (e.g., faces, social scenarios), video, and auditory stimuli from the original } \\
\text { version but excluded some that were not well suited to Egyptian culture. For example, we excluded } \\
\text { pictures and videos that depicted unfamiliar recreational activities (e.g., American football or drinking } \\
\text { alcohol beverages)' (Gohar et al., 2013., p.13) } \\
\text { 'We omitted communication skills training as this is the least important among the three core } \\
\text { components of the standard model. This is supported by the finding that generally the carers of Malay } \\
\text { schizophrenic patients could tolerate negative symptoms of schizophrenia (Salleh, 1994)' (Razali et al., } \\
2000, \text { p.284) } \\
\text { 'For example, JTC was illustrated in the original MCT using the conspiracy theory about Paul } \\
\text { McCartney's death. This was substituted by a classic local myth about keeping pregnancy secretive } \\
\text { during the first trimester so as to avoid a miscarriage.' (So et al., 2015, p.5) } \\
\text { 'Only six of the seven learning activities used in the united states were used for Mexican patients. } \\
\text { Video-assisted modelling was not used since skills training technology in Spanish had not been } \\
\text { developed in Mexico. To overcome this obstacle, therapists demonstrated the skills to be learned } \\
\text { during sessions.' (Valencia et al., 2007; Valencia et al., 2010) as cited in Valencia et al. (2015) p.222. } \\
\text { 'Another adaptation was the images used, as in Brazil, they do not have snow, trains and a very small } \\
\text { number of patients can travel by plane.' (Zimmer et al., 2007, author email) }\end{array}$ \\
\hline \multirow[t]{3}{*}{$\begin{array}{l}\text { Therapeutic alliance } \\
\text { Consideration of therapist } \\
\text { qualities/ characteristics, } \\
\text { approach and cultural } \\
\text { competency training to } \\
\text { improve engagement and } \\
\text { alliance }\end{array}$} & $\begin{array}{l}\text { Therapists and client } \\
\text { matched for } \\
\text { characteristics }\end{array}$ & $\begin{array}{l}\text { Therapists matched for } \\
\text { characteristics (e.g. ethnicity, } \\
\text { age, gender, language spoken } \\
\text { etc.) }\end{array}$ & $\begin{array}{l}\text { 'Vietnamese primary therapists were consistent for two intakes of the two cultural groups' (Bradley et } \\
\text { al., 2006, p.524) } \\
\text { 'Cultural adaptations of the program included the use of Vietnamese speaking staff for all aspects of } \\
\text { service provision within the program.' (Bradley et al., 2006, p.525) } \\
\text { Use of a female therapist for Iranian intervention designed for mothers (Koolaee \& Etemadi, 2010, } \\
\text { author email) } \\
\text { 'Additional cultural adaptations included the use of indigenous, bilingual, and bicultural staff of the } \\
\text { community mental health center as skills trainers, the participation of family members (rather than } \\
\text { clinicians) as "generalization aides"' (Kopelowicz et al., 2003, p.214) } \\
\text { 'As the participants in the study spoke either Mandarin or Cantonese, and some were more fluent in } \\
\text { English, trilingual clinicians were sought.' (Kung et al., 2012, p.390) }\end{array}$ \\
\hline & $\begin{array}{l}\text { Therapist 'cultural } \\
\text { competency' training }\end{array}$ & $\begin{array}{l}\text { Therapists received some } \\
\text { form of cultural competency } \\
\text { training and supervision }\end{array}$ & $\begin{array}{l}\text { Trainers 'were all trained to meet cultural sensitivity standards outlined by the State of California and } \\
\text { based on sound empirically-based principles (Rogler et al., 1987; Wallen, 1992). (Kopelowicz et al., } \\
2003 ; \text { Kopelowicz et al., 2012) as cited in Lopez \& Kopelowicz (2002) p.24. } \\
\text { 'Facilitators of these treatments should be educated and familiar with the relevant cultural values of the } \\
\text { consumer.' (Mausbach et al., 2008, p.73) } \\
\text { 'The treating clinicians needed special training because psychiatrists and psychiatric nurses in China } \\
\text { have no experience in the evaluation and management of the family and social problems faced by } \\
\text { mentally ill patients.' (Xiong et al., 1994, p.240) }\end{array}$ \\
\hline & Therapeutic approach & Therapeutic approach adapted & 'Skills trainers used an informal, personal style with patients and relatives that included the sharing of \\
\hline
\end{tabular}




\begin{tabular}{|c|c|c|c|}
\hline & & $\begin{array}{l}\text { to build rapport and trust } \\
\text { between therapist and } \\
\text { patient/family unit (e.g. } \\
\text { informal, warm up activities/ice } \\
\text { breakers, personalised, } \\
\text { general conversation, self- } \\
\text { disclosure) }\end{array}$ & $\begin{array}{l}\text { food and encouragement of 'small talk' before and after training sessions, made to encourage warm } \\
\text { interactions between trainers, patients and relatives, thereby increasing retention in the study and } \\
\text { increasing effectiveness.' (Kopelowicz, 2003, p.214) } \\
\text { 'During the engagement, phase attempts were made to build a positive therapeutic alliance with the } \\
\text { family. Preliminary information (oral printed) about schizophrenia was provided. All this was done in a } \\
\text { no fault atmosphere i.e. without attaching blame to anyone, especially the family.' (Kulhara et al., 2009, } \\
\text { p.474) } \\
\text { 'Intervention focused on establishing trust relationship with patient and family, before identifying their } \\
\text { individual needs.' (Li \& Arthur, 2005, p.340) } \\
\text { Accepting the patient's interpretation of his or her illness to strengthen the therapeutic relationship. } \\
\text { (Razali et al., 2000, p.288) } \\
\text { 'Adaptations to Mexican culture included the therapists beginning the sessions by engaging in platica } \\
\text { (small talk) with the patients which built trust.' (Valencia et al., 2010, p.253) } \\
\text { 'In addition, therapists offered their patients appropriate forms and amounts of self-disclosure from their } \\
\text { own lives which generated a sense of personalismo or a personal orientation to therapeutic } \\
\text { relationships that has been shown to improve the effectiveness of interventions with Latinos (Sue et al., } \\
\text { 1991)' (Valencia et al., 2010, p.253) } \\
\text { 'Each session began with a short warm-up activity (about } 5 \text { min), which was designed to create a more } \\
\text { relaxed atmosphere' (Wang et al, 2013, p.753) }\end{array}$ \\
\hline $\begin{array}{l}\text { Treatment goals } \\
\text { Formulating treatment goals } \\
\text { and encouraging outcomes } \\
\text { that are realistic, culturally } \\
\text { relevant and tailored to the } \\
\text { family }\end{array}$ & $\begin{array}{l}\text { Intervention goals and } \\
\text { expectations of outcome }\end{array}$ & $\begin{array}{l}\text { Ensuring treatment } \\
\text { expectations are realistic and } \\
\text { modifying treatment goals to } \\
\text { ensure culturally relevant (e.g. } \\
\text { colllaborative/shared goals; } \\
\text { cultural values emphasised) }\end{array}$ & $\begin{array}{l}\text { 'Emphasis was given to specific Chinese cultural characteristics and issues, including a strong } \\
\text { tendency to expect immediate and practical help' (Chien \& Chan, 2004 p.1278) } \\
\text { 'Specific Chinese cultural characteristics were emphasised during each group session including the } \\
\text { 'high expectation of immediate and practical help from other family members.' (Meredith et al., 1994; } \\
\text { Bae \& Kung, 2000). (Chien, Chan \& Thompson, 2006, p.44) } \\
\text { 'Emphasis was given to specific Chinese cultural issues, such as their 'high tendency to expect } \\
\text { immediate practical help.' (Chien \& Thompson, 2013, p.999) } \\
\text { 'Given the centrality of the family unit in decision making for Mexican Americans, (Sabogal et al., 1987) } \\
\text { the focus was on the approval or disapproval of family members for taking medication.' (Kopelowicz et } \\
\text { al., 2012, p.268) } \\
\text { Content of sessions included: ' realistic goal setting' (Kulhara et al., 2009, p.474) } \\
\text { 'Basing format, content, and treatment goals on Mexican cultural values such as simpatı'a (the use of } \\
\text { polite social relations (Diaz-Guerraro, 1994; Gloria \& Peregoy, 1996) and personalismo (emphasizing } \\
\text { warm relationships) (Gloria \& Peregoy, 1996; Marin, 1989)' (Mausbach et al., 2008, p.66) } \\
\text { The treatment goals were based on: 'Mexican values and cultural scripts' (Patterson et al, 2005., } \\
\text { p.925) } \\
\text { 'Cultural adaptations were made through the identification of personally relevant goals that often } \\
\text { concerned improving relationships with family members' (Valencia et al., 2007; Valencia et al., 2010) } \\
\text { as cited in Valencia et al., (2015) p.230. }\end{array}$ \\
\hline
\end{tabular}




\begin{tabular}{|c|c|c|c|c|c|c|c|c|c|c|c|}
\hline \multirow[b]{2}{*}{ Study or Subgroup } & \multicolumn{3}{|c|}{ Adapted intervention } & \multicolumn{3}{|c|}{ Control } & \multicolumn{2}{|r|}{ Std. Mean Difference } & \multirow{2}{*}{\multicolumn{3}{|c|}{$\begin{array}{c}\text { Std. Mean Difference } \\
\text { IV, Random, } 95 \% \mathrm{Cl}\end{array}$}} \\
\hline & Mean & SD & Total & Mean & SD & Total & Weight & IN, Random, $95 \% \mathrm{Cl}$ & & & \\
\hline Chien \& Chan 2004 & 10 & 4 & 32 & 10.5 & 4.1 & 31 & $6.6 \%$ & $-0.12[-0.62,0.37]$ & & 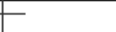 & \\
\hline Chien \& Lee 2010 & 10.1 & 3 & 46 & 10.7 & 4 & 46 & $7.0 \%$ & $-0.17[-0.58,0.24]$ & & & \\
\hline Chien \& Thompson 2013 & 9.9 & 3 & 35 & 9.8 & 3.9 & 35 & $6.7 \%$ & $0.03[-0.44,0.50]$ & & & \\
\hline Chien \& Wong 2007 & 10 & 4 & 42 & 10.5 & 4.1 & 42 & $6.9 \%$ & $-0.12[-0.55,0.31]$ & & & \\
\hline Gohar et al. 2013 & 18.77 & 4.05 & 22 & 17.5 & 3.09 & 20 & $6.0 \%$ & $0.34[-0.27,0.95]$ & & & \\
\hline Habib et al. 2015 & 9.67 & 1.71 & 21 & 21.24 & 5.82 & 21 & $4.9 \%$ & $-2.65[-3.49,-1.80]$ & & & \\
\hline Koolaee \& Etemedi 2009 & 31.44 & 11.16 & 18 & 51.33 & 8.98 & 18 & $5.1 \%$ & $-1.92[-2.72,-1.12]$ & & & \\
\hline Kopelowicz et al. 2003 & 10.9 & 4.9 & 39 & 13.3 & 5.3 & 45 & $6.9 \%$ & $-0.46[-0.90,-0.03]$ & & & \\
\hline Kulhara et al. 2009 & 11.4 & 6.1 & 38 & 13.5 & 5.9 & 38 & $6.8 \%$ & $-0.35[-0.80,0.11]$ & & & \\
\hline Kumar et al. 2010 & 17.87 & 8.58 & 8 & 27.25 & 10.61 & 8 & $4.1 \%$ & $-0.92[-1.97,0.13]$ & & & \\
\hline Li et al. 2015 & 15.23 & 5.78 & 96 & 15.04 & 5.12 & 96 & $7.5 \%$ & $0.03[-0.25,0.32]$ & & & \\
\hline Naeem et al. 2015 & 13.1 & 4.7 & 59 & 16.9 & 5.5 & 57 & $7.1 \%$ & $-0.74[-1.12,-0.36]$ & & & \\
\hline Shin \& Lukens 2002 & 15.08 & 2.75 & 24 & 18.46 & 1.79 & 24 & $5.9 \%$ & $-1.43[-2.07,-0.79]$ & & & \\
\hline So et al. 2015 & 13.23 & 6.5 & 13 & 11.54 & 5.13 & 13 & $5.2 \%$ & $0.28[-0.49,1.05]$ & & & \\
\hline Valencia et al. 2007 & 9.7 & 3.1 & 43 & 13.2 & 4.6 & 39 & $6.8 \%$ & $-0.89[-1.35,-0.44]$ & & & \\
\hline Valencia et al. 2010 & 8.6 & 2.4 & 47 & 11.4 & 4.7 & 36 & $6.8 \%$ & $-0.77[-1.23,-0.32]$ & & & \\
\hline Total $(95 \% \mathrm{Cl})$ & & & 583 & & & 569 & $100.0 \%$ & $-0.56[-0.86,-0.26]$ & & & \\
\hline $\begin{array}{l}\text { Heterogeneity: } \operatorname{Tau}^{2}=0.2 \\
\text { Test for overall effect: } Z=\end{array}$ & $\begin{array}{l}i^{2}=88 \\
(P=0\end{array}$ & df $=$ & $P<0$. & 00001 & -830 & & & & $\begin{array}{cc} & 1 \\
-4 & -2 \\
& \text { Favours [experimental] }\end{array}$ & Favours & $\begin{array}{c}1 \\
2 \\
\text { ontrol] }\end{array}$ \\
\hline
\end{tabular}

A7.1. Forest plot of effect of culturally-adapted psychosocial interventions compared to control on positive symptoms post-treatment

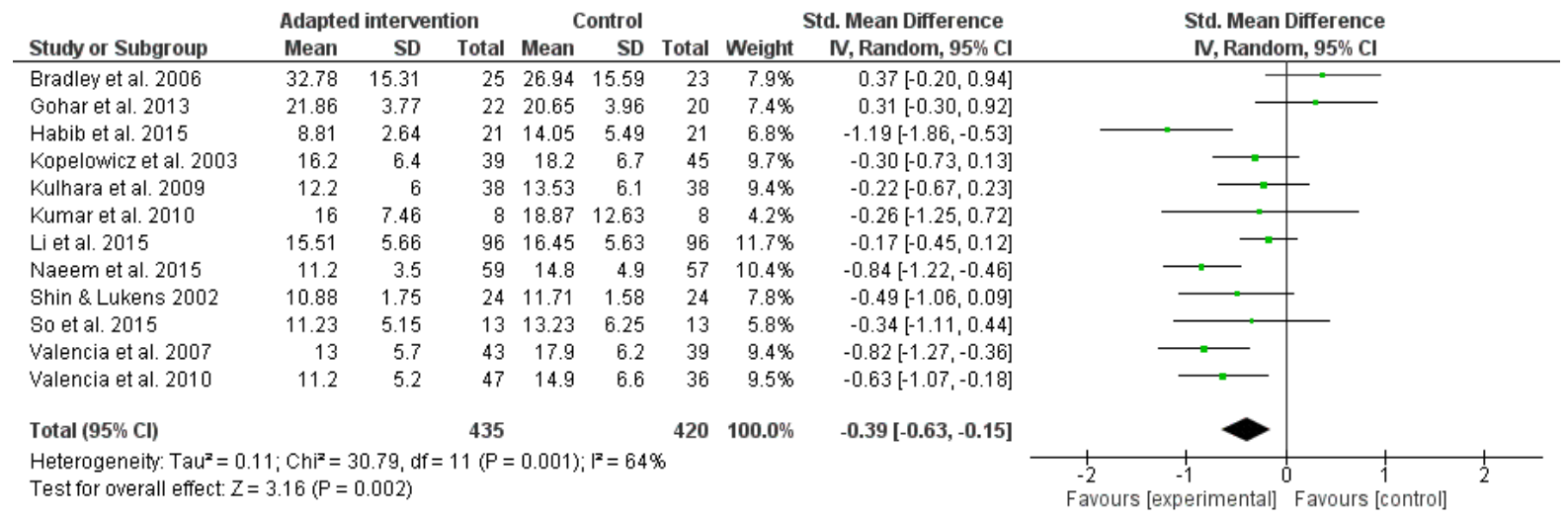

A7.2. Forest plot of effect of culturally-adapted psychosocial interventions compared to control on negative symptoms post-treatment

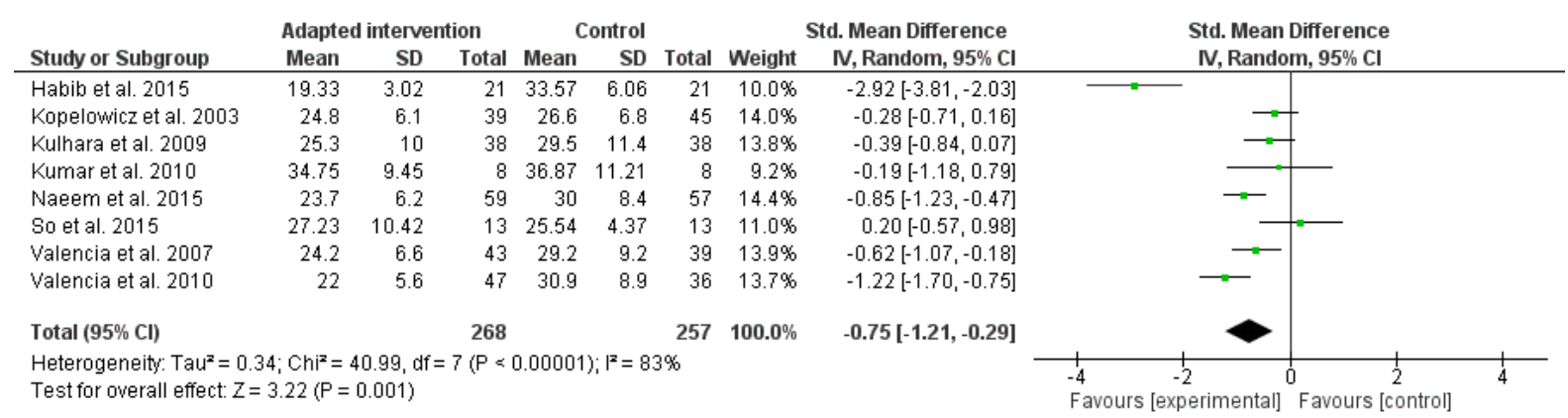

\section{A7.3. Forest plot of effect of culturally-adapted psychosocial interventions compared to control on general symptoms post-treatment}




\section{SUPPLEMENTARY}

\section{Appendix 8}

Table A8.1: Positive symptoms POST-INTERVENTION 0-3 months $(n=16)$

\begin{tabular}{|c|c|c|c|c|c|c|c|c|c|}
\hline \multirow[t]{2}{*}{ Theme } & \multicolumn{3}{|c|}{ SMDs } & \multicolumn{4}{|c|}{ Heterogeneity } & \multirow{2}{*}{$\begin{array}{l}\text { Number of } \\
\text { studies }\end{array}$} & \multirow{2}{*}{$\begin{array}{c}\text { Total sample } \\
\text { size }\end{array}$} \\
\hline & Effect size & $95 \% \mathrm{Cl}$ & $p$ & $\mathrm{Chi}^{2}$ & df & $P(\%)$ & $p$ & & \\
\hline $\begin{array}{l}\text { Intervention type } \\
\text { FI } \\
\text { Cognitive (CBT, MCT) } \\
\text { Skills training (ST, ST + FI) } \\
\text { OR } \\
\text { Skills training (ST, ST + FI) + SCST } \\
\text { Other: (ST, ST + FI, SCST, CBT and MCT) }\end{array}$ & $\begin{array}{l}-0.51 \\
-0.75 \\
-0.70 \\
\\
-0.48 \\
-0.60\end{array}$ & $\begin{array}{l}-0.94 \text { to }-0.08 \\
-1.55 \text { to } 0.05 \\
-0.96 \text { to }-0.45 \\
-0.95 \text { to }-0.01 \\
-1.04 \text { to }-0.16\end{array}$ & $\begin{array}{l}0.02 \\
0.06 \\
0.00001 \\
\\
0.009 \\
0.008\end{array}$ & $\begin{array}{l}30.78 \\
42.99 \\
1.92 \\
\\
11.51 \\
55.61\end{array}$ & $\begin{array}{l}6 \\
4 \\
2 \\
\\
3 \\
8\end{array}$ & $\begin{array}{l}81 \\
91 \\
0 \\
\\
74 \\
86\end{array}$ & $\begin{array}{l}0.0001 \\
0.00001 \\
0.38 \\
\\
\\
0.05 \\
0.008\end{array}$ & $\begin{array}{l}7 \\
5 \\
3 \\
\\
4 \\
9\end{array}$ & $\begin{array}{l}469 \\
392 \\
249 \\
\\
291 \\
683\end{array}$ \\
\hline $\begin{array}{l}\text { Control } \\
\text { Standard care/meds } \\
\text { Enhanced standard care } \\
\text { Active control }\end{array}$ & $\begin{array}{l}-0.84 \\
-0.35 \\
-0.33\end{array}$ & $\begin{array}{l}-1.29 \text { to }-0.38 \\
-0.74 \text { to } 0.03 \\
-1.24 \text { to } 0.58\end{array}$ & $\begin{array}{l}0.0003 \\
0.07 \\
0.47\end{array}$ & $\begin{array}{l}28.15 \\
18.82 \\
19.53\end{array}$ & $\begin{array}{l}6 \\
5 \\
2\end{array}$ & $\begin{array}{l}79 \\
73 \\
90\end{array}$ & $\begin{array}{l}0.0001 \\
0.002 \\
0.0001\end{array}$ & $\begin{array}{l}7 \\
6 \\
3\end{array}$ & $\begin{array}{l}449 \\
421 \\
282\end{array}$ \\
\hline $\begin{array}{l}\text { Region/ Population } \\
\text { Western country/ Minority } \\
\text { Non-western country/Majority }\end{array}$ & $\begin{array}{l}-0.84 \\
-0.45\end{array}$ & $\begin{array}{l}-1.19 \text { to }-0.49 \\
-0.81 \text { to }-0.09\end{array}$ & $\begin{array}{l}0.00001 \\
0.01\end{array}$ & $\begin{array}{l}6.21 \\
66.72 \\
\end{array}$ & $\begin{array}{l}3 \\
11 \\
\end{array}$ & $\begin{array}{l}52 \\
84\end{array}$ & $\begin{array}{l}0.10 \\
0.00001\end{array}$ & $\begin{array}{l}4 \\
12 \\
\end{array}$ & $\begin{array}{l}297 \\
855\end{array}$ \\
\hline $\begin{array}{l}\text { Attendees } \\
\text { Patient only } \\
\text { Family only } \\
\text { Patient \& family }\end{array}$ & $\begin{array}{l}0.05 \\
-1.10 \\
-0.66\end{array}$ & $\begin{array}{l}-0.31 \text { to } 0.41 \\
-2.64 \text { to } 0.44 \\
-1.02 \text { to }-0.31\end{array}$ & $\begin{array}{l}0.78 \\
0.16 \\
\mathbf{0 . 0 0 0 3}\end{array}$ & $\begin{array}{l}4.53 \\
11.15 \\
50.92\end{array}$ & $\begin{array}{l}3 \\
1 \\
9\end{array}$ & $\begin{array}{l}34 \\
91 \\
82\end{array}$ & $\begin{array}{l}0.21 \\
\mathbf{0 . 0 0 0 8} \\
\mathbf{0 . 0 0 0 0 1}\end{array}$ & $\begin{array}{l}4 \\
2 \\
10\end{array}$ & $\begin{array}{l}726 \\
112 \\
764\end{array}$ \\
\hline $\begin{array}{l}\text { Intervention modality } \\
\text { Group } \\
\text { Individual } \\
\text { Both }\end{array}$ & $\begin{array}{l}-0.53 \\
-0.28 \\
-0.83 \\
\end{array}$ & $\begin{array}{l}-0.98 \text { to }-0.07 \\
-0.75 \text { to } 0.19 \\
-1.15 \text { to }-0.51\end{array}$ & $\begin{array}{l}\mathbf{0 . 0 2} \\
0.24 \\
\mathbf{0 . 0 0 0 0 1}\end{array}$ & $\begin{array}{l}56.10 \\
10.46 \\
0.13 \\
\end{array}$ & $\begin{array}{l}9 \\
2 \\
1 \\
\end{array}$ & $\begin{array}{l}84 \\
81 \\
0 \\
\end{array}$ & $\begin{array}{l}\mathbf{0 . 0 0 0 0 1} \\
\mathbf{0 . 0 0 5} \\
0.72 \\
\end{array}$ & $\begin{array}{l}10 \\
3 \\
2\end{array}$ & $\begin{array}{l}539 \\
400 \\
165 \\
\end{array}$ \\
\hline $\begin{array}{l}\text { Attrition } \\
\text { Below } 15 \% \\
\text { Above } 15 \%\end{array}$ & $\begin{array}{l}-0.40 \\
-0.51\end{array}$ & $\begin{array}{l}-0.73 \text { to }-0.07 \\
-0.93 \text { to }-0.08\end{array}$ & $\begin{array}{l}0.02 \\
0.02\end{array}$ & $\begin{array}{l}46.43 \\
8.29\end{array}$ & $\begin{array}{l}9 \\
3\end{array}$ & $\begin{array}{l}81 \\
64\end{array}$ & $\begin{array}{l}0.00001 \\
0.04\end{array}$ & $\begin{array}{l}10 \\
4\end{array}$ & $\begin{array}{l}827 \\
267\end{array}$ \\
\hline $\begin{array}{l}\text { ITT } \\
\text { Yes/ no attrition } \\
\text { No/ 'as treated' }\end{array}$ & $\begin{array}{l}-0.45 \\
-0.70 \\
\end{array}$ & $\begin{array}{l}-0.82 \text { to }-0.07 \\
-1.38 \text { to }-0.03 \\
\end{array}$ & $\begin{array}{l}0.02 \\
0.04\end{array}$ & $\begin{array}{l}59.77 \\
16.11 \\
\end{array}$ & $\begin{array}{l}9 \\
3 \\
\end{array}$ & $\begin{array}{l}85 \\
81 \\
\end{array}$ & $\begin{array}{l}0.00001 \\
0.0001 \\
\end{array}$ & $\begin{array}{l}10 \\
4 \\
\end{array}$ & $\begin{array}{l}825 \\
229 \\
\end{array}$ \\
\hline $\begin{array}{l}\text { Design } \\
\text { RCT } \\
\text { Cluster RCT }\end{array}$ & $\begin{array}{l}-0.65 \\
-0.00 \\
\end{array}$ & $\begin{array}{l}-0.98 \text { to }-0.31 \\
-0.25 \text { to } 0.24\end{array}$ & $\begin{array}{l}\mathbf{0 . 0 0 0 2} \\
0.97 \\
\end{array}$ & $\begin{array}{l}73.04 \\
0.29\end{array}$ & $\begin{array}{l}13 \\
1\end{array}$ & $\begin{array}{l}82 \\
0\end{array}$ & $\begin{array}{l}0.00001 \\
0.59\end{array}$ & $\begin{array}{l}14 \\
2\end{array}$ & $\begin{array}{l}897 \\
255\end{array}$ \\
\hline $\begin{array}{l}\text { Country } \\
\text { Chinese } \\
\text { Not Chinese }\end{array}$ & $\begin{array}{l}-0.07 \\
-0.82\end{array}$ & $\begin{array}{l}-0.29 \text { to } 0.14 \\
-1.23 \text { to }-0.41\end{array}$ & $\begin{array}{l}0.51 \\
0.0001\end{array}$ & $\begin{array}{l}1.28 \\
72.03\end{array}$ & $\begin{array}{l}4 \\
10 \\
\end{array}$ & $\begin{array}{l}0 \\
86\end{array}$ & $\begin{array}{l}0.87 \\
0.00001\end{array}$ & $\begin{array}{l}5 \\
11 \\
\end{array}$ & $\begin{array}{l}335 \\
817\end{array}$ \\
\hline $\begin{array}{l}\text { Pre-treatment differences } \\
\text { No } \\
\text { Yes }\end{array}$ & $\begin{array}{l}-0.65 \\
-0.29\end{array}$ & $\begin{array}{l}-1.01 \text { to }-0.29 \\
-0.64 \text { to } 0.07\end{array}$ & $\begin{array}{l}0.0005 \\
0.11\end{array}$ & $\begin{array}{l}83.72 \\
2.74\end{array}$ & $\begin{array}{l}12 \\
2\end{array}$ & $\begin{array}{l}86 \\
27\end{array}$ & $\begin{array}{l}\mathbf{0 . 0 0 0 0 1} \\
0.25\end{array}$ & $\begin{array}{l}13 \\
3\end{array}$ & $\begin{array}{l}966 \\
186\end{array}$ \\
\hline $\begin{array}{l}\text { Measure } \\
\text { PANSS } \\
\text { BPRS } \\
\end{array}$ & $\begin{array}{l}-0.57 \\
-0.55\end{array}$ & $\begin{array}{l}-0.96 \text { to }-0.18 \\
-1.07 \text { to }-0.03\end{array}$ & $\begin{array}{l}0.005 \\
0.04\end{array}$ & $\begin{array}{l}55.78 \\
30.78\end{array}$ & $\begin{array}{l}9 \\
5\end{array}$ & $\begin{array}{l}84 \\
84\end{array}$ & $\begin{array}{l}0.00001 \\
0.0001\end{array}$ & $\begin{array}{l}10 \\
6\end{array}$ & $\begin{array}{l}759 \\
393\end{array}$ \\
\hline
\end{tabular}

Note:

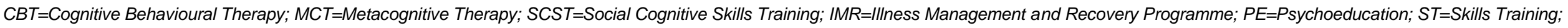
FI=Family Intervention; PANSS=Positive and Negative Symptom Scale; BPRS=Brief Psychiatric Rating Scale; 


\section{SUPPLEMENTARY}

\section{Table A8.2: Negative symptoms POST-INTERVENTION 0-1 months ( $n=12)$}

\begin{tabular}{|c|c|c|c|c|c|c|c|c|c|}
\hline \multirow[t]{2}{*}{ Theme } & \multicolumn{3}{|c|}{ SMDs } & \multicolumn{4}{|c|}{ Heterogeneity } & \multirow{2}{*}{$\begin{array}{c}\text { Number of } \\
\text { studies }\end{array}$} & \multirow{2}{*}{$\begin{array}{c}\text { Total sample } \\
\text { size }\end{array}$} \\
\hline & Effect size & $95 \% \mathrm{Cl}$ & $p$ & $\mathrm{Chi}^{2}$ & df & $R(\%)$ & $p$ & & \\
\hline $\begin{array}{l}\text { Intervention type } \\
\text { FI } \\
\text { Other (CBT, MCT, SCST, ST, ST +FI) } \\
\text { OR } \\
\text { Cognitive (CBT, MCT) } \\
\text { Skills training (ST, ST + FI) } \\
\text { Skills training (ST, ST + FI) + SCST }\end{array}$ & $\begin{array}{l}-0.12 \\
-0.48 \\
-0.57 \\
-0.56 \\
-0.39\end{array}$ & $\begin{array}{l}-0.58 \text { to } 0.35 \\
-0.76 \text { to }-0.21 \\
-1.00 \text { to }-0.14 \\
-0.79 \text { to }-0.33 \\
-0.82 \text { to } 0.03\end{array}$ & $\begin{array}{l}0.62 \\
0.0007 \\
\\
0.01 \\
\mathbf{0 . 0 0 0 0 1} \\
0.07\end{array}$ & $\begin{array}{l}4.61 \\
22.53 \\
\\
12.98 \\
2.76 \\
9.52\end{array}$ & $\begin{array}{l}2 \\
8 \\
4 \\
3 \\
3\end{array}$ & $\begin{array}{l}57 \\
64 \\
\\
69 \\
0 \\
68\end{array}$ & $\begin{array}{l}0.10 \\
0.004 \\
\\
0.01 \\
0.43 \\
0.02\end{array}$ & $\begin{array}{l}3 \\
9 \\
\\
5 \\
3 \\
4\end{array}$ & $\begin{array}{l}172 \\
683 \\
\\
392 \\
297 \\
291\end{array}$ \\
\hline $\begin{array}{l}\text { Control } \\
\text { Standard care/ meds } \\
\text { Enhanced standard care } \\
\text { Active control }\end{array}$ & $\begin{array}{l}-0.66 \\
0.05 \\
-0.14\end{array}$ & $\begin{array}{l}-0.89 \text { to }-0.44 \\
-0.53 \text { to } 0.63 \\
-0.50 \text { to } 0.22\end{array}$ & $\begin{array}{l}\mathbf{0 . 0 0 0 0 1} \\
0.86 \\
0.46\end{array}$ & $\begin{array}{l}7.79 \\
2.52 \\
3.52\end{array}$ & $\begin{array}{l}6 \\
1 \\
2\end{array}$ & $\begin{array}{l}23 \\
60 \\
43\end{array}$ & $\begin{array}{l}0.25 \\
0.11 \\
0.17\end{array}$ & $\begin{array}{l}7 \\
2 \\
3\end{array}$ & $\begin{array}{l}449 \\
124 \\
282\end{array}$ \\
\hline $\begin{array}{l}\text { Region/ Population } \\
\text { Western country/ Minority } \\
\text { Non-western country/Majority }\end{array}$ & $\begin{array}{l}-0.39 \\
-0.39\end{array}$ & $\begin{array}{l}-0.76 \text { to }-0.02 \\
-0.74 \text { to }-0.04\end{array}$ & $\begin{array}{l}0.04 \\
0.03\end{array}$ & $\begin{array}{l}11.51 \\
19.10\end{array}$ & $\begin{array}{l}4 \\
6\end{array}$ & $\begin{array}{l}65 \\
69\end{array}$ & $\begin{array}{l}0.02 \\
0.004\end{array}$ & $\begin{array}{l}5 \\
7\end{array}$ & $\begin{array}{l}345 \\
510\end{array}$ \\
\hline $\begin{array}{l}\text { Attendees } \\
\text { Patient only } \\
\text { Patient \& family }\end{array}$ & $\begin{array}{l}-0.09 \\
-0.54\end{array}$ & $\begin{array}{l}-0.34 \text { to } 0.16 \\
-0.84 \text { to }-0.24\end{array}$ & $\begin{array}{l}0.48 \\
0.0004\end{array}$ & $\begin{array}{l}2.02 \\
18.92\end{array}$ & $\begin{array}{l}2 \\
7\end{array}$ & $\begin{array}{l}1 \\
63\end{array}$ & $\begin{array}{l}0.36 \\
0.008\end{array}$ & $\begin{array}{l}3 \\
8\end{array}$ & $\begin{array}{l}250 \\
529\end{array}$ \\
\hline $\begin{array}{l}\text { Intervention modality } \\
\text { Group } \\
\text { Individual } \\
\text { Both }\end{array}$ & $\begin{array}{l}-0.34 \\
-0.49 \\
-0.38\end{array}$ & $\begin{array}{l}-0.66 \text { to }-0.03 \\
-1.15 \text { to } 0.17 \\
-1.04 \text { to } 0.28\end{array}$ & $\begin{array}{l}\mathbf{0 . 0 3} \\
0.15 \\
0.26\end{array}$ & $\begin{array}{l}11.32 \\
7.81 \\
11.07\end{array}$ & $\begin{array}{l}6 \\
1 \\
2\end{array}$ & $\begin{array}{l}47 \\
87 \\
82\end{array}$ & $\begin{array}{l}0.08 \\
0.005 \\
0.004\end{array}$ & $\begin{array}{l}7 \\
2 \\
3\end{array}$ & $\begin{array}{l}334 \\
308 \\
213\end{array}$ \\
\hline $\begin{array}{l}\text { Attrition } \\
\text { Below } 15 \% \\
\text { Above } 15 \%\end{array}$ & $\begin{array}{l}-0.32 \\
-0.35\end{array}$ & $\begin{array}{l}-0.67 \text { to } 0.02 \\
-0.75 \text { to } 0.05\end{array}$ & $\begin{array}{l}0.07 \\
0.09\end{array}$ & $\begin{array}{l}12.77 \\
11.92\end{array}$ & $\begin{array}{l}4 \\
4\end{array}$ & $\begin{array}{l}69 \\
66\end{array}$ & $\begin{array}{l}0.01 \\
0.02\end{array}$ & $\begin{array}{l}5 \\
5\end{array}$ & $\begin{array}{l}482 \\
315\end{array}$ \\
\hline $\begin{array}{l}\text { ITT } \\
\text { Yes/ no attrition } \\
\text { No/ 'as treated' }\end{array}$ & $\begin{array}{l}-0.42 \\
-0.24\end{array}$ & $\begin{array}{l}-0.79 \text { to }-0.05 \\
-0.66 \text { to } 0.18\end{array}$ & $\begin{array}{l}\mathbf{0 . 0 3} \\
0.26\end{array}$ & $\begin{array}{l}19.20 \\
7.37\end{array}$ & $\begin{array}{l}5 \\
3\end{array}$ & $\begin{array}{l}74 \\
59\end{array}$ & $\begin{array}{l}0.002 \\
0.06\end{array}$ & $\begin{array}{l}6 \\
4\end{array}$ & $\begin{array}{l}516 \\
241\end{array}$ \\
\hline $\begin{array}{l}\text { Country } \\
\text { Chinese } \\
\text { Not Chinese }\end{array}$ & $\begin{array}{l}-0.19 \\
-0.42\end{array}$ & $\begin{array}{l}-0.45 \text { to } 0.08 \\
-0.71 \text { to }-0.14\end{array}$ & $\begin{array}{l}0.17 \\
\mathbf{0 . 0 0 4}\end{array}$ & $\begin{array}{l}0.17 \\
27.56\end{array}$ & $\begin{array}{l}1 \\
9\end{array}$ & $\begin{array}{l}0 \\
67\end{array}$ & $\begin{array}{l}0.68 \\
\mathbf{0 . 0 0 1}\end{array}$ & $\begin{array}{l}2 \\
10\end{array}$ & $\begin{array}{l}218 \\
637\end{array}$ \\
\hline $\begin{array}{l}\text { Pre-treatment differences } \\
\text { No } \\
\text { Yes }\end{array}$ & $\begin{array}{l}-0.43 \\
-0.27\end{array}$ & $\begin{array}{l}-0.75 \text { to }-0.10 \\
-0.56 \text { to } 0.02\end{array}$ & $\begin{array}{l}\mathbf{0 . 0 0 9} \\
0.06\end{array}$ & $\begin{array}{l}29.87 \\
0.10\end{array}$ & $\begin{array}{l}8 \\
2\end{array}$ & $\begin{array}{l}73 \\
0\end{array}$ & $\begin{array}{l}0.0002 \\
0.95\end{array}$ & $\begin{array}{l}9 \\
3\end{array}$ & $\begin{array}{l}669 \\
186\end{array}$ \\
\hline $\begin{array}{l}\text { Measure } \\
\text { PANSS } \\
\text { BPRS + SANS }\end{array}$ & $\begin{array}{l}-0.45 \\
-0.06\end{array}$ & $\begin{array}{l}-0.70 \text { to }-0.20 \\
-0.90 \text { to } 0.79\end{array}$ & $\begin{array}{l}\mathbf{0 . 0 0 4} \\
0.89\end{array}$ & $\begin{array}{l}23.51 \\
4.34\end{array}$ & $\begin{array}{l}9 \\
1\end{array}$ & $\begin{array}{l}62 \\
77\end{array}$ & $\begin{array}{l}0.005 \\
0.04\end{array}$ & $\begin{array}{l}10 \\
2\end{array}$ & $\begin{array}{l}759 \\
96\end{array}$ \\
\hline
\end{tabular}

Note:

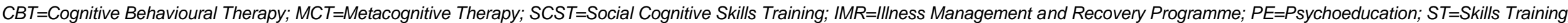

Fl=Family Intervention; PANSS=Positive and Negative Symptom Scale; BPRS=Brief Psychiatric Rating Scale; 


\section{SUPPLEMENTARY}

\section{Table A8.3: General symptoms POST-INTERVENTION 0-1 months $(n=8)$}

\begin{tabular}{|c|c|c|c|c|c|c|c|c|c|}
\hline \multirow[t]{2}{*}{ Theme } & \multicolumn{3}{|c|}{ SMDs } & \multicolumn{4}{|c|}{ Heterogeneity } & \multirow{2}{*}{$\begin{array}{l}\text { Number of } \\
\text { studies }\end{array}$} & \multirow{2}{*}{$\begin{array}{c}\text { Total sample } \\
\text { size }\end{array}$} \\
\hline & Effect size & $95 \% \mathrm{Cl}$ & $p$ & $\mathrm{Chi}^{2}$ & df & $P(\%)$ & $p$ & & \\
\hline $\begin{array}{l}\text { Intervention type } \\
\text { Cognitive (CBT, MCT) } \\
\text { Other (FI, ST, ST + FI) } \\
\text { OR } \\
\text { Skills training }(\mathrm{ST}, \mathrm{ST}+\mathrm{FI})\end{array}$ & $\begin{array}{l}-0.93 \\
-0.62 \\
-0.70\end{array}$ & $\begin{array}{l}-2.05 \text { to } 0.19 \\
-1.02 \text { to } 0.22 \\
-1.23 \text { to }-0.17\end{array}$ & $\begin{array}{l}0.10 \\
0.003 \\
0.01\end{array}$ & $\begin{array}{l}29.55 \\
9.65 \\
8.48\end{array}$ & $\begin{array}{l}3 \\
3 \\
2\end{array}$ & $\begin{array}{l}90 \\
69 \\
76\end{array}$ & $\begin{array}{l}0.00001 \\
0.02 \\
0.01\end{array}$ & $\begin{array}{l}4 \\
4 \\
3\end{array}$ & $\begin{array}{l}200 \\
325 \\
249\end{array}$ \\
\hline $\begin{array}{l}\text { Region/ Population } \\
\text { Western country/ Minority } \\
\text { Non-western country/Majority }\end{array}$ & $\begin{array}{l}-0.70 \\
-0.81 \\
\end{array}$ & $\begin{array}{l}-1.23 \text { to }-0.17 \\
-1.60 \text { to }-0.02 \\
\end{array}$ & $\begin{array}{l}0.01 \\
0.05\end{array}$ & $\begin{array}{l}8.48 \\
32.46 \\
\end{array}$ & $\begin{array}{l}2 \\
4 \\
\end{array}$ & $\begin{array}{l}76 \\
88 \\
\end{array}$ & $\begin{array}{l}0.01 \\
0.00001 \\
\end{array}$ & $\begin{array}{l}3 \\
5 \\
\end{array}$ & $\begin{array}{l}249 \\
276 \\
\end{array}$ \\
\hline $\begin{array}{l}\text { Attendees } \\
\text { Patient only } \\
\text { Patient \& family }\end{array}$ & $\begin{array}{l}0.05 \\
-1.08 \\
\end{array}$ & $\begin{array}{l}-0.55 \text { to } 0.66 \\
-1.68 \text { to }-0.48\end{array}$ & $\begin{array}{l}0.86 \\
\mathbf{0 . 0 0 0 4}\end{array}$ & $\begin{array}{l}0.39 \\
30.86\end{array}$ & $\begin{array}{l}1 \\
4 \\
\end{array}$ & $\begin{array}{l}0 \\
87\end{array}$ & $\begin{array}{l}0.53 \\
\mathbf{0 . 0 0 0 0 1}\end{array}$ & $\begin{array}{l}2 \\
5\end{array}$ & $\begin{array}{l}112 \\
407\end{array}$ \\
\hline $\begin{array}{l}\text { Intervention modality } \\
\text { Group } \\
\text { Individual \& Group }\end{array}$ & $\begin{array}{l}-0.68 \\
-0.92 \\
\end{array}$ & $\begin{array}{l}-1.50 \text { to } 0.14 \\
-1.50 \text { to }-0.33\end{array}$ & $\begin{array}{l}0.11 \\
\mathbf{0 . 0 0 2} \\
\end{array}$ & $\begin{array}{l}33.09 \\
3.26 \\
\end{array}$ & $\begin{array}{l}4 \\
1 \\
\end{array}$ & $\begin{array}{l}88 \\
69 \\
\end{array}$ & $\begin{array}{l}\mathbf{0 . 0 0 0 0 1} \\
0.07\end{array}$ & $\begin{array}{l}5 \\
2\end{array}$ & $\begin{array}{l}224 \\
165\end{array}$ \\
\hline $\begin{array}{l}\text { Attrition } \\
\text { Below } 15 \% \\
\text { Above } 15 \%\end{array}$ & $\begin{array}{l}-0.57 \\
-0.56\end{array}$ & $\begin{array}{l}-1.13 \text { to }-0.01 \\
-1.07 \text { to }-0.05\end{array}$ & $\begin{array}{l}0.05 \\
0.03\end{array}$ & $\begin{array}{l}3.84 \\
11.59\end{array}$ & $\begin{array}{l}1 \\
3\end{array}$ & $\begin{array}{l}74 \\
79\end{array}$ & $\begin{array}{l}0.05 \\
0.009\end{array}$ & $\begin{array}{l}2 \\
4\end{array}$ & $\begin{array}{l}200 \\
267\end{array}$ \\
\hline $\begin{array}{l}\text { ITT } \\
\text { Yes/ no attrition } \\
\text { No/ 'as treated' }\end{array}$ & $\begin{array}{l}-1.30 \\
-0.47 \\
\end{array}$ & $\begin{array}{l}-2.36 \text { to }-0.23 \\
-1.26 \text { to } 0.32 \\
\end{array}$ & $\begin{array}{l}\mathbf{0 . 0 2} \\
0.25\end{array}$ & $\begin{array}{l}24.60 \\
12.95 \\
\end{array}$ & $\begin{array}{l}2 \\
2\end{array}$ & $\begin{array}{l}92 \\
85 \\
\end{array}$ & $\begin{array}{l}0.00001 \\
0.002\end{array}$ & $\begin{array}{l}3 \\
3 \\
\end{array}$ & $\begin{array}{l}234 \\
193 \\
\end{array}$ \\
\hline $\begin{array}{l}\text { Pre-treatment differences } \\
\text { No } \\
\text { Yes }\end{array}$ & $\begin{array}{l}-1.12 \\
-0.25 \\
\end{array}$ & $\begin{array}{l}-1.74 \text { to }-0.50 \\
-0.54 \text { to } 0.04\end{array}$ & $\begin{array}{l}0.0004 \\
0.09 \\
\end{array}$ & $\begin{array}{l}24.52 \\
1.70 \\
\end{array}$ & $\begin{array}{l}4 \\
2 \\
\end{array}$ & $\begin{array}{l}84 \\
0 \\
\end{array}$ & $\begin{array}{l}\mathbf{0 . 0 0 0 1} \\
0.43 \\
\end{array}$ & $\begin{array}{l}5 \\
3 \\
\end{array}$ & $\begin{array}{l}339 \\
186 \\
\end{array}$ \\
\hline
\end{tabular}

Note:

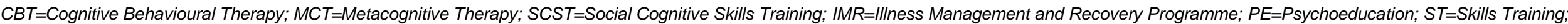
Fl=Family Intervention; PANSS=Positive and Negative Symptom Scale; BPRS=Brief Psychiatric Rating Scale; 
SUPPLEMENTARY

\section{Appendix 9:}

Table A9: Cultural-adaptations emerging from thematic analysis of psychosocial interventions for psychosis (n=46)

\begin{tabular}{|c|c|c|c|c|c|c|c|c|c|}
\hline Author/Theme & Language & $\begin{array}{l}\text { Concepts \& } \\
\text { illness models }\end{array}$ & Family & Cultural norms & Communication & $\begin{array}{l}\text { Context \& } \\
\text { Delivery }\end{array}$ & Content & $\begin{array}{l}\text { Therapeutic } \\
\text { alliance }\end{array}$ & $\begin{array}{l}\text { Treatment } \\
\text { goals }\end{array}$ \\
\hline Bradley et al. (2006) & $\mathrm{x}$ & $x$ & $\mathrm{x}$ & $\mathrm{x}$ & & $x$ & & $x$ & \\
\hline Carrà et al. (2006) & $x$ & & $\mathrm{x}$ & $x$ & & $x$ & $x$ & & \\
\hline Chan et al. (2009) & $\mathrm{x}$ & $x$ & $\mathrm{x}$ & & & $x$ & $\mathrm{x}$ & & \\
\hline Chien (2008) & $\mathrm{x}$ & $x$ & $\mathrm{x}$ & $\mathrm{x}$ & $\mathrm{x}$ & & $\mathrm{x}$ & & $\mathrm{x}$ \\
\hline Chien \& Chan $(2004)^{*}$ & $\mathrm{x}$ & $\mathrm{x}$ & $\mathrm{x}$ & $\mathrm{x}$ & $x$ & & $\mathrm{x}$ & & $\mathrm{x}$ \\
\hline Chien \& Chan $(2013)^{*}$ & $\mathrm{x}$ & $x$ & $\mathrm{x}$ & $\mathrm{x}$ & $x$ & & $\mathrm{x}$ & & $\mathrm{x}$ \\
\hline Chien et al. $(2006)^{*}$ & $\mathrm{x}$ & $x$ & $\mathrm{x}$ & $\mathrm{x}$ & $\mathrm{x}$ & & $x$ & & $x$ \\
\hline Chien \& Lee (2010) & $\mathrm{x}$ & $x$ & $\mathrm{x}$ & & $x$ & $\mathrm{x}$ & & & \\
\hline Chien \& Lee (2013) & $\mathrm{x}$ & & & $\mathrm{x}$ & $\mathrm{x}$ & & & & \\
\hline Chien et al. (2004)* & $x$ & $\mathrm{x}$ & $\mathrm{x}$ & $\mathrm{x}$ & $\mathrm{x}$ & & $x$ & & $\mathrm{x}$ \\
\hline $\begin{array}{l}\text { Chien \& Thompson } \\
\text { (2013) }\end{array}$ & $x$ & $\mathrm{x}$ & $\mathrm{x}$ & $\mathrm{x}$ & $x$ & & $\mathrm{x}$ & & $\mathrm{x}$ \\
\hline Chien et al. (2008)* & $\mathrm{x}$ & $\mathrm{x}$ & $\mathrm{x}$ & $\mathrm{x}$ & $\mathrm{x}$ & & $x$ & & $\mathrm{x}$ \\
\hline Chien \& Wong (2007) & $\mathrm{x}$ & $\mathrm{x}$ & $\mathrm{x}$ & & & & & & 56 \\
\hline
\end{tabular}


SUPPLEMENTARY

\begin{tabular}{|c|c|c|c|c|c|c|c|c|c|}
\hline Author/Theme & Language & $\begin{array}{l}\text { Concepts \& } \\
\text { illness models }\end{array}$ & Family & Cultural norms & Communication & $\begin{array}{l}\text { Context \& } \\
\text { Delivery }\end{array}$ & Content & $\begin{array}{l}\text { Therapeutic } \\
\text { alliance }\end{array}$ & $\begin{array}{l}\text { Treatment } \\
\text { goals }\end{array}$ \\
\hline Gohar et al. (2013) & $x$ & & & $x$ & & $x$ & $x$ & & \\
\hline Guo et al. (2013) & $x$ & $x$ & $x$ & $x$ & & $x$ & & & \\
\hline Habib et al. $(2015)^{*}$ & $x$ & $x$ & $x$ & $x$ & $x$ & $x$ & & & \\
\hline $\begin{array}{l}\text { Koolaee \& Etemadi } \\
(2009)\end{array}$ & $\mathrm{x}$ & $x$ & $\mathrm{x}$ & $\mathrm{x}$ & & $x$ & & $x$ & \\
\hline Kopelowicz et al. (2003) & $x$ & $x$ & $x$ & $x$ & $x$ & & & $x$ & \\
\hline Kopelowicz et al. (2012) & $x$ & $x$ & $x$ & $x$ & $x$ & & & $x$ & $x$ \\
\hline Kulhara et al. (2009) & $x$ & $x$ & $x$ & & & $x$ & $x$ & $x$ & $x$ \\
\hline Kumar et al. (2012) & $x$ & & & & & & $x$ & & \\
\hline Kung et al. $(2012)^{\star *}$ & $x$ & $x$ & $x$ & $x$ & $x$ & $x$ & & $x$ & \\
\hline Lak et al. (2010) & $x$ & & & $x$ & & & & & \\
\hline Li \& Arthur (2005) & $x$ & $x$ & $x$ & & $x$ & $x$ & & & \\
\hline Li et al. (2015) & $x$ & & $x$ & & $x$ & & & & \\
\hline Lin et al. $(2013)^{*}$ & $x$ & $x$ & & & & $x$ & $x$ & & \\
\hline Lin et al. $(2013)^{\star \star}$ & $x$ & $x$ & & & & $x$ & $x$ & & \\
\hline
\end{tabular}


SUPPLEMENTARY

\begin{tabular}{|c|c|c|c|c|c|c|c|c|c|}
\hline Author/Theme & Language & $\begin{array}{l}\text { Concepts \& } \\
\text { illness models }\end{array}$ & Family & Cultural norms & Communication & $\begin{array}{l}\text { Context \& } \\
\text { Delivery }\end{array}$ & Content & $\begin{array}{l}\text { Therapeutic } \\
\text { alliance }\end{array}$ & $\begin{array}{l}\text { Treatment } \\
\text { goals }\end{array}$ \\
\hline Mann \& Chong (2004) & $x$ & $x$ & & $x$ & & & & & \\
\hline Mausbach et al. (2008)* & $x$ & $x$ & $x$ & $x$ & $x$ & & & $x$ & $x$ \\
\hline Naeem et al. $(2015)^{\star}$ & $x$ & $x$ & $x$ & $x$ & $x$ & $x$ & & & \\
\hline Patterson et al. (2005) & $\mathrm{x}$ & $x$ & $x$ & $x$ & $x$ & & & $x$ & $x$ \\
\hline Ran et al. (2003) & $x$ & $x$ & $x$ & & & $x$ & & & \\
\hline Razali et al. (2000) & $x$ & $x$ & $x$ & $x$ & $x$ & & $x$ & $x$ & \\
\hline Shin \& Lukens (2002) & $x$ & $x$ & $x$ & $x$ & $x$ & & & & \\
\hline So et al. (2015) & $x$ & & & $x$ & & & $x$ & & \\
\hline Valencia et al. (2007)* & $x$ & $x$ & $x$ & & $x$ & $x$ & $x$ & $x$ & $x$ \\
\hline Valencia et al. (2010)* & $x$ & $x$ & $x$ & & $x$ & $x$ & $x$ & $x$ & $x$ \\
\hline Wahass \& Kent (1997) & $x$ & & & $x$ & & & & & \\
\hline Wang et al. (2013) & $x$ & & & & & & $x$ & $x$ & \\
\hline Weng et al. (2005) & $x$ & & $x$ & $x$ & & $x$ & & & \\
\hline Xiang et al. (1994) & $x$ & $x$ & $x$ & & & $x$ & & & \\
\hline
\end{tabular}


SUPPLEMENTARY

\begin{tabular}{|c|c|c|c|c|c|c|c|c|c|}
\hline Author/Theme & Language & \begin{tabular}{|l} 
Concepts \& \\
illness models
\end{tabular} & Family & Cultural norms & Communication & $\begin{array}{l}\text { Context \& } \\
\text { Delivery }\end{array}$ & Content & $\begin{array}{l}\text { Therapeutic } \\
\text { alliance }\end{array}$ & $\begin{array}{l}\text { Treatment } \\
\text { goals }\end{array}$ \\
\hline Xiong et al. (1994) & $x$ & $x$ & $x$ & & & $x$ & & $x$ & \\
\hline Zhang \& Heqin $(1993)^{*}$ & $\mathrm{x}$ & $\mathrm{x}$ & $x$ & & & $x$ & & & \\
\hline Zhang et al. (1994) & $\mathrm{x}$ & $\mathrm{x}$ & $x$ & & & & & & \\
\hline Zhang et al. $(1998)^{*}$ & $\mathrm{x}$ & $x$ & $x$ & & & $\mathrm{x}$ & & & \\
\hline Zimmer et al. (2007) & $x$ & $x$ & & & & & $x$ & & \\
\hline Total & 46 & 36 & 35 & 27 & 22 & 22 & 20 & 13 & 13 \\
\hline
\end{tabular}

